\title{
INFINITE DIMENSIONAL LIE GROUPS WITH APPLICATIONS TO MATHEMATICAL PHYSICS
}

\section{RUDOLF SCHMID}

\author{
Communicated by Gregory L. Naber
}

\begin{abstract}
We give a survey of infinite dimensional Lie groups and show some applications and examples in mathematical physics. This includes diffeomorphism groups and their natural subgroups like volume preserving, symplectic and contact transformations, as well as gauge groups, quantomorphisms and loop groups. Various applications include fluid dynamics, Maxwell's equations, plasma physics and BRST symmetries in quantum field theory. We discuss the Lie group structures of pseudodifferential and Fourier integral operators, both on compact and noncompact manifolds and give applications to the $\mathrm{KdV}$ equation and quantization.
\end{abstract}

\section{Introduction}

Lie Groups play an important role in physical systems both as phase spaces and as symmetry groups. Infinite dimensional Lie groups occur in the study of dynamical systems with an infinite number of degrees of freedom such as PDEs and in field theories. For such infinite dimensional dynamical systems diffeomorphism groups and various extensions and variations thereof, such as gauge groups, loop groups and groups of Fourier integral operators occur as symmetry groups and phase spaces. Symmetries are fundamental for Hamiltonian systems. They provide conservation laws (Noether currents) and reduce the number of degrees of freedom, i.e. the dimension of the phase space. Cohomological aspects of Lie groups come into the picture when studying anomalies and BRST symmetries in quantum field theory.

This paper is based on a series of lectures given at the 4th International Conference on Geometry, Integrability and Quantization in Sts Constantine and Elena (Varna) Bulgaria, June 5-16, 2002. We thank the organizers for their good work and their hospitality.

The topics selected for these lectures aim to illustrate some of the ways infinite 
dimensional geometry and global analysis can be used in mathematical problems of physical interest.

1. Infinite dimensional Lie groups

2. Diffeomorphism groups

3. Subgroups of diffeomorphism groups and applications

4. BRST symmetries

5. Lie groups of pseudodifferential- and Fourier integral operators

6. Diffeomorphism groups and FIO for non compact manifolds

7. Applications to fluid dynamics and quantization.

\section{Infinite Dimensional Lie Groups}

In physics Lie groups appear as symmetry groups or configuration spaces of dynamical systems. Some classical, finite dimensional examples are: The linear and angular momentum are related to the groups of translations and rotations; a rigid body's position $f(x, t)$ at time $t$ is determined by $f(x, t)=A(t) \cdot x$, where $A(t) \in S O(3)$ is a proper rotation. The group $S O(3)$ is configuration space and symmetry group. The heavy top has as configuration space also $S O(3)$ and in addition the circle group $S^{1}$ as a symmetry group, which represents rotations about the direction of gravity. "Eliminating" this $S^{1}$ symmetry leaves the Euclidean group $E(3)$ of rigid motions.

Some infinite dimensional examples are: For an incompressible fluid the configuration space is $\operatorname{Diff}_{v o l}(\Omega)$, the volume preserving diffeomorphisms of a region $\Omega$. This is an infinite dimensional "Lie group". For compressible fluids the configuration space is $\operatorname{Diff}(\Omega)$ and as symmetry group acts $\operatorname{Diff}_{\rho}(\Omega)$ the density $\rho$ preserving diffeomorphisms. For plasma physics, which is governed my the MaxwellVlasov equations the configuration space is $\operatorname{Sym}\left(\mathbb{R}^{6}\right)$ the infinite dimensional Lie group of canonical transformations. For the Maxwell's and Yang-Mills equations the group of gauge transformations is an infinite dimensional Lie group which acts a symmetry group. Soliton equations (KdV) can be described as Hamiltonian systems on coadjoint orbits of the Lie group of pseudodifferential operator and the Lie group of Fourier integral operators. 


\subsection{Basic Definitions and Properties}

\subsubsection{Lie Group $\mathcal{G}$}

An infinite dimensional Lie group $\mathcal{G}$ is a group and an infinite dimensional manifold such that the two structures are compatible, in the sense that the group operations multiplication and inversion are smooth maps

$$
\begin{aligned}
& \mu: \mathcal{G} \times \mathcal{G} \rightarrow \mathcal{G}: \mu(g, h)=g h \text { multiplication is } C^{\infty} \\
& \nu: \mathcal{G} \rightarrow \mathcal{G}: \nu(g)=g^{-1} \text { inversion is } C^{\infty} .
\end{aligned}
$$

In finite dimensions, the second condition follows from the implicit function theorem! The group operations satisfy associativity of the product $g(h k)=(g h) k$ and $g e=e g=e, g g^{-1}=g^{-1} g=e$. As a manifold, $\mathcal{G}$ is locally diffeomorphic to an infinite dimensional vector space $V$, which can be a Banach space (with norm $\|$.$\| ), a Hilbert space (with inner product <., .>$ and norm $\|x\|^{2}=\langle x, x>$ ), or a Frechet space (with metric $d(.,$.$) but no norm). Correspondingly we call$ these Banach Lie groups, Hilbert Lie groups or Frechet Lie groups. If $\mathcal{G}$ is locally diffeomorphic to $\mathbb{R}^{n}, n<\infty$, then $\mathcal{G}$ is a finite dimensional Lie group.

\subsubsection{The Differentiable Structure of $\mathcal{G}$}

For $g \in \mathcal{G}$ consider left and right translations, $L_{g}: \mathcal{G} \rightarrow \mathcal{G} ; L_{g}(h)=g h$ and $R_{g}: \mathcal{G} \rightarrow \mathcal{G} ; R_{g}(h)=h g$. These are diffeomorphisms with inverses given by $\left(L_{g}\right)^{-1}=L_{g^{-1}}$ and $\left(R_{g}\right)^{-1}=R_{g^{-1}}$. Let $(U, \phi)$ be a chart at the identity $e \in \mathcal{G}$ and define a chart $\left(U_{g}, \phi_{g}\right)$ at $g \in \mathcal{G}$ by $U_{g}=L_{g}(U)=\left\{L_{g} h ; h \in U\right\}$ and $\phi_{g}=\phi \circ L_{g^{-1}}: U_{g} \rightarrow V, h \mapsto \phi\left(g^{-1} h\right)$. So a single chart at the identity defines the differentiable structure of $\mathcal{G}$ with smooth transition maps (change of coordinates) given by $\phi_{g} \circ \phi_{h}^{-1}=\phi \circ L_{g^{-1} h} \circ \phi^{-1}$.

\subsubsection{The Lie Algebra $\mathfrak{g}$ of a Lie Group $\mathcal{G}$}

The Lie algebra $\mathfrak{g}$ of a Lie group $\mathcal{G}$ is defined as the space of left invariant vector fields on $\mathcal{G}$, which is isomorphic to $T_{e} \mathcal{G}$ the tangent space at the identity $e$. Let $\mathfrak{X}(\mathcal{G})$ denote the space of all vector fields on $\mathcal{G}$, which is an infinite dimensional Lie algebra with Lie bracket given by the commutator bracket $[X, Y]=X Y-$ $Y X$ for $X, Y \in \mathfrak{X}(\mathcal{G})$. A vector field $X \in \mathfrak{X}(\mathcal{G})$ is called left invariant iff $L_{g^{*}} X=X$. If $X, Y$ are left invariant, then $L_{g^{*}}[X, Y]=\left[L_{g^{*}} X, L_{g^{*}} Y\right]=[X, Y]$, i.e. $[X, Y]$ is left invariant. Hence the space of left invariant vector fields $\mathfrak{X}_{L}(\mathcal{G})$ is a Lie subalgebra of $\mathfrak{X}(\mathcal{G})$. 
The Lie algebra $\mathfrak{g}$ of $\mathcal{G}$ is defined as $\mathfrak{g}=\mathfrak{X}_{L}(\mathcal{G})$. Let $\xi \in T_{e} \mathcal{G}$, then $X_{\xi}(g):=$ $T_{e} L_{g}(\xi) \in T_{g} \mathcal{G}$ defines a left invariant vector field and $X_{\xi}(e)=\xi$. This defines a isomorphism between $T_{e} \mathcal{G}$ and $\mathfrak{X}_{L}(\mathcal{G})$. We define the Lie bracket for any $\xi, \eta \in$ $T_{e} \mathcal{G}$ by

$$
[\xi, \eta]:=\left[X_{\xi}, X_{\eta}\right](e)
$$

This bracket satisfies the conditions for a Lie algebra, i.e.[, ] is

- bilinear: $\left[t \xi_{1}+s \xi_{2}, \eta\right]=t\left[\xi_{1}, \eta\right]+s\left[\xi_{2}, \eta\right] \quad t, s \in \mathbb{R}$

- skew symmetric: $[\xi, \eta]=-[\eta, \xi]$

- Jacobi identity $[[\xi, \eta], \zeta]+[[\eta, \zeta], \xi]+[[\zeta, \xi], \eta]=0$ is satisfied.

With this we can identify $\mathfrak{g} \simeq T_{e} \mathcal{G}$ as Lie algebras.

\subsubsection{The Exponential Map}

The exponential map $\exp : T_{e} \mathcal{G} \simeq \mathfrak{g} \rightarrow \mathcal{G}$ is defined as follows: For $\xi \in T_{e} \mathcal{G}$ let $X_{\xi}$ be the associated left invariant vector field. Let $\varphi_{\xi}(t)$ be the flow of $X_{\xi}$ through $e \in \mathcal{G}$ i.e. $\varphi_{\xi}(0)=e, \dot{\varphi}_{\xi}(t)=X_{\xi}(\varphi(t))$. Then we define

$$
\exp (\xi):=\varphi_{\xi}(1)
$$

If $\mathcal{G}$ is finite dimensional then exp defines a local diffeomorphism from a neighborhood of $0 \in \mathfrak{g}$ onto a neighborhood of $e \in \mathcal{G}$. So in finite dimensions, the exponential map defines a local chart about $e \in \mathcal{G}$ (called canonical chart), hence the differential structure of $\mathcal{G}$. In infinite dimensions this is not the case in general.

\subsection{Classical Lie Groups}

We examine these structures and constructions on some examples.

\subsubsection{Vector Groups}

Let $\mathcal{G}=V$ be a Banach space with $\mu(x, y)=x+y, \nu(x)=-x, e=0$, which makes $\mathcal{G}$ into an abelian Lie group, i.e. $\mu(x, y)=\mu(y, x)$. For the Lie algebra we have $\mathfrak{g} \simeq T_{e} V \simeq V$. For $u \in T_{e} V$ the corresponding left invariant vector field $X_{u}$ is given by $X_{u}(v)=u, \forall v \in V$, i.e. $X_{u}=$ const. Hence the Lie algebra $\mathfrak{g}=V$ with the trivial Lie bracket $[u, v]=0$ is abelian. For the exponential map we get $\exp : V \rightarrow V, \exp =\operatorname{id}_{V}$. 


\subsubsection{Matrix Groups}

A) The general linear group $G L(n, \mathbb{R})$ is defined by

$$
\mathcal{G}=G L(n, \mathbb{R}):=\left\{A \in L\left(\mathbb{R}^{n}, \mathbb{R}^{n}\right) ; \operatorname{det} A \neq 0\right\}
$$

which is just the group of invertible $n \times n$ matrices. The determinant map det : $L\left(\mathbb{R}^{n}, \mathbb{R}^{n}\right) \rightarrow \mathbb{R}$ is continuous, hence $G L(n, \mathbb{R})=\operatorname{det}^{-1}(\mathbb{R} \backslash\{0\}) \subset L\left(\mathbb{R}^{n}, \mathbb{R}^{n}\right)$ is an open submanifold. It is disconnected and $\operatorname{dim} G L(n, \mathbb{R})=n^{2}$. The group multiplication $\mu(A, B)=A B$ (matrix multiplication) is $C^{\infty}$ as the restriction of the continuous bilinear map $(A, B) \in L\left(\mathbb{R}^{n}, \mathbb{R}^{n}\right) \times L\left(\mathbb{R}^{n}, \mathbb{R}^{n}\right) \rightarrow A B \in$ $L\left(\mathbb{R}^{n}, \mathbb{R}^{n}\right)$. The group inversion $\nu(A)=A^{-1}$ (matrix inversion) is $C^{\infty}$ by the implicit function theorem, indeed $\mu(A, \nu(A))=e=I$ the identity.

The Lie algebra: Since $G L(n, \mathbb{R}) \subset L\left(\mathbb{R}^{n}, \mathbb{R}^{n}\right)$ is open $T_{I} G L(n, \mathbb{R}) \simeq$ $L\left(\mathbb{R}^{n}, \mathbb{R}^{n}\right)$ with the Lie bracket $[A, B]=A B-B A$. For $A \in L\left(\mathbb{R}^{n}, \mathbb{R}^{n}\right)$ the corresponding vector field $X_{A}$ on on $G L(n, \mathbb{R})$ given by $X_{A}: G L(n, \mathbb{R}) \rightarrow$ $L\left(\mathbb{R}^{n}, \mathbb{R}^{n}\right), X_{A}(Y)=Y A$ is left invariant (linear), indeed $X_{A}\left(L_{Z} Y\right)=X_{A}(Z Y)$ $=Z Y A=\left(T_{Y} L_{Z}\right) X_{A}(Y)$. Hence the Lie bracket on $T_{I} G L(n, \mathbb{R})$ defined by $[A, B]=\left[X_{A}, X_{B}\right](I)=D X_{B}(I) X_{A}(I)-D X_{A}(I) X_{B}(I)$. Since $X_{B}(Z)$ is linear we have $D X_{B}(I) Z=Z B$ and $D X_{B}(I) X_{A}(I)=A B$. So $[A, B]=$ $A B-B A$ is the the usual commutator bracket.

The exponential map: For $A \in L\left(\mathbb{R}^{n}, \mathbb{R}^{n}\right)$ the curve $\gamma_{A}: \mathbb{R} \rightarrow G L(n, \mathbb{R})$ : $\gamma_{A}(t)=\sum_{i=0}^{\infty} \frac{t^{i}}{i !} A^{i}$ is a one-parameter subgroup with $\gamma_{A}(0)=I$ and $\dot{\gamma}_{A}(t)=$ $\sum_{i=1}^{\infty} \frac{t^{i-1}}{(i-1) !} A^{i}=\gamma_{A}(t) A$. Hence $\gamma_{A}$ is the (unique) integral curve of $X_{A}$ and the exponential map $\exp : L\left(\mathbb{R}^{n}, \mathbb{R}^{n}\right) \rightarrow G L(n, \mathbb{R}): \exp (A)=\gamma_{A}(1)=$ $\sum_{i=0}^{\infty} \frac{1}{i !} A^{i}$, becomes $\exp (A)=e^{A}$.

\subsubsection{Lie Subgroups of $G L(n, \mathbb{R})$}

A) The special linear group $S L(n, \mathbb{R})$ is defined as

$$
S L(n, \mathbb{R}):=\{A \in G L(n, \mathbb{R}) ; \operatorname{det} A=1\}=\operatorname{det}^{-1}\{1\} .
$$

$S L(n, \mathbb{R})$ is a closed Lie subgroup of $G L(n, \mathbb{R})$. It is non-compact, connected and $\operatorname{dim} S L(n, \mathbb{R})=n^{2}-1$.

The Lie algebra: $\mathfrak{s l}(n, \mathbb{R})=\left\{A \in L\left(\mathbb{R}^{n}, \mathbb{R}^{n}\right) ;\right.$ trace $\left.A=0\right\}$ with the commutator bracket $[A, B]=A B-B A$. 
B) The orthogonal group $O(n)$ is defined as

$$
O(n):=\left\{A \in L\left(\mathbb{R}^{n}, \mathbb{R}^{n}\right) ;<A x, A y>=<x, y>\Leftrightarrow A A^{T}=I\right\} .
$$

$A \in O(n) \Rightarrow \operatorname{det} A= \pm 1 . O(n)$ is compact, disconnected (2 components) and $\operatorname{dim} O(n)=n(n-1) / 2$.

The Lie algebra: $\mathfrak{o}(n, \mathbb{R})=\left\{A \in L\left(\mathbb{R}^{n}, \mathbb{R}^{n}\right) ; A\right.$ skew symmetric $\}$.

C) The special orthogonal group $S O(n)$ is defined as

$$
\begin{aligned}
S O(n) & =\left\{A \in L\left(\mathbb{R}^{n}, \mathbb{R}^{n}\right) ; A A^{T}=I \& \operatorname{det} A=+1\right\} \\
& =O(n) \cap S L(n, \mathbb{R}) \equiv \text { Identity component of } O(n) .
\end{aligned}
$$

$S O(n)$ is compact, connected and $\operatorname{dim} S O(n)=\frac{1}{2} n(n-1)$.

The Lie algebra: $\mathfrak{s o}(n, \mathbb{R})=\left\{A \in L\left(\mathbb{R}^{n}, \mathbb{R}^{n}\right) ; A\right.$ skew symmetric $\}=\mathfrak{o}(n, \mathbb{R})$.

D) The symplectic group $S p(2 n)$ is defined as

$$
S p(2 n, \mathbb{R})=\left\{A \in L\left(\mathbb{R}^{2 n}, \mathbb{R}^{2 n}\right) ; A^{T} J A=J\right\}
$$

where $J=\left(\begin{array}{cc}O & I \\ -I & 0\end{array}\right) \cdot S p(2 n, \mathbb{R})$ is noncompact and $\operatorname{dim} S p(2 n)=2 n^{2}+n$.

The Lie algebra: $\mathfrak{s p}(2 n, \mathbb{R})=\left\{A \in L\left(\mathbb{R}^{n}, \mathbb{R}^{n}\right) ; A^{T} J+J A=0\right\}$. Similar constructions and results hold for the complex matrix groups $G L(n, \mathbb{C})$ and their corresponding subgroups.

\subsection{Classical Results in Finite Dimensions which are NOT True in Infinite Dimensions:}

1) There is NO Implicit Function Theorem or Inverse Function Theorem in infinite dimensions (except Nash-Moser type theorems!).

2) The exponential map $\exp : \mathfrak{g} \rightarrow G$ defined by $\exp (\xi)=\varphi_{\xi}(1)$, where $\varphi_{\xi}(t)$ is the flow of the left invariant vector field $X_{\xi}$ for $\xi \in \mathfrak{g}$, is a local diffeomorphism in finite dimensions $\Rightarrow$ canonical coordinates. This is not true in infinite dimensions. 3) If $G, H$ are finite dimensional Lie groups and $f_{1}, f_{2}: G \rightarrow H$ are smooth Lie group homomorphisms $\left(G\right.$ connected) i.e. $f_{i}(g h)=f_{i}(g) \cdot f_{i}(h), i=1,2$, with $T_{e} f_{1}=T_{e} f_{2}$, then locally $f_{1}=f_{2}$.

4) If $f: G \rightarrow H$ is a continuous group homomorphism between finite dimensional Lie groups then $f$ is smooth.

5) If $G$ is a finite dimensional Lie group and $H \subset G$ a closed subgroup then $H$ is 
a Lie subgroup (Lie group and submanifold).

6) If $G$ is a finite dimensional Lie group with Lie algebra $\mathfrak{g}$ and $\mathfrak{h} \subset \mathfrak{g}$ is a subalgebra, then there exists a unique connected Lie subgroup $H \subset G$ with $\mathfrak{h}$ as its Lie algebra, i.e $\mathfrak{h} \simeq T_{e} H$.

7) If $\mathfrak{g}$ is any finite dimensional Lie algebra the there exists a connected finite dimensional Lie group $G$ with $\mathfrak{g}$ as its Lie algebra i.e. $\mathfrak{g} \simeq T_{e} G$.

\subsection{Infinite Dimensional Examples}

\subsubsection{The General Linear Group}

Let $V$ be a Banach space and $L(V, V)$ the space of bounded linear operators $A: V \rightarrow V$. Then $L(V, V)$ is a Banach space with the operator norm $\|A\|=$ $\sup _{\|x\|<1}\|A(x)\|$ and the group $\mathcal{G}=G L(V, V)$ of invertible elements is open in $L(V, V)$. So $G L(V, V)$ is a smooth Lie group with $\mu(f, g)=f \circ g, \nu(f)=$ $f^{-1}, e=\mathrm{id}_{V}$. Its Lie algebra is $\mathfrak{g}=L(V, V)$ with the commutator bracket $[A, B]=A B-B A$ and exponential map $\exp A=e^{A}$.

\subsubsection{Abelian Gauge Groups with Application}

Let $M$ be finite dimensional manifold and $\mathcal{G}=C^{\infty}(M)$ with $\mu(f, g)=f+g$ and $\nu(f)=-f, e=0$. This is an infinite dimensional (abelian) vector group. The Lie algebra is $\mathfrak{g}=T_{e} C^{\infty}(M) \simeq C^{\infty}(M)$, with trivial bracket $[\xi, \eta]=0$ (abelian) and exponential map exp $=$ id $: C^{\infty}(M) \rightarrow C^{\infty}(M)$. This is a $C^{\infty}$. Frechet Lie group (vector group). We can norm-complete $C^{\infty}(M)$ with respect to the $C^{k}$-norm, $k<\infty$, and obtain the Banach Lie group $C^{k}(M)$, or with respect to the $H^{s}$-Sobolev norm, $s>\frac{1}{2} \operatorname{dim} M$, and obtain the Hilbert Lie group $H^{s}(M)$. For example, if $(M, g)$ is a Riemannian manifold with Levi-Civita connection $\nabla$ the $H^{s}(M)$ is the completion of $C^{\infty}(M)$ with respect to the norm $\|f\|_{s}=$ $\left(\int_{M} \sum_{i=1}^{s}\left|\nabla^{i} f\right|_{x}^{2} \mathrm{~d} v o l_{x}(g)\right)^{1 / 2}<\infty$.

\section{Application: Maxwell's Equations}

Let $E, B$ be the electric and magnetic fields respectively. Then the vacuum Maxwell equations are

$$
\dot{E}=\operatorname{curl} B, \quad \dot{B}=-\operatorname{curl} E, \quad \operatorname{div} B=0, \operatorname{div} E=\rho .
$$

Let $V$ be the space of vector fields (potentials) on $\mathbb{R}^{3}$ and the phase space $P=$ $T^{*} V=V \times V^{*} \ni(A, E)$, with the $L^{2}$ pairing $\langle A, E\rangle=\int A(x) E(x) \mathrm{d} x$. The 
canonical Poisson bracket for $F, H: P \rightarrow \mathbb{R}$ is given by

$$
\{F, H\}(A, E)=\int\left(\frac{\delta F}{\delta A} \frac{\delta H}{\delta E}-\frac{\delta H}{\delta A} \frac{\delta F}{\delta E}\right) \mathrm{d} x .
$$

The canonical Hamilton equations on $P=T^{*} V$ are

$$
\dot{A}=\frac{\delta H}{\delta E}, \quad \dot{E}=-\frac{\delta H}{\delta A} \text {. }
$$

With the Hamiltonian (energy) $H(A, E)=\frac{1}{2} \int\left(|\operatorname{curl} A|^{2}+|E|^{2}\right) \mathrm{d} x$ and $B:=$ $-\operatorname{curl} A$ we get $\operatorname{div} B=-\operatorname{div} \operatorname{curl} A=0$ and Hamiltons equations $\dot{A}=\frac{\delta H}{\delta E}=E \Rightarrow$ $\dot{B}=-\operatorname{curl} E$ and $\dot{E}=-\frac{\delta H}{\delta A}=-\operatorname{curl} \operatorname{curl} A=\operatorname{curl} B$. These are the first three of Maxwells' equations, the fourth equation $\operatorname{div} E=\rho$ is obtained from gauge symmetry and reduction.

Gauge invariance: The Lie group $\mathcal{G}=C^{\infty}\left(\mathbb{R}^{3}\right)$ acts on $V$ by $\varphi \cdot A=A+\nabla \varphi$ $(\varphi \in \mathcal{G}, A \in V)$. We lift this action to $P=V \times V^{*}$ as follows $\varphi \cdot(A, E)=$ $(A+\nabla \varphi, E)$. The Hamiltonian $H$ is $\mathcal{G}$ invariant and has a momentum map $J: V \times V^{*} \rightarrow \mathfrak{g}^{*}$ given by $J(A, E)=\operatorname{div} E$. We identify the dual of the Lie algebra $\mathfrak{g}^{*}$ with charge densities on $\mathbb{R}^{3}$. The reduced phase space for $\rho \in \mathfrak{g}^{*}$ becomes $P_{\rho}=J^{-1}(\rho) / \mathcal{G}=\{(E, B) ; \operatorname{div} E=\rho, \operatorname{div} B=0\}$ and the reduced Hamiltonian becomes $H_{\rho}(E, B)=\frac{1}{2} \int\left(|E|^{2}+|B|^{2}\right) \mathrm{d} x$. Computing the reduced Poisson bracket on $P_{\rho}$ we get

$$
\{F, H\}_{\rho}(E, B)=\int\left(\frac{\delta F}{\delta E} \cdot \operatorname{curl} \frac{\delta H}{\delta B}-\frac{\delta H}{\delta E} \cdot \operatorname{curl} \frac{\delta F}{\delta B}\right) \mathrm{d} x .
$$

Now Hamilton's equations on the reduced phase $P_{\rho}$ space become Maxwell's equations

$$
\dot{F}=\left\{F, H_{\rho}\right\}_{\rho} \Leftrightarrow \begin{cases}\dot{E}=\operatorname{curl} B, & \dot{B}=-\operatorname{curl} E \\ \operatorname{div} B=0, & \operatorname{div} E=\rho .\end{cases}
$$

\subsubsection{Loop Groups}

Let $M$ be a finite dimensional manifold and $\mathcal{G}=C^{\infty}(M, \mathbb{R} \backslash\{0\})$ with $\mu(f, g)=$ $f \cdot g$ and $\nu(f)=f^{-1}, e=1 . C^{k}(M, \mathbb{R} \backslash\{0\})$ is open in $C^{\infty}(M, \mathbb{R})$. If $M$ is compact then $C^{k}(M, \mathbb{R} \backslash\{0\})$ is a Banach Lie group for $k<\infty$ and $H^{s}(M, \mathbb{R} \backslash\{0\})$ 
(diffeomorphisms of $H^{s}$ Sobolev class) is closed under multiplication if $s>$ $\frac{1}{2} \operatorname{dim} M$. Then $H^{s}(M, \mathbb{R} \backslash\{0\})$ is a Hilbert Lie group.

As a generalization we replace $\mathbb{R}-\{0\}$ by any finite dimensional Lie group $G$ with Lie algebra $\mathbf{g}$. Then $\mathcal{G}=C^{k}(M, G)$ with pointwise defined multiplication and inversion, (i.e. for $f, g \in \mathcal{G}, x \in M, \mu(f, g)(x)=f(x) \cdot g(x)$ and $\nu(f)(x)=$ $\left.f^{-1}(x)\right)$ is a Banach Lie group with Lie algebra $\mathfrak{g}=C^{k}(M, \mathbf{g})$ where the bracket is again defined pointwise $[\xi, \eta]_{\mathfrak{g}}(x)=[\xi(x), \eta(x)]_{\mathbf{g}}$ for $\xi, \eta \in \mathfrak{g}, x \in M$.

The exponential map $\operatorname{Exp}: \mathfrak{g}=C^{k}(M, \mathbf{g}) \rightarrow \mathcal{G}=C^{k}(M, G)$ is given by $\operatorname{Exp}(\xi)=\exp \circ \xi$, where $\exp : \boldsymbol{g} \rightarrow G$ is the finite dimensional exponential map. In this case Exp is a local diffeomorphism.

Special case: If $M=S^{1}$ the circle then $\mathcal{G}=C^{k}\left(S^{1}, G\right)=L^{k}(G)$ is known as the loop group and $\mathfrak{g}=C^{k}\left(S^{1}, \mathbf{g}\right)=\mathfrak{l}^{k}(\mathbf{g})$ its loop algebra, see [31] for details.

Applications: These infinite dimensional Lie groups have wide applications in gauge theories and quantum field theory. Their Lie algebras and their representation theory play an important role to affine Lie algebras, Kac-Moody Lie algebras (central extensions), vertex algebras, completely integrable systems and soliton equations (Toda, KdV, KP equations).

\section{Diffeomorphism groups}

\subsection{Overview of $\operatorname{Diff}(M)$}

Diffeomorphism groups and their subgroups provide an important and wide range of examples of infinite dimensional Lie groups with very interesting applications to mathematical physics. Let $M$ be a smooth manifold, and consider

$$
\operatorname{Diff}(M)=\{f: M \rightarrow M ; f \text { diffeomorphism }\} .
$$

\subsubsection{The Algebraic Structure of $\operatorname{Diff}(M)$}

$\operatorname{Diff}(M)$ is a group with composition as group operation

$$
\mu: \operatorname{Diff}(M) \times \operatorname{Diff}(M) \rightarrow \operatorname{Diff}(M), \mu(f, g)=f \circ g
$$

and inversion

$$
\nu: \operatorname{Diff}(M) \rightarrow \operatorname{Diff}(M), \nu(f)=f^{-1} .
$$

The unit element $e$ is the identity map $e=\operatorname{id}_{M}: M \rightarrow M$. We call $\operatorname{Diff}(M)$ the diffeomorphism group of $M$. There are very interesting algebraic properties like 
cohomologies, isomorphy types, simplicity ect. studied mainly by A. Banyaga in the 1970's, [5]. We concentrate here on the geometric structures and its applications.

\subsubsection{Geometric Structure of $\operatorname{Diff}(M)$}

We investigate the topology, the manifold structure and global analysis on $\operatorname{Diff}(M)$. One of the main question is, in what sense is $\operatorname{Diff}(M)$ a Lie group?

For different types of diffeomorphisms we will obtain different structures:

- $C^{k}, k<\infty$ diffeomorphisms $\Rightarrow \operatorname{Diff}^{k}(M)$ is a Banach manifold (Palais [30], Omori [28]).

- $C^{\infty}$ diffeomorphisms $\Rightarrow \operatorname{Diff}^{\infty}(M)$ is a Frechet manifold (Omori [28], Schmid [35]).

- $H^{s}$ Sobolev $H^{s}$ diffeomorphims, $f$ is $H^{s}$ iff in any local chart all derivatives of $f$ up to order $s$ are square integrable, $s>\frac{1}{2} \operatorname{dim} M \Rightarrow \operatorname{Diff}^{s}(M)$ is a Hilbert manifold (Ebin-Marsden[13], Ratiu-Schmid [32] and EichhornSchmid [15]).

In a nut shell we will prove the following result:

Theorem 1. Diff ${ }^{s}(M)$ is a smooth infinite dimensional manifold and a Lie group in the following sense: The group multiplication $\mu$ is $C^{k}$ as a map

$$
\mu: \operatorname{Diff}^{s+k}(M) \times \operatorname{Diff}^{s}(M) \rightarrow \operatorname{Diff}^{s}(M), \mu(f, g)=f \circ g .
$$

The inversion $\nu$ is $C^{k}$ as a map

$$
\nu: \operatorname{Diff}^{s+k}(M) \rightarrow \operatorname{Diff}^{s}(M), \nu(f)=f^{-1} .
$$

Note that the group operations are not smooth between the same spaces, i.e. if $k=0$ they are only continuous. In order to obtain smoothness to some degree one must change the space to that degree. As $s \rightarrow \infty$ we obtain Diff ${ }^{\infty}(M)=$ $\underset{\leftarrow}{\lim } \operatorname{Diff}^{s}(M)$ as inverse limit a "Frechet Lie group" called ILH Lie group (Inverse Limit of Hilbert Lie groups). We give the exact definition and properties below.

\subsection{The Manifold Structure on $C(M, M)$}

We'll see that the diffeomorphism group $\operatorname{Diff}(M)$ is an open set of the manifold of all maps $C(M, M)$. So we first need to construct a manifold structure on the spaces of maps $C^{\infty}(M, M), C^{k}(M, M)$ and $C^{s}(M, M)$. 
Let $M$ be a compact and $N$ any finite dimensional manifold and consider

$$
C(M, N)=\{f: M \rightarrow N ; f \text { differentiable }\} .
$$

We first consider two different manifolds $M$ and $N$ to make the construction clearer, later we'll set $N=M$. Also we do not specify at the moment the differentiability class $C^{\infty}, C^{k}$ or $H^{s}$.

\subsubsection{The Parameter Spaces of $C(M, N)$}

In order to construct an infinite dimensional manifold structure on a set like $C(M, N)$ one first has to find a candidate for the parameter spaces. As for finite dimensional manifolds, these parameter spaces should be isomorphic to the tangent spaces at the corresponding points. Heuristically one determines the tangent space as follows:

Let $f \in C(M, N)$ and $\gamma: I \subset \mathbb{R} \rightarrow C(M, N)$ be a curve with $\gamma(0)=f$. Then $\dot{\gamma}(0)$ is a tangent vector to $C(M, N)$ at the point $f$, i.e.

$$
\dot{\gamma}(0)=\left.\frac{\mathrm{d} \gamma(t)}{\mathrm{d} t}\right|_{t=0} \in T_{f} C(M, N) .
$$

We make the following identification to interpret $\dot{\gamma}(0)$ : For each $x \in M$ let $\gamma_{x}$ : $I \subset \mathbb{R} \rightarrow N$ be the curve in $N$ defined by $\gamma_{x}(t)=\gamma(t)(x)$. Then $\gamma_{x}(0)=f(x)$ and $\dot{\gamma}_{x}(0) \in T_{f(x)} N$, i.e. $\dot{\gamma}_{x}(0)$ is a tangent vector to $N$ at the point $f(x)$. We identify $\dot{\gamma}_{x}(0) \equiv \dot{\gamma}(0)(x)$; hence we can regard $\dot{\gamma}(0)$ as a map $\dot{\gamma}(0): M \rightarrow T N$ such that $\dot{\gamma}(0)(x) \in T_{f(x)} N$. That means $\dot{\gamma}(0)$ is a vector field along $f$. With this motivation we define the tangent space of $C(M, N)$ at the point $f \in C(M, N)$ by

$$
T_{f} C(M, N)=\left\{\xi_{f} \in C(M, T N) ; \tau_{N} \circ \xi_{f}=f\right\}
$$

where $\tau_{n}: T N \rightarrow N$ is the canonical projection. We can identify $T_{f} C(M, N)$ with the space $\Gamma\left(f^{*} \tau_{N}\right)$ of sections of the pull back bundle $f^{*} \tau_{N}$. This is the parameter space at the point $f \in C(M, N)$, i.e. $T_{f} C(M, N) \cong \Gamma\left(f^{*} \tau_{N}\right)$ which is an infinite dimensional vector space.

Note, for $N=M$ and $f=$ id $: M \rightarrow M$ we have $T_{\text {id }} C(M, M)=\Gamma\left(\tau_{M}\right)=$ $\mathfrak{X}(M)$ the space of vector fields on $M$.

For different differentiability types of $f: M \rightarrow N$ we choose the corresponding topologies on the vector spaces $\Gamma\left(f^{*} \tau_{M}\right)$ as follows:

a) $C^{\infty}(M, N)$ : The space $\Gamma^{\infty}\left(f^{*} \tau_{M}\right)$ of $C^{\infty}$ sections with the uniform $C^{\infty}$ topology is a Frechet space; i.e. a metrizable topological vector space. This 
topology is defined by the sequence of seminorms $\left(\mid b_{p}\right)_{p \in \mathbb{N}}$

$$
|\xi|_{p}=\max _{0 \leq i \leq p, 0 \leq j \leq p} \sup _{x \in U_{i}}\left\|D^{j} \xi_{i}(x)\right\|<\infty
$$

where $\xi_{i}$ is the local representative of $\xi \in \Gamma^{\infty}\left(f^{*} \tau_{M}\right)$ in a chart $U_{i}$ of $M$. Note there is no single norm defining this topology, but it is metrizable and complete. b) $C^{k}(M, N), 0 \leq k<\infty$ : The space $\Gamma^{k}\left(f^{*} \tau_{M}\right)$ of $C^{k}$-differentiable sections with the uniform $C^{k}$-topology is a Banach space with norm

$$
\|\xi\|=\max _{0 \leq p \leq k}|\xi|_{p}, \text { with }|\xi|_{p} \text { as above. }
$$

c) $C^{s}(M, N), s>\frac{1}{2} \operatorname{dim} M:$ The space $\Gamma^{s}\left(f^{*} \tau_{M}\right)$ of $H^{s}$-Sobolev differentiable sections is a Hilbert space with inner product

$$
<\xi, \eta>=\max _{i} \int_{U_{i}}\left(\sum_{0 \leq|k| \leq s} D^{k} \xi_{i}(x) D^{k} \eta_{i}(x)\right) \mathrm{d} x .
$$

\subsubsection{Local Charts (coordinates) of $C(M, M)$}

Now let $N=M$ in the above construction. We choose a Riemannian metric on $M$, which allows us to define a (Riemannian) exponential map exp :TM $\rightarrow M$ as follows. For each $v_{x} \in T_{x} M$ there is a unique geodesic $\alpha_{x}$ through $x$ whose tangent vector at $x$ is $v_{x}$, i.e $\alpha_{x}(0)=x$ and $\dot{\alpha}_{x}(0)=v_{x}$. Then define

$$
\exp _{x}\left(v_{x}\right):=\alpha_{x}(1), v_{x} \in T_{x} M .
$$

In general $\exp _{x}$ is a local diffeomorphism from a neighborhood of $0 \in T_{x} M$ onto a neighborhood of $x \in M$. However, since $M$ is compact, $\exp _{x}$ is defined on all of $T_{x} M$ and can be extended to a map exp :TM $\rightarrow M$ such that the map

$$
\operatorname{Exp}:=\left(\tau_{M}, \exp \right): T M \rightarrow M \times M, \operatorname{Exp}\left(v_{x}\right)=\left(x, \exp _{x}\left(v_{x}\right)\right)
$$

is a diffeomorphism from a neighborhood $\mathcal{O}(0)$ of the zero section in $T M$ onto neighborhood $\mathcal{U}(\Delta)$ of diagonal $\Delta \subset M \times M$,

$$
\operatorname{Exp}: T M \supset \mathcal{O}(0) \simeq \mathcal{U}(\Delta) \subset M \times M .
$$

We define $f: M \rightarrow M$ to be close to the identity id $: M \rightarrow M$ iff $\operatorname{graph}(f) \subset$ $\mathcal{U}(\Delta)$. This defines a neighborhood $\mathcal{V}\left(\operatorname{id}_{M}\right)$ in $C(M, M)$ by

$$
\mathcal{V}\left(\operatorname{id}_{M}\right)=\{f \in C(M, M) ; \operatorname{graph}(f) \subset \mathcal{U}(\Delta)\} .
$$


We define $\xi: M \rightarrow T M$ to be close to the zero section $0: M \rightarrow T M$ iff $\xi(M) \subset \mathcal{O}(0)$. This defines a neighborhood $\mathcal{W}(0)$ in $\mathfrak{X}(M)=T_{\mathrm{id}} C(M, M)$ by

$$
\mathcal{W}(0)=\{\xi \in C(M, T M) ; \xi(M) \subset \mathcal{O}(0)\} .
$$

We want to point out that compactness of $M$ is important. We have the following lemma:

Lemma 2. If $\tau: E \rightarrow M$ is any vector bundle over $M$, denote by $\Gamma(\tau)=\{\xi:$ $M \rightarrow E ; \tau \circ \xi=\mathrm{id}\}$ the space of sections. Let $\mathcal{O} \subset E$ be an open set of $E$. Then the set $\Gamma(\mathcal{O}):=\{\xi \in \Gamma(\tau) ; \xi(M) \subset \mathcal{O}\}$ is open in $\Gamma(\tau)$ (open in any of the topologies $\left.\Gamma^{\infty}, \Gamma^{k}, \Gamma^{s}\right)$ if and only if $M$ is compact.

From this lemma follows that the neighborhood $\mathcal{W}(0)$ defined above is open in $\Gamma\left(\tau_{M}\right)=\mathfrak{X}(M)=T_{\mathrm{id}} C(M, M)$.

Define $\Phi: \mathcal{V}\left(\operatorname{id}_{M}\right) \subset C(M, M) \rightarrow \mathcal{W}(0) \subset \mathfrak{X}(M)$ by

$$
\Phi(f)=\operatorname{Exp}^{-1} \circ\left(\mathrm{id}_{M}, f\right), f \in \mathcal{V}\left(\mathrm{id}_{M}\right) .
$$

$\Phi$ is a bijection from $\mathcal{V}\left(\operatorname{id}_{M}\right)$ onto $\mathcal{W}(0)$ with inverse

$$
\Phi^{-1}(\xi)=p r_{2} \circ \operatorname{Exp} \circ \xi, \xi \in \mathcal{W}(0)
$$

where $p r_{2}: M \times M \rightarrow M$ is the projection onto the second factor.

Therefore we have a chart about the identity $\operatorname{id}_{M}$ on $C(M, M)$

$$
\Phi: \mathcal{V}\left(\operatorname{id}_{M}\right) \subset C(M, M) \rightarrow \mathcal{W}(0) \subset \Gamma\left(\tau_{M}\right)
$$

A similar construction yields charts about any $f \in C(M, M)$, i.e. there exists open neighborhoods $\mathcal{W}_{f}$ in $\Gamma\left(f^{*} \tau_{M}\right)$ and $\mathcal{V}_{f}$ in $C(M, M)$ and bijections $\Phi_{f}$ : $\mathcal{V}_{f} \rightarrow \mathcal{W}_{f}$ where $\Phi_{f}(g)=\operatorname{Exp}_{f}^{-1} \circ($ id $M, g)$ with $\operatorname{Exp}_{f}=\left(\tau_{f^{*} M}, \exp \circ f\right)$.

\subsubsection{Change of Coordinates:}

The change of charts (change of coordinates) for any $f, g \in C(M, N)$ is given by the map

$$
\Phi_{f, g}=\Phi_{g} \circ \Phi_{f}^{-1}: \Phi_{f}\left(\mathcal{V}_{f} \cap \mathcal{V}_{g}\right) \subset \Gamma\left(f^{*} \tau_{M}\right) \rightarrow \Gamma\left(g^{*} \tau_{M}\right)
$$

which turns out to be

$$
\Phi_{f, g}\left(\xi_{f}\right)=\operatorname{Exp}_{g}^{-1} \circ \operatorname{Exp}_{f} \circ \xi_{f}
$$


where $\operatorname{Exp}_{f}=\left(\tau_{f^{*} M}\right.$, $\left.\exp \circ f\right)$ and $\operatorname{Exp}_{g}=\left(\tau_{g^{*} M}, \exp \circ g\right)$ are the from Exp induced local diffeomorphisms in the bundles $\Gamma\left(f^{*} \tau_{M}\right)$ and $\Gamma\left(g^{*} \tau_{M}\right)$ respectively. To show that these transition maps $\Phi_{f, g}$ are smooth one needs the $\Omega$-Lemma (Palais [30]).

Lemma 3. ( $\Omega$-Lemma) Let $\tau: E \rightarrow M$ and $\tau^{\prime}: E^{\prime} \rightarrow M$ be vector bundles over $M$ (compact!) and let $\mathcal{O} \subset E$ be open. Let $\omega: \mathcal{O} \subset E \rightarrow E^{\prime}$ be a $C^{\infty}$-vector bundle map. Then the induced map

$$
\Omega_{\omega}: \Gamma(\mathcal{O}) \subset \Gamma(\tau) \rightarrow \Gamma\left(\tau^{\prime}\right), \Omega_{\omega}(\xi):=\omega \circ \xi
$$

is a $C^{\infty}$ map, with derivatives $D^{k} \Omega_{\omega}=\Omega_{\partial^{k} \omega}$, i.e $D^{k} \Omega_{\omega}(\xi)=\partial^{k} \omega \circ \xi$, where $\partial^{k} \omega$ denotes the fiber derivative.

Remarks: For the different topologies $\Gamma^{\infty}, \Gamma^{k}, \Gamma^{s}$ we have:

a) $\Gamma^{\infty}$ : The differentiability of the mapping $\Omega_{\omega}$ between the Frechet spaces $\Gamma^{\infty}(\tau)$ and $\Gamma^{\infty}\left(\tau^{\prime}\right)$ is $C_{\sigma_{0}}^{\infty}$ in the sense of Keller [21].

b) $\Gamma^{k}, 0 \leq k<\infty$ : If $\omega$ is $C^{\infty}$ then $\Omega_{\omega}: \Gamma^{k}(\mathcal{O}) \subset \Gamma^{k}(\tau) \rightarrow \Gamma^{k}\left(\tau^{\prime}\right)$ is $C^{\infty}$. If $\omega$ is $C^{k+p}$, then $\Omega_{\omega}: \Gamma^{k}(\mathcal{O}) \subset \Gamma^{k}(\tau) \rightarrow \Gamma^{k}\left(\tau^{\prime}\right)$ is $C^{p}$.

c) $\Gamma^{s}, s>\frac{1}{2} \operatorname{dim} M:$ If $\omega$ is $C^{\infty}$ then $\Omega_{\omega}: \Gamma^{s}(\mathcal{O}) \subset \Gamma^{s}(\tau) \rightarrow \Gamma^{s}\left(\tau^{\prime}\right)$ is $C^{\infty}$. If $\omega$ is Sobolev $H^{s+t}$, then $\Omega_{\omega}: \Gamma^{s}(\mathcal{O}) \subset \Gamma^{s}(\tau) \rightarrow \Gamma^{s}\left(\tau^{\prime}\right)$ is $H^{t}$.

It follows from the $\Omega$-Lemma (3) that the change of charts $\Phi_{f, g}$ is $C^{\infty}$, because $\Phi_{f, g}=\Omega_{\omega}$ for $\omega=\operatorname{Exp}_{g}^{-1} \circ \operatorname{Exp}_{f}: \mathcal{O}_{f} \subset f^{*} T M \rightarrow g^{*} T M$ and $\omega$ is $C^{\infty}$. So we have the following

Theorem 4. Under the above assumptions $C^{\infty}(M, M), C^{k}(M, M), 0 \leq k<\infty$ and $C^{s}(M, M), s>\frac{1}{2} \operatorname{dim} M$ are infinite dimensional $C^{\infty}$ Frechet, Banach and Hilbert manifolds, respectively.

Remark: A similar construction works for the space $C(M, N)$, where $M$ is a compact manifold and $N$ is a Riemannian (not nec. compact) manifold. Again $C^{\infty}(M, N), C^{k}(M, N), 0 \leq k<\infty$ and $C^{s}(M, N), s>\frac{1}{2} \operatorname{dim} M$ are infinite dimensional $C^{\infty}$ Frechet, Banach, Hilbert manifolds.

\subsection{The Diffeomorphism Group Diff $(M)$}

From now on we restrict our attention to the class of Sobolev $H^{s}$ mappings. This is convenient for physical applications because we are working locally in Hilbert 
spaces. Similar results are true for the $C^{k}, 0 \leq k<\infty$, case where locally we have Banach spaces to work with. In these two cases we have the classical inverse mapping theorem and implicit function theorem. In the case of $C^{\infty}$ mappings the situation is quite different. We have to work locally with Frechet spaces and there are no easy generalizations of these theorems available; see Hamilton [20], Nash [27], Moser [26] and Schmid [34].

\subsubsection{The Lie Group Structure of $\operatorname{Diff}^{s}(M)$}

Let $\operatorname{Diff}^{s}(M)$ denote the set of all diffeomorphisms of Sobolev $H^{s}$ class on $M$. This set is well defined if $s>\frac{1}{2} \operatorname{dim} M$ (which we always assume from now on). So $f \in \operatorname{Diff}^{s}(M)$ if and only if $f$ is bijective and $f, f^{-1}: M \rightarrow M$ are of Sobolev class $H^{s}$.

$\operatorname{Diff}^{s}(M)$ is a group with continuous group operations

$$
\begin{aligned}
& \mu: \operatorname{Diff}^{s}(M) \times \operatorname{Diff}^{s}(M) \rightarrow \operatorname{Diff}^{s}(M), \quad \mu(f, g)=f \circ g \\
& \nu: \operatorname{Diff}^{s}(M) \rightarrow \operatorname{Diff}^{s}(M), \nu(f)=f^{-1}
\end{aligned}
$$

the neutral element is $e=\mathrm{id}: M \rightarrow M$. So $\operatorname{Diff}^{s}(M)$ is a topological group.

The question is whether the group operations $\mu$ and $\nu$ are smooth $C^{\infty}$,in order to define a Lie group structure on $\operatorname{Diff}^{s}(M)$. The answer in NO! The situation is more complicated as indicated at the beginning.

Right multiplication: Let $g \in \operatorname{Diff}^{s}(M)$. Right multiplication is the map $R_{g}$ : $\operatorname{Diff}^{s}(M) \rightarrow \operatorname{Diff}^{s}(M)$ defined by $R_{g}(f)=f \circ g$. It's tangent map (derivative) at any $f \in \operatorname{Diff}^{s}(M)$ is the map $T_{f} R_{g}: T_{f} \operatorname{Diff}^{s}(M) \rightarrow T_{f \circ g} \operatorname{Diff}^{s}(M)$ : $T_{f} R_{g}\left(\xi_{f}\right)=\xi_{f} \circ g$. So $T R_{g}=R_{g}$, therefore $R_{g}$ is $C^{\infty}$ for any $g \in \operatorname{Diff}^{s}(M)$.

Left multiplication: Let $g \in \operatorname{Diff}^{s}(M)$. Left multiplication is the map $L_{g}$ : $\operatorname{Diff}^{s}(M) \rightarrow \operatorname{Diff}^{s}(M): L_{g}(f)=g \circ f$. It is tangent map (derivative) at $f \in$ $\operatorname{Diff}^{s}(M)$ is the map $T_{f} L_{g}: T_{f} \operatorname{Diff}^{s}(M) \rightarrow T_{g \circ f} \operatorname{Diff}^{s}(M): T_{f} L_{g}\left(\xi_{f}\right)=T g \circ$ $\xi_{f}$. Therefore $T L_{g}=L_{T g}$. But if $g$ is $H^{s}$ then $T g$ is $H^{s-1}$ therefore $L_{g}$ : $\operatorname{Diff}^{s+k}(M) \rightarrow \operatorname{Diff}^{s}(M)$ is $C^{k}$ for any $g \in \operatorname{Diff}^{s+k}(M)$, ( $(\Omega$-Lemma).

Together we get: The group multiplication $\mu$ is $C^{k}$ as a map

$$
\mu: \operatorname{Diff}^{s+k}(M) \times \operatorname{Diff}^{s}(M) \rightarrow \operatorname{Diff}^{s}(M) .
$$

Note that for $k=0 \Rightarrow$ the multiplication is only continuous between the same spaces $\operatorname{Diff}^{s}(M)$. 
Similarly, the group inversion $\nu$ is $C^{k}$ as a map

$$
\nu: \operatorname{Diff}^{s+k}(M) \rightarrow \operatorname{Diff}^{s}(M)
$$

and $k=0 \Rightarrow$ the inversion is only continuous between the same spaces $\operatorname{Diff}^{f}(M)$.

\subsubsection{The Lie Algebra of $\operatorname{Diff}^{s}(M)$}

Recall that for a finite dimensional Lie group $G$, the Lie algebra structure on $\mathbf{g} \cong$ $T_{e} G$ (e=identity) is defined as follows: for $\xi, \eta \in T_{e} G$ define the corresponding left invariant vector fields $X_{\xi}(g):=T_{e} L_{g}(\xi), X_{\eta}(g):=T_{e} L_{g}(\eta), g \in G$. Then define the Lie bracket on $\mathrm{g}$ by $[\xi, \eta]:=\left[X_{\xi}, X_{\eta}\right](e)$, where $\left[X_{\xi}, X_{\eta}\right]=$ $X_{\xi} X_{\eta}-X_{\eta} X_{\xi}$ is the commutator bracket of $X_{\xi}$ and $X_{\eta}$ which is again a left invariant vector field. Instead of left invariant vector fields one could use right invariant ones and would get anti-isomorphic Lie algebras.

For the diffeomorphism group $\mathcal{G}=\operatorname{Diff}^{s}(M)$ right multiplications is $C^{\infty}$ whereas left multiplication is only continuous, therefore we use right invariant vector fields rather then left invariant ones to define the Lie algebra $\mathfrak{g}$ of $\mathcal{G}=\operatorname{Diff}^{s}(M)$. A right invariant vector field on $\operatorname{Diff}^{s}(M)$ is a map $Y: \operatorname{Diff}^{s}(M) \rightarrow T \operatorname{Diff}^{s}(M)=$ $C^{s}(M, T M)$ such that $Y(f) \in T_{f} \operatorname{Diff}^{s}(M)$, i.e $\tau_{M} \circ X(f)=f$ is a vector field along $f$, and $R_{g}^{*} Y=Y, g \in \operatorname{Diff}^{s}(M)$. Let $\xi \in T_{\text {id }_{M}} \operatorname{Diff}^{s+1}(M)=\mathfrak{X}^{s+1}(M)$ be an $H^{s+1}$-vector fields on $M$, and define the right invariant vector field $Y_{\xi}$ on $\operatorname{Diff}^{s}(M)$ by

$$
Y_{\xi}(f):=T R_{f}(\xi)=\xi \circ f, f \in \operatorname{Diff}^{s}(M) .
$$

$Y_{\xi}$ is a $C^{1}$ vector field on $\operatorname{Diff}^{s}(M)$ if $\xi \in \mathfrak{X}^{s+1}(M)$. The bracket $\left[Y_{\xi}, Y_{\eta}\right]$ of two right invariant vector fields is given by

$$
\left[Y_{\xi}, Y_{\eta}\right]\left(\mathrm{id}_{M}\right)=-[\xi, \eta], \quad \xi, \eta \in \mathfrak{X}^{s+1}(M)
$$

where the bracket $[\xi, \eta]$ is the commutator bracket of the vector fields $\xi$ and $\eta$ on $M$, i.e. $[\xi, \eta]=\xi \eta-\eta \xi$. If $\xi, \eta \in \mathfrak{X}^{s+1}(M)$ the $[\xi, \eta] \in \mathfrak{X}^{s}(M)$, hence $\left[Y_{\xi}, Y_{\eta}\right]\left(\operatorname{id}_{M}\right) \in \mathfrak{X}^{s}(M)$ and $\left[Y_{\xi}, Y_{\eta}\right]$ is a right invariant $C^{0}$ vector field on $\operatorname{Diff}^{s}(M)$. So the ordinary Lie bracket $[\xi, \eta]=\xi \eta-\eta \xi$ of vector fields on $M$ is the bracket on the Lie algebra $\mathfrak{g}=T_{\operatorname{id}_{M}} \operatorname{Diff}^{s+1}(M)$ but $\mathfrak{g}$ is not closed under the bracket as we loose derivatives! This type of Lie algebra structure is called "ILH Lie algebra" which we will define below.

\subsubsection{The Exponential Map exp : $\mathfrak{g} \rightarrow \mathcal{G}$}

Recall for a finite dimensional Lie group $G$ with Lie algebra $\mathrm{g}=T_{e} G$ the exponential map exp : $\mathrm{g} \rightarrow G$ is defined as follows: For $\xi \in \mathrm{g}$ let $X_{\xi}$ be the 
corresponding left invariant vector field and $\varphi_{\xi}(t)$ its integral curve at $e \in G$, i.e. $\varphi_{\xi}: \mathbb{R} \rightarrow G$ smooth, $\varphi_{\xi}(0)=e$ and $\dot{\varphi}_{\xi}(t)=X_{\xi}\left(\varphi_{\xi}(t)\right)$. Now define $\exp (\xi):=\varphi_{\xi}(1)$. Then the map exp is a local diffeomorphism from a neighborhood of zero in $\mathrm{g}$ onto a neighborhood of $e$ in $G$. This is true only in finite dimensions!

For the Lie group $\mathcal{G}=\operatorname{Diff}^{s}(M)$ with Lie algebra $\mathfrak{g}=T_{e} \operatorname{Diff}^{s+1}(M)=\mathfrak{X}^{s+1}(M)$ the exponential map

$$
\exp : T_{e} \operatorname{Diff}^{s}(M)=\mathfrak{X}^{s}(M) \rightarrow \operatorname{Diff}^{s}(M)
$$

is given as follows: for $\xi \in \mathfrak{X}^{s+1}(M)$ let $\phi_{t} \in \operatorname{Diff}^{s}(M)$ be its flow. Then the curve $\phi(t)=\phi_{t}$ is an integral curve of the right invariant vector field $Y_{\xi}$ on $\operatorname{Diff}^{s}(M)$ through $e=\operatorname{id}_{M}$. Indeed, $\phi(0)=\phi_{0}=\operatorname{id}_{M}$, and for $x \in$ $M, \dot{\phi}(t)(x)=\left.\frac{\mathrm{d} \phi(t)(x)}{d t}\right|_{t=0}=\left.\frac{\mathrm{d} \phi_{t}(x)}{d t}\right|_{t=0}=\xi\left(\phi_{t}(x)\right)=(\xi \circ \phi(t))(x)=$ $T R_{\phi_{t}}(x)=Y_{\xi}(\phi(t))(x)$. Hence $\dot{\phi}(t)=Y_{\xi}\left(\phi_{t}\right)$ and $\dot{\phi}(0)=\xi$. Now define

$$
\exp (\xi):=\phi_{1} .
$$

Then $\exp : \mathfrak{X}^{s}(M) \rightarrow \operatorname{Diff}^{s}(M)$ is continuous, but not $C^{1}$. Moreover exp is not locally surjective, i.e. exp does not map onto any neighborhood of the identity in $\operatorname{Diff}^{s}(M)$. This is the reason we cannot use this exponential map to define directly charts on $\operatorname{Diff}^{s}(M)$. In other words, the diffeomorphism group $\mathcal{G}=\operatorname{Diff}^{s}(M)$ is not generated by exponentiating vector fields. In every neighborhood of $\mathrm{id}_{M}$ there are diffeomorphisms which are not part of the flow of any vector field, in short

$$
\operatorname{Diff}^{s}(M) \neq \exp \mathfrak{X}^{s}(M) .
$$

Summarizing we have the following "Lie group" structure of $\operatorname{Diff}^{s}(M)$ :

i) multiplication $\mu: \operatorname{Diff}^{s+k}(M) \times \operatorname{Diff}^{s}(M) \rightarrow \operatorname{Diff}^{s}(M)$, is $C^{k}$

ii) inversion $\nu: \operatorname{Diff}^{s+k}(M) \rightarrow \operatorname{Diff}^{s}(M)$, is $C^{k}$

iii) the Lie bracket for $\xi, \eta \in \mathfrak{X}^{s+1}(M)$ is only $H^{s}$ i.e. $[\xi, \eta] \in \mathfrak{X}^{s}(M)$

iv) the exponential map exp : $\mathfrak{X}^{s+1}(M) \rightarrow \operatorname{Diff}^{s}(M)$ is not locally onto.

This non-classical "Lie group" structure of $\operatorname{Diff}^{s}(M)$ leads to the definition of so called ILH-Lie groups (Inverse Limit of Hilbert).

\subsection{ILH-Lie Groups and ILH-Lie Algebras}

Definition 5. A collection of groups $\left\{G^{\infty}, G^{s} ; s \geq s_{0}\right\}$ is called an ILH-Lie group (Inverse Limit of Hilbert) if: 
i) each $G^{s}$ is a Hilbert manifold of class $C^{k(s)}$ modeled on a Hilbert space $E^{s}$, and $k(s) \rightarrow \infty$ as $s \rightarrow \infty$

ii) for each $s \geq s_{0}$ there are linear continuous, dense inclusions $E^{s+1} \hookrightarrow E^{s}$ and dense inclusions of class $C^{k(s)}: G^{s+1} \hookrightarrow G^{s}$

iii) each $G^{s}$ is a topological group and $G^{\infty}=\underset{s}{\lim } G^{s}$ is a topological group with the inverse limit topology

iv) if $\left(U^{s}, \varphi^{s}, E^{s}\right)$ is a chart of $G^{s}$ then $\left(U^{s} \cap G^{t},\left.\varphi^{s}\right|_{U^{s} \cap G^{t}}, E^{t}\right)$ is a chart for $G^{t}$ for all $t \geq s$

v) the multiplication $\mu: G^{\infty} \times G^{\infty} \rightarrow G^{\infty}$ extends to a $C^{k}$ map $\mu: G^{s+k} \times G^{s} \rightarrow$ $G^{s}$ for all $s$ such that $k \leq k(s)$

vi) the inversion $\nu: G^{\infty} \rightarrow G^{\infty}$ extends to a $C^{k}$ map $\nu: G^{s+k} \rightarrow G^{s}$, for all $s$ such that $k \leq k(s)$

vii) the right multiplication $R_{g}$ by $g \in G^{s}$ extends to a $C^{k(s)}$ map $R_{g}: G^{s} \rightarrow G^{s}$.

Definition 6. A collection of vector spaces $\left\{\mathfrak{g}^{\infty}, \mathfrak{g}^{s} ; s \geq s_{0}\right\}$ is called an ILHLie algebra if:

i) each $\mathfrak{g}^{s}$ is a Hilbert space and for each $s \geq s_{0}$ there are linear, continuous, dense inclusions $\mathfrak{g}^{s+1} \hookrightarrow \mathfrak{g}^{s}$ and $\mathfrak{g}^{\infty}=\underset{s}{\lim } \mathfrak{g}^{s}$ is a Frechet space with the inverse limit topology

ii) there exist bilinear, continuous, antisymmetric maps [, ] : $\mathfrak{g}^{s+2} \times \mathfrak{g}^{t+2} \rightarrow$ $\mathfrak{g}^{\min (s, t)}$, for all $s, t \geq s_{0}$, which satisfy the Jacobi identity on $\mathfrak{g}^{\min (s, t, r)}$ for elements in $\mathfrak{g}^{s+4} \times \mathfrak{g}^{t+4} \times \mathfrak{g}^{r+4}$.

Theorem 7. Let $\left\{G^{\infty}, G^{s} ; s \geq s_{0}\right\}$ be and ILH-Lie group and for each s let $\mathfrak{g}^{s}:=T_{e} G^{s}$ and $\mathfrak{g}^{\infty}=\underset{s}{\lim } \mathfrak{g}^{s}$. Then $\left\{\mathfrak{g}^{\infty}, \mathfrak{g}^{s} ; s \geq s_{0}\right\}$ is the ILH-Lie algebra of the ILH Lie group $\left\{G^{\infty}, G^{s} ; s \geq s_{0}\right\}$.

\section{Example: Diffeomorphism Groups}

$$
\left\{\operatorname{Diff}^{\infty}(M), \operatorname{Diff}^{s}(M) ; s>\frac{1}{2} \operatorname{dim} M\right\}
$$

is an ILH-Lie group with ILH-Lie algebra

$$
\left\{\mathfrak{X}^{\infty}(M), \mathfrak{X}^{s}(M) ; s>\frac{1}{2} \operatorname{dim} M\right\}
$$

where $k(s)=\infty$ for all $s$.

\section{Remarks:}

1) The Nash-Moser implicit function theorem works for ILH! 
2) $C^{k}$-diffeomorphism groups, $0 \leq k<\infty$.

For the group $\operatorname{Diff}^{k}(M)$ of diffeomorphisms of class $C^{k}$ we have a similar situation as in the case $\operatorname{Diff}^{s}(M)$ of $H^{s}$ diffeomorphisms discussed above. Diff ${ }^{k}(M)$ is a Banach manifold and one defines the notion of ILB (Inverse Limit of Banach)Lie groups and ILB-Lie algebras similar to ILH. Then $\left\{\operatorname{Diff}^{\infty}(M), \operatorname{Diff}^{k}(M)\right.$; $k \in \mathbb{N}\}$ is an ILB Lie group with ILB-Lie algebra $\left\{\mathfrak{X}^{\infty}(M), \mathfrak{X}^{k}(M) ; k \in \mathbb{N}\right\}$.

3) $C^{\infty}$-diffeomorphisms.

For the group Diff $\operatorname{Din}^{\infty}(M)$ of diffeomorphisms of class $C^{\infty}$ one can take an entirely different point of view. In the $H^{s}$ or $C^{k}$ case the major role is played by the tower of Hilbert or Banach manifolds $\operatorname{Diff}^{s}(M)$ and $\operatorname{Diff}^{k}(M)$ respectively and $\operatorname{Diff}^{\infty}(M)$ is only considered as limiting topological group. In contrast to this one can study $\operatorname{Diff}^{\infty}(M)$ directly as Frechet manifold and Frechet Lie group. For this to make sense one needs a differential calculus in Frechet spaces. Unfortunately, there are many inequivalent ways to define this concept, and no matter what differential calculus one uses, one is always faced with pathologies in the theory of Frechet manifolds; e.g. the classical inverse function theorem and implicit function theorem do not hold. This point of view of differentiablity is developed in Keller [21], Hamilton [20] and Schmid [34].

\subsection{Gauge Groups}

The diffeomorphism subgroups that arise in gauge theories as gauge groups behave nicely because they are isomorphic to subgroups of loop groups which are not only ILH-Lie groups but actually Hilbert Lie groups.

Let $\pi: P \rightarrow M$ be a principal $G$ bundle with $G$ a finite dimensional Lie group (structure group) acting on $P$ from the right $p \in P, g \in G, p \cdot g \in P$.

The Gauge group $\mathcal{G}$ is the group of gauge transformations defined by

$$
\mathcal{G}=\left\{\phi \in \operatorname{Diff}^{\infty}(P) ; \phi(p \cdot g)=\phi(p) \cdot g \quad \text { and } \quad \pi(\phi(p))=\pi(p)\right\} .
$$

$\mathcal{G}$ is a group under composition, hence a subgroup of the diffeomorphism group $\operatorname{Diff}^{\infty}(P)$. Since a gauge transformation $\phi \in \mathcal{G}$ preserves fibers we can realize each such $\phi \in \mathcal{G}$ via $\phi(p)=p \cdot \tau(p)$ where $\tau: P \rightarrow G$ satisfies $\tau(p \cdot g)=$ $g^{-1} \tau(p) g$, for $p \in P, g \in G$. Let

$$
\operatorname{Gau}(P)=\left\{\tau \in C^{\infty}(P, G) ; \tau(p \cdot g)=g^{-1} \tau(p) g\right\} .
$$

$\operatorname{Gau}(P)$ is a group under pointwise multiplication, hence a subgroup of the loop group $C^{\infty}(P, G)$ (see Section 2.4.3), which extends to a Hilbert Lie group if 
equipped with the $H^{s}$-Sobolev topology. We give $\operatorname{Gau}(P)$ the induced topology and extend it to a Hilbert Lie group denoted by $\operatorname{Gau}^{S}(P)$. Another interpretation is that $\operatorname{Gau}(P)$ is isomorphic to $C^{\infty}(\operatorname{Ad} P)$ the space of sections of the associated vector bundle $\operatorname{Ad}(P)=P \times_{G} G$. Completed in the $H^{s}$ Sobolev topology we get $\operatorname{Gau}^{s}(P) \simeq H^{s}(\operatorname{Ad} P)$.

Let $\mathbf{g}$ denote the Lie algebra of $G$. Then the Lie algebra $\mathfrak{g a u}(P)$ of $G a u(P)$ is a subalgebra of the loop algebra $H^{S}(P, \mathbf{g})$ under pointwise bracket in $\mathbf{g}$, the finite dimensional the Lie algebra of $G$; i.e. for any $\xi, \eta \in H^{s}(P, \mathbf{g})$ the bracket is defined by $[\xi, \eta]_{\mathfrak{g a u}(P)}(p)=[\xi(p), \eta(p)]_{\mathfrak{g}}, p \in P$. Then $\mathfrak{g a u}^{s}(P)$ is the subalgebra of Ad-invariant g-valued functions on $P$, i.e.

$$
\mathfrak{g a \mathfrak { u }}(P)=\left\{\xi \in C^{\infty}(P, \mathbf{g}) ; \xi(p \cdot g)=\operatorname{Ad}_{g^{-1}} \xi(p)\right\}
$$

The Lie algebra lie $\mathcal{G}$ (running out of symbols) of the gauge group $\mathcal{G}$ is the Lie subalgebra of $\mathfrak{X}^{\infty}(P)$ consisting of all $G$-invariant vertical vector fields $X$ on $P$, i.e.

$$
\text { lie } \mathcal{G}=\left\{X \in \mathfrak{X}^{\infty}(P) ; R_{g}^{*} X=X, \quad X(p) \in \mathbf{g}, \quad g \in G, \quad p \in P\right\}
$$

with commutator bracket $\left[X_{1}, X_{2}\right]=X_{1} X_{2}-X_{2} X_{1} \in \mathfrak{l i k} \mathcal{G}$.

On the other hand, the Lie algebra of $C^{\infty}(\mathrm{Ad} P)$ is $C^{\infty}(\operatorname{ad}(P))$ - the space of sections of the associated vector bundle ad $(P) \equiv\left(P \times_{G} \mathbf{g}\right) \rightarrow M$ with pointwise bracket.

We have three versions of gauge groups $\mathcal{G}, \operatorname{Gau}(P)$ and $C^{\infty}(\operatorname{Ad} P)$. They are all group isomorphic. There is a natural group isomorphism $\operatorname{Gau}(P) \rightarrow \mathcal{G}: \tau \mapsto \phi$ defined by $\phi(p)=p \cdot \tau(p), p \in P$ which preserves the product $\tau_{1} \cdot \tau_{2} \mapsto \phi_{1} \circ$ $\phi_{2}$. Identifying $\mathcal{G}$ with $\operatorname{Gau}(P)$ we can avoid the troubles with diffeomorphism groups and we can extend $\mathcal{G}$ to a Hilbert Lie group $\mathcal{G}^{s}$. So $\mathcal{G}^{s}$ is actually a Hilbert Lie group in the classical sense, i.e. the group operations are $C^{\infty}$. Also the three Lie algebras lie $\mathcal{G}, \mathfrak{g a u}(P)$ and $C^{\infty}($ ad $P)$ are canonically isomorphic. Indeed, for $s \in C^{\infty}(\operatorname{ad} P)$ define $\xi \in \mathfrak{g a u}(P) \xi: P \rightarrow g$ by $\xi(p \cdot a):=\operatorname{Ad}_{a^{-1}} \xi(p)$; and for $\xi \in \mathfrak{g a u}(P)$ define $s \in C^{\infty}(\operatorname{ad} P)$ by $s(\pi(p)):=[p, \xi(p)]$.

On the other hand, for $\xi \in \mathfrak{g a \mathfrak { u }}(P)$ define $Z_{\xi} \in \mathfrak{l i \mathfrak { e }} \mathcal{G}$ by

$$
Z_{\xi}(p)=\left.\frac{\mathrm{d} R(p, \exp t \xi(p))}{\mathrm{d} t}\right|_{t=0}, \quad\left(=\xi(p)^{*}(p)\right)
$$

i.e. $Z_{\xi}$ is the fundamental vector field on $P$, generated by $\xi \in$ g. $Z_{\xi}$ is invariant iff $\xi(p \cdot g)=\operatorname{Ad}_{g^{-1}} \xi(p)$. 
To topologize lie $\mathcal{G}$ we complete $C^{\infty}($ ad $P)$ in the $H^{s}$-Sobolev norm. If $s>$ $\frac{1}{2} \operatorname{dim} M$ then $\mathfrak{l i e} \mathcal{G}^{s} \simeq H^{s}(\operatorname{ad} P) \simeq \mathfrak{g a n}^{s}(P)$ are isomorphic Hilbert Lie algebras.

There is a natural exponential map Exp : $\mathfrak{g a u}(P) \rightarrow G a u(P)$, which is a local diffeomorphism. Let $\exp : \mathbf{g} \rightarrow G$ be the finite dimensional exponential map. Then define

$$
\operatorname{Exp}: \mathfrak{g a u}^{s}(P) \rightarrow \operatorname{Gau}^{s}(P):(\operatorname{Exp} \xi)(p)=\exp (\xi(p)), \quad \xi \in \mathfrak{g a u ^ { s }}(P) .
$$

Or in terms of $\mathcal{G}, \operatorname{Exp}: \mathfrak{l i} \mathfrak{e} \mathcal{G}^{s} \rightarrow \mathcal{G}^{s}:(\operatorname{Exp} \xi)(p)=p \cdot \exp \left(\xi_{p}\right)$.

Proposition 8. For $s>\frac{1}{2} \operatorname{dim} M$

$$
\mathcal{G}^{s} \simeq \operatorname{Gau}^{s}(P) \simeq H^{s}(\operatorname{Ad} P)
$$

is a smooth Hilbert Lie group with Lie algebra

$$
\mathfrak{l i e} \mathcal{G}^{s} \simeq \mathfrak{g a u}^{s}(P) \simeq H^{s}(\operatorname{ad} P)
$$

and smooth exponential map, which is a local diffeomorphism

$$
\operatorname{Exp}: \operatorname{li} \mathfrak{e} \mathcal{G}^{s} \rightarrow \mathcal{G}^{s}:(\operatorname{Exp} \xi)(p)=p \cdot \exp (\xi(p)) .
$$

\section{Subgroups of Diffeomorphism Groups and Applications}

\subsection{Volume Preserving Diffeomorphisms and Fluid Dynamics}

In this section we show that the Euler equations of fluid dynamics are equivalent to the geodesic equations on the group of volume preserving diffeomorphisms as well as existence and uniqueness of solutions [13].

Let $M$ be a compact manifold, $\operatorname{dim} M=n$ and $\mu$ a volume form on $M$ i.e. $\mu$ is a nondegenerate $n$-forms with $\mu(x) \neq 0, \forall x \in M$. Let

$$
\operatorname{Diff}_{\mu}^{s}(M)=\left\{f \in \operatorname{Diff}^{s}(M) ; f^{*} \mu=\mu\right\}
$$

be the set of volume preserving $H^{s}$ diffeomorphisms on $M$. Since the pull back satisfies $(f \circ g)^{*}=g^{*} \circ f^{*}$ it follows that $\operatorname{Diff}_{\mu}^{s}(M) \subset \operatorname{Diff}^{s}(M)$ is a subgroup. Is it a LIE SUBGROUP in the sense of ILH-Lie groups?

Let's first look for a candidate for the corresponding Lie algebra, i.e. the tangent space at the identity $T_{e} \operatorname{Diff}_{\mu}^{s}(M)$. Let $\xi \in \mathfrak{X}^{s}(M)$ with flow $\varphi_{t} \in \operatorname{Diff}_{\mu}^{s}(M)$. 
Since $\varphi_{t}^{*} \mu=\mu$ it follows that $0=\left.\frac{\mathrm{d} \varphi_{t}^{*}}{\mathrm{~d} t}\right|_{t=0}=L_{\xi} \mu$, the Lie derivative of $\mu$ along $\xi$. Recall that the divergence $\operatorname{div} \xi$ of a vector field $\xi$ is defined by $L_{\xi} \mu=(\operatorname{div} \xi) \mu$, so $L_{\xi} \mu=0 \Leftrightarrow \operatorname{div} \xi=0$ and we get

$$
T_{e} \operatorname{Diff}_{\mu}^{s}(M)=\mathfrak{X}_{\mu}^{s}(M)=\left\{\xi \in \mathfrak{X}^{s}(M) ; \operatorname{div} \xi=0\right\}
$$

the space of divergence free (incompressible) vector fields on $M$. Since $L_{\xi \xi, \eta]}=$ $\left[L_{\xi}, L_{\eta}\right]$ we conclude that $\mathfrak{X}_{\mu}^{s}(M) \subset \mathfrak{X}^{s}(M)$ is a Lie subalgebra.

How do we show that $\operatorname{Diff}_{\mu}^{s}(M)$ is a Lie subgroup of $\operatorname{Diff}^{s}(M)$ ? Recall in the classical (finite dimensional) case we have the following fundamental facts:

- For any finite dimensional Lie algebra $\mathrm{g}$ there is a finite dimensional Lie group $G$ for which $\mathbf{g}$ is the Lie algebra, i.e. $\mathbf{g} \simeq T_{e} G$. This is false in infinite dimensions!

- Any closed subgroup of a finite dimensional Lie group is a Lie subgroup. This is false in infinite dimension!

Nevertheless the following was shown by Ebin and Marsden [13]

Theorem 9. $\operatorname{Diff}_{\mu}^{s}(M)$ is a closed smooth submanifold of $\operatorname{Diff}^{s}(M)$.

Proof: (idea) The difficulty is to prove that $\operatorname{Diff}_{\mu}^{s}(M)$ is a smooth manifold. Let us illustrate the proof that $\operatorname{Diff}_{\mu}^{s}(M)$ is a closed submanifold of $\operatorname{Diff}^{s}(M)$. This proof is typical in the theory of diffeomorphism groups, so we outline the basic ideas, which are simple and are based on two key facts:

Let $\Omega^{n}(M)$ denote the space of $n$-forms on $M$ and consider the map

$$
F_{\mu}: \operatorname{Diff}^{s}(M) \rightarrow \Omega^{n}(M): F_{\mu}(f)=f^{*} \mu .
$$

Then one shows that for a small enough subspace $[\mu]_{s}$ of $\Omega^{n}(M)$ this map is a $C^{\infty}$ submersion. Then for fixed $\mu \in[\mu]_{s}$ we have $\operatorname{Diff}_{\mu}^{s}(M)=F^{-1}(\mu)$, i.e. $\operatorname{Diff}_{\mu}^{s}(M)$ is the inverse se image of one point under a submersion, hence a closed submanifold and "Lie subgroup".

The first step uses the Hodge decomposition theorem to ensure that the affine space $[\mu]_{s}:=\mu+\mathrm{d}\left(H^{s+1}\left(\Omega^{n-1}(M)\right)\right.$ is a closed subspace in $H^{s}\left(\Omega^{n}(M)\right)$. We denote by $H^{s}\left(\Omega^{k}(M)\right)$ the space of exterior $k$-forms on $M$ of Sobolev class $H^{s}$. Define the map $F_{\mu}: \operatorname{Diff}^{s}(M) \rightarrow[\mu]_{s}, \quad F_{\mu}(f):=f^{*}(\mu)$. Then $F_{\mu}$ is well defined, i.e. $F_{\mu}(f) \in[\mu]_{s}$ is in the right space. Indeed by the change of variable theorem we have $\int_{M}\left(f^{*} \mu-\mu\right)=0$, so that by the DeRham theorem we get $f^{*} \mu-\mu=\mathrm{d} \alpha$ for some $\alpha \in H^{s-1}\left(\Omega^{n-1}(M)\right)$. 
The second step uses the $\Omega$-lemma to prove that $F_{\mu}$ is a $C^{\infty}$ submersion. The derivative of $F_{\mu}$ is obtained as

$$
T_{f} F_{\mu}\left(\xi_{f}\right)=f^{*}\left(L_{\xi} \mu\right), \quad \xi_{f} \in T_{f} \operatorname{Diff}^{s}(M)
$$

where $\xi=\xi_{f} \circ f^{-1} \in \mathfrak{X}^{s}(M)$. Hence $F_{\mu}$ is $C^{\infty}$. For $f=e=\operatorname{id}_{M}: T_{e} F_{\mu}(\xi)=$ $L_{\xi} \mu=\mathrm{d} i_{\xi} \mu+i_{\xi} \mathrm{d} \mu$ but since $\mu$ is a volume element on $M, \mathrm{~d} \mu=0$. Moreover $\mu$ is nondegenerate, hence the map

$$
\xi \in \mathfrak{X}_{\mu}^{s}(M) \mapsto i_{\xi} \mu \in H^{s}\left(\Omega^{n-1}\right)
$$

is an isomorphism ( $i_{\xi}$ denotes the inner product). Therefore $T_{e} F_{\mu}$ is onto, hence $F_{\mu}$ is a submersion and $F_{\mu}^{-1}(\mu)=\operatorname{Diff}_{\mu}^{s}(M)$ is a closed submanifold of $\operatorname{Diff}^{s}(M)$. Note that this proof gives the charts on $\operatorname{Diffs}_{\mu}^{s}(M)$ only implicitly. Omori [28] showed the following

Theorem 10. $\left\{\operatorname{Diff}_{\mu}^{\infty}(M), \operatorname{Diff}_{\mu}^{s}(M) ; s>\frac{1}{2} \operatorname{dim} M\right\}$ is an ILH Lie group with $\left\{\mathfrak{X}_{\mu}^{\infty}(M), \mathfrak{X}_{\mu}^{s}(M) ; s>\frac{1}{2} \operatorname{dim} M\right\}$ its ILH Lie algebra.

Application to Fluid Dynamics: The main application of $\operatorname{Diff}_{\mu}^{s}(M)$ is based on the fact that it is the configuration space of incompressible, homogeneous, ideal fluids. Let $M$ be a compact orientable, finite dimensional Riemannian manifold and let $\mu$ be the Riemannian volume. Then $\operatorname{Diff}_{\mu}^{s}(M)$ admits the smooth weak Riemannian metric as follows: For $m \in M$ let $<,>_{m}$ be the inner product on $T_{m} M$. Let $\eta \in \operatorname{Diff}_{\mu}^{s}(M)$ and $X, Y \in T_{\eta} \operatorname{Diff}_{\mu}^{s}(M)$. Then $X(m)$ and $Y(m)$ are in $T_{\eta(m)} M$. The inner product on each tangent space $T_{\eta} \operatorname{Diff}_{\mu}^{s}(M)$ is defined as

$$
(X, Y)_{\eta}=\int_{M}<X(m), Y(m)>_{\eta(m)} \mathrm{d} \mu(m) \quad X, Y \in T_{\eta} \operatorname{Diff}_{\mu}^{s}(M) .
$$

With this weak Riemannian metric on $\operatorname{Diff}_{\mu}^{s}(M)$ we can talk about geodesics on $\operatorname{Diff}_{\mu}^{s}(M)$. It turns out that this metric is right invariant and that its spray is smooth. This remarkable fact found by Ebin and Marsden [13] has as consequence the local existence and uniqueness of geodesics on $\operatorname{Diff}_{\mu}^{s}(M)$ and their smooth dependence on initial conditions. Moreover, if $V_{t}$ is an integral curve of the spray on $\operatorname{Diff}_{\mu}^{s}(M)$ and $\eta_{t}=\tau_{M} \circ V_{t}$ i.e. $\dot{\eta}_{t}=V_{t}$ then

$$
v_{t}:=V_{t} \circ \eta_{t}^{-1}
$$

satisfies the Euler equations:

$$
\frac{\partial v}{\partial t}+\nabla_{v} v=-\nabla p, \quad \operatorname{div} v=0
$$


where $\nabla_{v}$ is the covariant derivative of the metric on $M$. The time dependent vector field $v_{t}$ on $M$ represents the Eulerian (or spatial) velocity of the fluid, whereas the solution $V_{t}$ of the geodesic spray equation represents the material velocity of the fluid. Incompressibility of the flow implies that $\eta_{t} \in \operatorname{Diff}_{\mu}^{s}(M)$. In this way, the well-posedness of the Euler equations is equivalent to the local existence and uniqueness of solutions for the geodesic spray on $\operatorname{Diff}_{\mu}^{s}(M)$. The dependence of the solution of the Euler equations on initial conditions is continuous, because the pull back $v_{t}=V_{t} \circ \eta_{t}^{-1}$ involves right composition with an inverse as well as left translation and both operations are continuous but not smooth.

Theorem 11. (Ebin-Marsden [13])

- $v$ satisfies the Euler equations $\Leftrightarrow \eta_{t}$ is a geodesic on $\operatorname{Diff}_{\mu}^{s}(M)$

- existence of $C^{\infty}$ geodesics on $\operatorname{Diff}_{\mu}^{s}(M)$ for small $t$

- the Euler equations has unique solution for small $t$, depending $C^{\infty}$ on the initial condition $v_{0}$.

The relationship $v_{t}=V_{t} \circ \eta_{t}^{-1}$ represents the momentum map of the right action of the group $\operatorname{Diff}_{\mu}^{s}(M)$ on the weak symplectic manifold $T \operatorname{Diff}_{\mu}^{s}(M)$, the symplectic form being induced naturally by the weak metric from the canonical symplectic structure on the cotangent bundle $T^{*} \operatorname{Diff}_{\mu}^{s}(M)$.

We (Eichhorn and Schmid [15]) proved the same theorem for the topological Euler equation on open (non compact) manifolds (see last chapter).

\subsection{Canonical Transformations (Symplectomorphisms) and Plasma Physics}

In this section we show that the Maxwell-Vlasov equations of plasma physics are an infinite dimensional Hamiltons system on a space involving the diffeomorphism subgroup of canonical transformations.

Let $M$ be a compact manifold, $\operatorname{dim} M=2 n$, and $\omega$ be a symplectic structure $M$, i.e. $\omega$ is a nondegenerate closed $(\mathrm{d} \omega=0)$ 2-form on $M$. Consider

$$
\operatorname{Diff}_{\omega}^{s}(M)=\left\{f \in \operatorname{Diff}^{s}(M) ; f^{*} \omega=\omega\right\}
$$

the group of symplectomorphisms, or canonical transformations, on $M$. Using similar arguments as for volume preserving diffeomorphisms it is shown that

Theorem 12. $\left\{\operatorname{Diff}_{\omega}^{\infty}(M), \operatorname{Diff}_{\omega}^{s}(M) ; s>n\right\}$ is an ILH-Lie subgroup of the ILH-Lie group $\left\{\operatorname{Diff}^{\infty}(M), \operatorname{Diff}^{s}(M) ; s>n\right\}$, with ILH-Lie algebra $\left\{\mathfrak{X}_{\omega}^{\infty}(M)\right.$, $\left.\mathfrak{X}_{\omega}^{s}(M) ; s>n\right\}$, where $\mathfrak{X}_{\omega}^{s}(M)=\left\{\xi \in \mathfrak{X}^{s}(M) ; L_{\xi} \omega=0\right\}$ is the Lie algebra of locally Hamiltonian $H^{s}$-vector fields. 
See Ebin-Marsden [13] and Omori [28] for details. Again the charts on Diffs $\omega_{\omega}(M)$ are obtain only implicitly. However, in this case, there is a direct method due to Weinstein [43] to construct explicit charts using Poincare's generating functions. In Schmid [33], [35] it is shown directly using the so called $\Gamma$-differentiability for Frechet spaces, that $\operatorname{Diff}_{\omega}^{\infty}(M)$ is a Frechet manifold and Frechet Lie group with $\Gamma$-differentiable group operations.

\subsubsection{Application to Plasma Physics}

A plasma is a collection of charged particles of various species (electrons, protons, ect.) moving in $\mathbb{R}^{3}$ as a "charged fluid". For simplicity one assumes that there is only one species of particles of charge $q$ and mass $m$, and it is useful to approximate their positions $x \in \mathbb{R}^{3}$ and velocities $v$ by a density function on phase space which may be a smooth function $f(x, v, t)$. Denote by $E(x, t)$ and $B(x, t)$ the electric and magnetic fields respectively generated by the motion of the charged particles. Then the plasma can be described by the Maxwell-Vlasov equations

$(M V)\left\{\begin{array}{l}\frac{\partial f}{\partial t}+v \cdot \frac{\partial f}{\partial x}+\frac{q}{m}(E+v \times B) \frac{\partial f}{\partial v}=0 \quad \text { (Boltzman equation) } \\ \frac{\partial B}{\partial t}=-\operatorname{curl} E \\ \frac{\partial E}{\partial t}=\operatorname{curl} B-J_{f}, \text { where the current density } J_{f}=q \int v f(x, v, t) \mathrm{d} v \\ \operatorname{div} E=\rho_{f}, \text { where the charge density } \rho_{f}=q \int f(x, v, t) \mathrm{d} v \\ \operatorname{div} B=0 .\end{array}\right.$

We think of this system of coupled, non-linear system of evolution equations as an initial value problem for the variables $f, E$ and $B$. The following was shown by Marsden and Weinstein in 1982, see [25] for a summary.

Theorem 13. The Maxwell-Vlasov equations are an infinite dimensional Hamiltonian system, i.e. they can be written in the form

$$
\dot{F}=\{F, H\}
$$

for a certain non-canonical Poisson bracket $\{$,$\} and some Hamiltonian H$. 
Let us outline the ideas of the construction of this Hamiltonian structure, see [25] and [35] for details.

\subsubsection{The Poisson-Vlasov System}

We first consider the limit case where $B=0$. Then the Maxwell-Vlasov system reduces to a single equation of the field variable $f(x, v, t)$ the Poisson-Vlasov equation

$$
\frac{\partial f}{\partial t}+v \cdot \frac{\partial f}{\partial v}-\frac{q}{m} \frac{\partial \phi_{f}}{\partial x} \cdot \frac{\partial f}{\partial x}=0
$$

where the scalar potential $\phi_{f}$ is given by $\Delta \phi_{f}=-\rho_{f}$. One can show that $f(x, v, t)$ evolves in time by a canonical transformation $\eta$ of $\mathbb{R}^{6}$, i.e. $f(x, v, t)=$ $\eta_{t}^{*} f\left(x, v, t_{0}\right)$ where $\eta_{t} \in \operatorname{Diff}_{\omega}\left(\mathbb{R}^{6}\right)$ and $\omega$ is the canonical symplectic form on $\mathbb{R}^{6}$. If we identify any Hamiltonian vector field $X_{h}(x, v)$ on $\mathbb{R}^{6}$ with its Hamiltonian function $h(x, v): \mathbb{R}^{6} \rightarrow \mathbb{R}$, we get a Lie algebra isomorphism $\mathfrak{g}=\mathfrak{X}_{\omega}^{\infty}\left(\mathbb{R}^{6}\right) \cong C^{\infty}\left(\mathbb{R}^{6}\right)$ with $\left[X_{h}, X_{g}\right]=X_{\{h, g\}}$, where $\{h, g\}$ is the canonical Poisson bracket of functions on $\mathbb{R}^{6}$. Moreover, if we identify the dual of this Lie algebra $\mathfrak{g}^{*}$ via the $L^{2}$-pairing $\left\langle h, f>=\int h(x, v) f(x, v) \mathrm{d} x \mathrm{~d} v\right.$ with itself $\mathfrak{g}$, we can regard the plasma density as $f(x, v) \in \mathfrak{g}^{*} \cong C^{\infty}\left(\mathbb{R}^{6}\right)$.

On the dual $\mathfrak{g}^{*}$ of any Lie algebra $\mathfrak{g}$ we have the Lie Poisson bracket for functions $F, G: \mathfrak{g}^{*} \rightarrow \mathbb{R}$, given by the formula

$$
\{F, G\}(\mu)=\left\langle\mu,\left[\frac{\delta F}{\delta \mu}, \frac{\delta G}{\delta \mu}\right]\right\rangle, \quad \mu \in \mathfrak{g}^{*}
$$

where $\frac{\delta F}{\delta \mu}, \frac{\delta G}{\delta \mu}$ are regarded as elements of $\mathfrak{g}$ and $[$,$] denotes the Lie bracket in$ $\mathfrak{g}$ and $\langle$,$\rangle the canonical pairing between \mathfrak{g}^{*}$ and $\mathfrak{g}$.

Now a direct computation shows that the Poisson-Vlasov equation (46) is in LiePoisson form on $\mathfrak{g}^{*}=C^{\infty}\left(\mathbb{R}^{6}\right)$ i.e. $\dot{F}=\{F, H\}$ with energy

$$
H(f)=\frac{1}{2} \int m v^{2} f(x, v, t) \mathrm{d} x \mathrm{~d} v+\frac{1}{2} \int \phi_{f} \rho_{f} \mathrm{~d} x
$$

and $\{$,$\} the Lie-Poisson bracket on \mathfrak{g}^{*}=C^{\infty}\left(\mathbb{R}^{6}\right)$ given by

$$
\{F, G\}(f)=\int f\left\{\frac{\delta F}{\delta f}, \frac{\delta G}{\delta f}\right\} \mathrm{d} x \mathrm{~d} v .
$$




\subsubsection{The Maxwell Equations}

As we discussed in Section 2.4.2, Maxwell's equations

$$
\dot{E}=\operatorname{curl} B, \quad \dot{B}=-\operatorname{curl} E
$$

are canonical Hamilton's equations on $T^{*} \mathcal{A}$ with respect to the canonical symplectic structure and the Hamiltonian $H(E, B)=\frac{1}{2} \int\left(|E|^{2}+|B|^{2}\right) \mathrm{d} x$ is the total field energy. Here the configuration space $\mathcal{A}=\left\{A: \mathbb{R}^{3} \rightarrow \mathbb{R}^{3}\right\}$ is the space of vector potentials on $\mathbb{R}^{3}$ and $B=\operatorname{curl} A$ for some $A \in \mathcal{A}$. Then we consider $(A, E) \in T^{*} \mathcal{A}$. The invariance of Maxwell's equations under the gauge transformations $A \mapsto A+\nabla \phi$ for any $\phi \in C^{\infty}\left(\mathbb{R}^{3}\right)$ leads via the reduction procedure to the two remaining Maxwell equations

$$
\operatorname{div} E=\rho, \operatorname{div} B=0 .
$$

\subsubsection{The Coupled System: The Maxwell-Vlasov Equations}

Combining the Poisson-Vlasov system (46) and the Maxwell equations (50), (51) we get the Maxwell-Vlasov system. The same symmetry group $C^{\infty}\left(\mathbb{R}^{6}\right)$ that leaves Maxwell's equations invariant, acts on the coupled phase space $T_{\text {id }} \operatorname{Diff}_{\omega}^{\infty}\left(\mathbb{R}^{6}\right) \times T^{*} \mathcal{A} \simeq C^{\infty}\left(\mathbb{R}^{6}\right) \times T^{*} \mathcal{A}$. Reducing by this symmetry, one obtains a reduced phase space with a Poisson structure with respect to which the Maxwell-Vlasov equations are a Hamiltonian system, i.e in the form

$$
\dot{F}=\{F, H\}
$$

with the Hamiltonian

$$
H(f, E, B)=\frac{1}{2} \int m v^{2} f(x, v) \mathrm{d} x \mathrm{~d} v+\frac{1}{2} \int|B(x)|^{2} \mathrm{~d} x+\frac{1}{2} \int|E(x)|^{2} \mathrm{~d} x
$$

which is the total energy of the plasma. The noncanonical Poisson bracket on the reduced phase space turns out to be the following: For any two functions $F$ and $G$ of the field variables $(f, E, B)$ we have

$$
\begin{aligned}
&\{F, G\}(f, E, B)=\int f\left\{\frac{\delta F}{\delta f}, \frac{\delta G}{\delta f}\right\} \mathrm{d} x \mathrm{~d} v \\
&+\int\left(\frac{\delta F}{\delta E} \cdot \operatorname{curl} \frac{\delta G}{\delta B}-\frac{\delta G}{\delta E} \cdot \operatorname{curl} \frac{\delta F}{\delta B}\right) \mathrm{d} x+\int\left(\frac{\delta F}{\delta E} \cdot \frac{\partial f}{\partial v} \frac{\delta G}{\delta f}-\frac{\delta G}{\delta E} \cdot \frac{\partial f}{\partial v} \frac{\delta F}{\delta f}\right) \mathrm{d} x \mathrm{~d} v \\
&+\int f B \cdot\left(\frac{\partial}{\partial v} \frac{\delta F}{\delta f} \times \frac{\partial}{\partial v} \frac{\delta G}{\delta f}\right) \mathrm{d} x \mathrm{~d} v
\end{aligned}
$$


With this Poisson bracket and Hamiltonian (53) the Maxwell-Vlasov equations are an infinite dimensional Hamiltonian system of the form

$$
\dot{F}=\{F, H\} \text {. }
$$

Notice that the coupling of the plasma and electromagnetic fields appears in the Poisson structure rather then in the Hamiltonian, and it is produced by the action of the infinite dimensional gauge group $C^{\infty}\left(\mathbb{R}^{6}\right)$ on the uncoupled phase space of matter and fields.

In this example where a dynamical system of infinitely many degrees of freedom (the Maxwell-Vlasov equations) is described as a Hamiltonian system, infinite dimensional Lie groups appear as a configuration space as well as symmetry groups. Similar structures were found for multifluid plasma, see [25] and references therein.

\subsection{Contact Transformations on $\dot{T}^{*} M$}

This example of a diffeomorphism group plays an important role in our next chapter, where we'll discuss the group of invertible Fourier integral operators.

Let $M$ be a compact manifold, $\operatorname{dim} M=n$ and $T^{*} M$ its cotangent bundle with the canonical symplectic structure $\omega=\mathrm{d} \theta=\sum \mathrm{d} p_{i} \wedge \mathrm{d} q_{i}$ (locally), where $\theta=\sum p_{i} \mathrm{~d} q_{i}$ is the canonical 1-form on $T^{*} M$. We want to study diffeomorphisms $\varphi: T^{*} M \rightarrow T^{*} M$ that preserve $\theta$, i.e $\varphi^{*} \theta=\theta$. Such a diffeomorphism is necessarily a lift, i.e. of the form $\varphi=T^{*} \eta$ for some $\eta \in \operatorname{Diff}^{s}(M)$ i.e. $\varphi$ is just an extended point transformation. To avoid this trivial situation, we delete the zero section in $T^{*} M$ and we consider $\dot{T}^{*} M:=T^{*} M \backslash\{0\}$. Then $\varphi^{*} \theta=\theta \Leftrightarrow$ $\varphi^{*} \omega=\omega$ and $\varphi\left(\tau \alpha_{x}\right)=\tau \varphi\left(\alpha_{x}\right)$ for all $\tau>0, \alpha_{x} \in T_{x}^{*} M$ i.e. $\varphi^{*} \theta=\theta \Leftrightarrow \varphi$ is symplectic and homogeneous of degree one. Consider

$$
\operatorname{Diff}_{\theta}^{s}\left(\dot{T}^{*} M\right)=\left\{\varphi \in \operatorname{Diff}^{s}\left(\dot{T}^{*} M\right) ; f^{*} \theta=\theta\right\}
$$

the group of $H^{s}$ contact transformations on $\dot{T}^{*} M$. Note that $\dot{T}^{*} M$ is not compact, so we cannot apply our previous constructions to establish $\operatorname{Diffs}_{\theta}^{s}\left(\dot{T}^{*} M\right)$ as an ILH Lie group. Nevertheless we (Ratiu and Schmid [32]) showed the following

Theorem 14. $\left\{\operatorname{Diff}_{\theta}^{\infty}\left(\dot{T}^{*} M\right), \operatorname{Diff}_{\theta}^{s}\left(\dot{T}^{*} M\right) ; s>\operatorname{dim} M+1\right\}$ is an ILH Lie group.

Sketch of proof: The main problem is that $\dot{T}^{*} M$ is not compact, so our previous methods do not apply to the full diffeomorphism group Diff ${ }^{s}\left(\dot{T}^{*} M\right)$. Therefore, 
the subgroup $\operatorname{Diff}_{\theta}^{s}\left(\dot{T}^{*} M\right)$ of $\operatorname{Diff}^{s}\left(\dot{T}^{*} M\right)$ cannot inherit a submanifold structure from $\operatorname{Diff}^{s}\left(\dot{T}^{*} M\right)$ as described in Section 4.1.

The main idea is to "make" $\dot{T}^{*} M$ compact, that means to pass to the cosphere bundle of $M$ and show that $\operatorname{Diff}_{\theta}^{s}\left(\dot{T}^{*} M\right)$ is algebraically isomorphic to the group of contact $H^{s}$-transformations (quantomorphisms) on the cosphere bundle.

\subsubsection{The Cosphere Bundle $S\left(T^{*} M\right)$}

The multiplicative group $\mathbb{R}_{+}$acts smoothly on $\dot{T}^{*} M$ by $\alpha_{x} \mapsto \tau \alpha_{x}, \tau \in \mathbb{R}_{+}$, $\alpha_{x} \in \dot{T}_{x}^{*} M$. This action is free and proper, hence the orbit space $S\left(T^{*} M\right):=$ $\left(\dot{T}^{*} M\right) / \mathbb{R}_{+}$is a smooth manifold, called the cosphere bundle of $M$. Note that $S\left(T^{*} M\right)$ is compact if $M$ is compact and $\operatorname{dim} S\left(T^{*} M\right)=2 n-1$. Moreover, the canonical projection $\pi: \dot{T}^{*} M \rightarrow S\left(T^{*} M\right)$ defines a smooth principal fiber bundle with structure group $\mathbb{R}_{+}$. There is no canonical contact structure on $S\left(T^{*} M\right)$, i.e. no one form $\tilde{\theta}$ such that $\pi^{*} \tilde{\theta}=\theta$, but one can construct a whole family of contact structures by use of global sections $\sigma: S\left(T^{*} M\right) \rightarrow \dot{T}^{*} M, \pi \circ \sigma=\mathrm{id}$.

Given such a global section $\sigma$, define $\theta_{\sigma}:=\sigma^{*} \theta$. Then $\theta_{\sigma}$ is an exact contact 1 -form on $S\left(T^{*} M\right)$, i.e. $\theta_{\sigma} \wedge\left(\theta_{\sigma}\right)^{n-1}$ is a volume form on $S\left(T^{*} M\right)$. Note that $\pi^{*} \theta_{\sigma} \neq \theta$ but $\sigma$ is uniquely determined by a function $f_{\sigma}: \dot{T}^{*} M \rightarrow \mathbb{R}$ defined by $\sigma\left(\pi\left(\alpha_{x}\right)\right)=f_{\sigma}\left(\alpha_{x}\right) \alpha_{x}$; i.e. $f_{\sigma}$ measures the distance from the section $\sigma$. Then

$$
\pi^{*} \theta_{\sigma}=f_{\sigma} \cdot \theta
$$

\subsubsection{Contact Transformations on $S\left(T^{*} M\right)$}

Let $\eta \in \operatorname{Diff}_{\theta}^{s}\left(\dot{T}^{*} M\right)$ and $\sigma: S\left(T^{*} M\right) \rightarrow \dot{T}^{*} M$ is a fixed section. Since $\eta^{*} \theta=\theta$, $\eta$ is homogeneous degree +1 , there is a unique diffeomorphism $\varphi: S\left(T^{*} M\right) \rightarrow$ $S\left(T^{*} M\right)$ defined by $\varphi \circ \pi=\pi \circ \eta$. Write $\varphi=\pi \circ \frac{1}{f_{\sigma}} \eta \circ \sigma$, indeed for $\alpha_{x} \in$ $\dot{T}^{*} M, \varphi\left(\pi\left(\alpha_{x}\right)\right)=\pi\left(\frac{1}{f_{\sigma}} \eta\left(\sigma\left(\pi\left(\alpha_{x}\right)\right)\right)=\pi\left(\frac{1}{f_{\sigma}} \eta\left(f_{\sigma}\left(a_{x}\right) \cdot \alpha_{x}\right)=\pi\left(\eta\left(\alpha_{x}\right)\right)\right.\right.$. Compute $\varphi^{*} \theta_{\sigma}=\sigma^{*} \frac{1}{f_{\sigma}} \eta^{*} \pi^{*} \theta_{\sigma}=\sigma^{*} \frac{1}{f_{\sigma}} \eta^{*}\left(f_{\sigma} \cdot \theta\right)=\sigma^{*}\left(f_{\sigma} \circ \frac{\eta}{f_{\sigma}}\right) \cdot \eta^{*} \theta=$ $\sigma^{*}\left(f_{\sigma} \circ \frac{\eta}{f_{\sigma}}\right) \cdot \sigma^{*} \theta=\left(f_{\sigma} \circ \frac{\eta}{f_{\sigma}} \circ \sigma\right) \theta_{\sigma}=h_{\sigma} \cdot \theta_{\sigma}$ where $h_{\sigma}:=f_{\sigma} \circ \frac{\eta}{f_{\sigma}} \circ \sigma$.

This means that the pair $\left(\varphi, h_{\sigma}\right)$ is a contact transformation for $\theta_{\sigma}$ i.e. $\varphi^{*} \theta_{\sigma}=$ $h_{\sigma} \theta_{\sigma}$, where $h_{\sigma}: S\left(T^{*} M\right) \rightarrow \mathbb{R}_{+}$.

So, to each $\eta$ with $\eta^{*} \theta=\theta$, there corresponds a unique contact transformation $(\varphi, h)$ with $\varphi^{*} \theta_{\sigma}=h \theta_{\sigma}$. Vice versa, for a pair $(\varphi, h)$ with $\varphi^{*} \theta_{\sigma}=h \theta_{\sigma}$ we define 
$\eta$ by $\eta=(\sigma \circ \varphi \circ \pi) /(h \circ \pi) f_{\sigma}$. Then $\eta^{*} \theta=\theta$ and the maps $\eta \mapsto(\varphi, h)$ and $(\varphi, h) \mapsto \eta$ are inverses of each other.

The composition corresponds to the semidirect product in the following way: let $\eta_{1} \leftrightarrow\left(\varphi_{1}, h_{1}\right)$ and $\eta_{2} \leftrightarrow\left(\varphi_{2}, h_{2}\right)$, then $\eta_{1} \circ \eta_{2} \leftrightarrow\left(\varphi_{1}, h_{1}\right) \cdot\left(\varphi_{2}, h_{2}\right)=\left(\varphi_{1} \circ\right.$ $\left.\varphi_{2}, h_{2} \cdot\left(h_{1} \circ \varphi_{2}\right)\right)$. The above map is a group isomorphism of $\operatorname{Diff}_{\theta}^{s}\left(\dot{T}^{*} M\right)$ with the semidirect product $\operatorname{Diff}^{s} S\left(T^{*} M\right) \ltimes C^{s}\left(S\left(T^{*} M\right), \dot{\mathbb{R}}\right)$, where $C^{s}\left(S\left(T^{*} M\right), \dot{\mathbb{R}}\right)$ is regarded as a multiplicative group.

Theorem 15. (Ratiu-Schmid[32])

1) The group of contact transformations

$$
\begin{aligned}
\operatorname{Con}_{\sigma}^{s}\left(S\left(T^{*} M\right)\right) & := \\
& \left\{(\varphi, h) \in \operatorname{Diff}^{s} S\left(T^{*} M\right) \ltimes C^{s}\left(S\left(T^{*} M\right), \dot{\mathbb{R}}\right) ; \varphi^{*} \theta_{\sigma}=h \theta_{\sigma}\right\}
\end{aligned}
$$

is a closed $H^{s}$ Lie subgroup of the semidirect product

$\operatorname{Diff}^{s} S\left(T^{*} M\right) \bowtie C^{s}\left(S\left(T^{*} M\right), \dot{\mathbb{R}}\right)$.

2) $\operatorname{Diff}_{\theta}^{s}\left(\dot{T}^{*} M\right)$ is isomorphic (as group) to $\operatorname{Con}_{\sigma}^{s}\left(S\left(T^{*} M\right)\right)$.

Remarks: 1) Since the cosphere bundle $S\left(T^{*} M\right)$ is compact, one can apply the standard methods to show that $\operatorname{Con}^{s}\left(S\left(T^{*} M\right)\right)$ is a closed submanifold of $\operatorname{Diff}^{s} S\left(T^{*} M\right) \ltimes C^{s}\left(S\left(T^{*} M\right)\right.$.

2) For two different sections $\sigma$ and $\tau$ we have an isomorphism $\operatorname{Con}_{\sigma}^{s}\left(S\left(T^{*} M\right)\right) \simeq$ $\operatorname{Con}_{\tau}^{s}\left(S\left(T^{*} M\right)\right)$, hence the manifold structure on $\operatorname{Diff}_{\theta}^{s}\left(\dot{T}^{*} M\right)$ is independent of the choice of the section $\sigma$.

\subsubsection{The Corresponding Lie Algebras}

The Lie algebra of $\operatorname{Diff}^{s} S\left(T^{*} M\right) \ltimes C^{s}\left(S\left(T^{*} M\right), \dot{\mathbb{R}}\right)$ is the semidirect product $\mathfrak{X}^{s}\left(S\left(T^{*} M\right)\right) \ltimes C^{s}\left(S\left(T^{*} M\right), \mathbb{R}\right)$ with induced bracket

$$
[(X, f),(Y, g)]=([X, Y], X(g)-Y(f)) .
$$

The Lie algebra of $\operatorname{Con}_{\sigma}^{s}\left(S\left(T^{*} M\right)\right)$ is

$$
\mathfrak{c o n}_{\sigma}^{s}\left(S\left(T^{*} M\right)\right)=\left\{(Y, g) \in \mathfrak{X}^{s}\left(S\left(T^{*} M\right)\right) \ltimes C^{s}\left(S\left(T^{*} M\right), \mathbb{R}\right) ; L_{Y} \theta_{\sigma}=g \theta_{\sigma}\right\}
$$

the space of infinitesimal contact transformations.

On the other hand, the Lie algebra of $\operatorname{Diff}_{\theta}^{s}\left(\dot{T}^{*} M\right)$ is $\mathfrak{X}_{\theta}^{s}\left(S\left(T^{*} M\right)\right)=\{Y \in$ $\left.\mathfrak{X}^{s}\left(S\left(T^{*} M\right)\right) ; L_{Y} \theta=0\right\}$. Now $L_{Y} \theta=0 \Leftrightarrow Y$ is a globally Hamiltonian vector 
field, homogeneous of degree 0 , i.e. $Y=Y_{H}$ and $H$ is homogeneous of degree 1 . $H$ is determined uniquely by $H=\theta(Y)$.

Let $C_{\text {hom }}^{s}\left(\dot{T}^{*} M\right)=\left\{H \in C^{s}\left(T^{*} M, \mathbb{R}\right) ; H\right.$ homogeneous degree 1$\}$. $C_{h o m}^{s}\left(\dot{T}^{*} M\right)$ is a Lie algebra with canonical Poisson bracket

$$
\{F, H\}=\sum \frac{\partial F}{\partial q_{i}} \frac{\partial H}{\partial p_{i}}-\frac{\partial H}{\partial q_{i}} \frac{\partial F}{\partial p_{i}}
$$

and is isomorphic to the Lie algebra $\mathfrak{X}_{\theta}^{s}\left(\dot{T}^{*} M\right)$ with the commutator bracket $[X, Y]=X Y-Y X$.

Theorem 16. (Ratiu-Schmid [32]) The groups $\operatorname{Diff}_{\theta}^{s}\left(\dot{T}^{*} M\right)$ and $\operatorname{Con}_{\sigma}^{s}\left(S\left(T^{*} M\right)\right)$ are isomorphic as ILH-Lie groups with isomorphic ILH-Lie algebras $\mathfrak{X}_{\theta}^{s}\left(\dot{T}^{*} M\right) \cong$ $\operatorname{con}_{\sigma}^{s}\left(S\left(T^{*} M\right)\right) \cong C_{h o m}^{s}\left(\dot{T}^{*} M\right)$.

Remarks: 1) We will see in the next section that elements of $\operatorname{Diff}_{\theta}^{s}\left(\dot{T}^{*} M\right)$ represent phase functions of Fourier integral operators and elements of $C_{\mathrm{hom}}^{s}\left(\dot{T}^{*} M\right)$ are interpreted as symbols of pseudodifferential operators.

2) These spaces also play a role in quantization.

\subsection{Globally Hamiltonian Vector Fields}

There is another interesting subgroup of the diffeomorphism group Diff ${ }_{\omega}^{s}(M)$ whose Lie algebra consists of all globally Hamiltonian vector fields.

Let $(M, \omega)$ be a compact symplectic manifold and denote by $\mathcal{H}^{\infty}(M)$ the space of globally Hamitonian vector fields on $M$, i.e. $X \in \mathcal{H}^{\infty}(M)$ iff $X=X_{H}$ with $H$ : $M \rightarrow \mathbb{R}$ and $\omega\left(X_{H}, Y\right)=\mathrm{d} H \cdot Y$ or equivalently $i_{X_{H}} \omega=\mathrm{d} H$. We have the relation

$$
X_{\{F, H\}}=\left[X_{H}, X_{F}\right]
$$

hence $\mathcal{H}^{\infty}(M)$ is a Lie subalgebra of $\mathfrak{X}^{\infty}(M)$. The question is: Is there a corresponding Lie group?

We have seen that $\operatorname{Diff}_{\omega}^{s}(M)$ is an ILH-Lie subgroup of $\operatorname{Diff}^{s}(M)$ with ILH Lie algebra $\mathfrak{X}_{\omega}^{\infty}(M)$ the locally Hamiltonian vector fields. It was shown by Calabi [9] and Arnold [4] that the commutator algebra $\left[\mathfrak{X}_{\omega}^{\infty}(M), \mathfrak{X}_{\omega}^{\infty}(M)\right]=\mathcal{H}^{\infty}(M)$ is the Lie algebra of globally Hamiltonian vector fields.

Recall: If $\mathbf{g}, \mathbf{h}$ are Lie algebras, then their commutator algebra $[\mathbf{g}, \mathbf{h}]$ is generated by $[X, Y]$ with $X \in \mathbf{g}, Y \in \mathbf{h}$. If $G, H$ are Lie groups, then their commutator group $[G, H]$ is generated by $g h g^{-1} h^{-1}$ with $g \in G, h \in H$.

Let $\operatorname{Diff}_{\omega}^{\infty}(M)_{0}$ denote the identity component of $\operatorname{Diff}_{\omega}^{\infty}(M)$. 
Theorem 17. (Ratiu-Schmid [32]) The commutator subgroup $\left[\operatorname{Diff}_{\omega}^{\infty}(M)_{0}\right.$, $\left.\operatorname{Diff}_{\omega}^{\infty}(M)_{0}\right]$ is a simple, closed ILH-Lie subgroup of $\operatorname{Diff}_{\omega}^{\infty}(M)_{0}$ with ILH Lie algebra

$$
\mathcal{H}^{\infty}(M)=\left[\mathfrak{X}_{\omega}^{\infty}(M), \mathfrak{X}_{\omega}^{\infty}(M)\right] .
$$

Proof: (for details see Banyaga [5] and Calabi [9]) Consider the map

$$
S: \operatorname{Diff}_{\omega}^{s+1}(M)_{0} \rightarrow H^{1}(M, \mathbb{R}), S(h)=\left[A\left(h_{t}\right)\right]
$$

where $h_{t}$ is a symplectic homotopy from $h$ to id ${ }_{M}$ with the locally Hamiltonian vector field $X_{t}=\frac{\mathrm{d} h_{t}}{\mathrm{~d} t} \circ h_{t}^{-1}$. Define $A\left(h_{t}\right)=\int_{0}^{1} i\left(X_{t}\right) \omega \mathrm{d} t$ which is a closed $H^{s+1}$-one form defining a cocycle in $H^{1}(M, \mathbb{R})$. One shows the following:

- $S$ is a group homomorphism and $\operatorname{Ker} S$ is perfect. $H^{1}(M, \mathbb{R})$ is abelian, therefore $\operatorname{Ker} S=\left[\operatorname{Diff}_{\omega}^{\infty}(M)_{0}, \operatorname{Diff}_{\omega}^{\infty}(M)_{0}\right]$.

- $S$ is a $C^{\infty}$ submersion, therefore $\operatorname{Ker} S$ is a closed $H^{s+1}$-Lie subgroup of $\operatorname{Diff}_{\omega}^{s+1}(M)_{0}$.

\subsection{The Group of Quantomorphisms}

Let $(M, \theta)$ be a compact exact contact manifold, i.e. $M$ is smooth $\operatorname{dim} M=$ $(2 n+1)$ and $\theta$ is a 1 -form such that $\theta \wedge(\mathrm{d} \theta)^{n}$ is a volume element on $M$. Consider

$$
\operatorname{Diff}_{\theta}^{s}(M)=\left\{\eta \in \operatorname{Diff}^{s}(M) ; \eta^{*} \theta=\theta\right\}
$$

called the quantomorphism group of $M$.

The characteristic bundle of $\mathrm{d} \theta$ is given by $R_{\mathrm{d} \theta}=\left\{v \in T M ; i_{v} \mathrm{~d} \theta=0\right\}$. It is integrable and one-dimensional. The Reeb vector field $E$ of $R_{\mathrm{d} \theta}$ is the unique section of the line bundle $R_{\mathrm{d} \theta}$ defined by $i_{E} \theta=1$ and $i_{E} \mathrm{~d} \theta=0$. In local coordinates $\left(x^{1}, \ldots, x^{n}, y^{1}, \ldots, y^{n}, t\right)$, we have $\theta=\sum_{i=1}^{n} y^{i} \mathrm{~d} x^{i}+\mathrm{d} t$ and $E=$ $\frac{\partial}{\partial t}$. Let $R_{\theta}=\{v \in T M ; \theta(v)=0\}$ be the characteristic bundle of $\theta$. Then $\operatorname{dim} R_{\theta}=2 n$ and $T M=R_{\mathrm{d} \theta} \oplus R_{\theta}$, hence $\mathfrak{X}^{s}(M)=\mathfrak{X}^{s}\left(R_{\mathrm{d} \theta}\right) \oplus \mathfrak{X}^{s}\left(R_{\theta}\right)$.

The leaves of the foliation $\mathcal{F}$ defined by the line bundle $R_{\mathrm{d} \theta}$ (circle action) are the integral curves of $E$. Then the quotient manifold $N=M / \mathcal{F}$ is a smooth manifold which carries a symplectic structure $\omega$ such that the projection $\pi: M \rightarrow N$ satisfies $\pi^{*} \omega=\mathrm{d} \theta$. So, $\theta$ becomes the connection 1-form on this principal circle bundle whose horizontal subbundle is $R_{\theta}$ and $\omega$ is its curvature 2-form. Thus $M$ is the quantizing manifold of $N$ whose automorphism group $\operatorname{Diff}_{\theta}^{s}(M)=\{\eta \in$ $\left.\operatorname{Diff}^{s}(M) ; \eta^{*} \theta=\theta\right\}$ is called the quantomorphism group of $(M, \theta)$. 
Let $\mathcal{K}^{s}(N)=\left\{\varphi \in \operatorname{Diff}_{\omega}^{s}(N) ; H_{\varphi \circ \gamma}=H_{\gamma}=\right.$ horizontal transport along $\left.\gamma\right\}$. We have the following reformulation of Kostant's prequantization theorem

Theorem 18. (Kostant [22]) The following sequence of groups is exact

$$
0 \longrightarrow S^{1} \stackrel{J}{\longrightarrow} \operatorname{Diff}_{\theta}^{s}(M) \stackrel{P}{\longrightarrow} \mathcal{K}^{s}(N) \longrightarrow 0
$$

and the following sequence of Lie algebras is exact

$$
0 \longrightarrow \mathbb{R} \stackrel{j}{\longrightarrow} \mathfrak{X}_{\theta}^{s}(M) \stackrel{p}{\longrightarrow} \mathcal{H}^{s}(N) \longrightarrow 0 .
$$

We showed that this is an exact sequence of ILH-Lie groups with corresponding ILH-Lie algebras i.e.

Theorem 19. (Ratiu-Schmid [32]) The quantomorphism group $\operatorname{Diff}_{\theta}^{\infty}(M)$ is an ILH principal circle bundle over the ILH-Lie group $\mathcal{K}^{\infty}(N)$ with ILH Lie algebra $\mathfrak{X}_{\theta}^{s}(M)=\left\{X \in \mathfrak{X}^{\infty}(M) ; L_{X} \theta=0\right\}$ of infinitesimal quantomorphisms.

\subsection{The Group of Gauge Transformations and Quantum Field Theory}

The diffeomorphism subgroups that arise in gauge theories as gauge groups behave nicely because they are isomorphic to subgroups of loop groups as discussed in Section 3.5 .

Consider a principal $G$-bundle $\pi: P \rightarrow M$ and the group $\mathcal{G}$ of gauge transformations

$$
\begin{aligned}
\mathcal{G} & =\left\{\phi \in \operatorname{Diff}^{\infty}(P) ; \phi(p \cdot g)=\phi(p) \cdot g, \pi \phi(p)=\pi(p)\right\} \\
& \cong\left\{\tau \in C^{\infty}(P, G) ; \tau(p \cdot g)=g^{-1} \tau(p) g\right\}=\operatorname{Gau}(P)
\end{aligned}
$$

which is a smooth Hilbert Lie group with smooth group operations (see Section 3.5). We only sketch here what role this infinite dimensional gauge group $\mathcal{G}$ plays in these quantum field theories. A good reference for this topics is P. Deligne et al. [12]. Let $\mathcal{A}$ denote the space of connection 1 -forms on $P$ (vector potentials). Each $A \in \mathcal{A}$ defines a covariant differential $D_{A}$ and a curvature 2-form (field strength) $F_{A}=D_{A} A=\mathrm{d} A+\frac{1}{2}[A, A]$. They are locally given by $A=A_{\mu} \mathrm{d} x^{\mu}$ and $F=\frac{1}{2} F_{\mu \nu} \mathrm{d} x^{\mu} \wedge \mathrm{d} x^{\nu}$ where $F_{\mu \nu}=\partial_{\mu} A_{\nu}-\partial_{\nu} A_{\mu}+\left[A_{\mu}, A_{\nu}\right]$.

The gauge group $\mathcal{G}$ acts on $\mathcal{A}$ via pull-back $\phi \in \mathcal{G}, A \in \mathcal{A}, \phi \cdot A=\left(\phi^{-1}\right)^{*} A \in \mathcal{A}$, or under the isomorphism (see Section 3.5) $\mathcal{G} \cong \operatorname{Gau}(P), \phi \Leftrightarrow \tau$ we have 
$\operatorname{Gau}(P)$ acting on $\mathcal{A}$ by $\tau \cdot A=\tau A \tau^{-1}+\tau \mathrm{d} \tau^{-1}$. Hence the covariant derivative transforms as $D_{\tau \cdot A}=\tau D_{A} \tau^{-1}$ and the action on the field is $\tau \cdot F_{A}:=F_{\tau} \cdot A=$ $\tau F_{A} \tau^{-1}$. The action functional (Yang-Mills functional) is $S(A)=\left\|F_{A}\right\|^{2}$, locally given by $\left\|F_{A}\right\|^{2}=\frac{1}{2} \int_{M} \operatorname{trace}\left(F_{\mu \nu} F^{\mu \nu}\right)$. This action is gauge invariant $S(\phi \cdot A)=S(A), \phi \in \mathcal{G}$, so the Yang-Mills functional is defined on the orbit space $\mathcal{M}=\mathcal{A} / \mathcal{G}$. The space $\mathcal{M}$ is in general not a manifold since the action of $\mathcal{G}$ on $\mathcal{A}$ is not free. If we restrict to irreducible connections then $\mathcal{M}$ is a smooth infinite dimensional manifold and $\mathcal{A} \rightarrow \mathcal{M}$ is an infinite dimensional principal fiber bundle with structure group $\mathcal{G}$. For self-dual connections $F_{A}=* F_{A}$ (instantons) on a compact 4-manifold the moduli space

$$
\mathcal{M}=\{A \in \mathcal{A} ; A \text { self-dual }\} / \mathcal{G}
$$

is a smooth finite dimensional manifold.

Self-dual connections absolutely minimize the Yang-Mills action integral

$$
\mathbf{Y M}(A)=\int_{\Omega}\left\|F_{A}\right\|^{2}, \quad \Omega \subset M \text { compact. }
$$

The Feynman path integral quantizes the action and we get the probability amplitude

$$
W(f)=\int_{\mathcal{A} / \mathcal{G}} e^{-S(A)} f(A) \mathcal{D}(A)
$$

for any gauge invariant functional $f(A)$.

\section{BRST symmetry}

In 1976 C. Bechi, A. Rouet and R. Stora [6] (and independently I. Tyutin in 1975) discovered that in gauge field theories the effective Lagrangian, which is no longer gauge invariant, is still invariant under a new class of transformations, now called BRST transformations $\mathfrak{s}$, given by

$$
\mathfrak{s} A=d \eta+[A, \eta], \mathfrak{s} \eta=-\frac{1}{2}[\eta, \eta]
$$

where $A$ is the potential field (connection one form) and $\eta$ is the ghost field. An important property of the BRST charge $\mathfrak{s}$ is its nilpotency $\mathfrak{s}^{2}=0$. We give an interpretation of these BRST transformation in terms of the Lie algebra cohomology of the Lie group of gauge transformations. 


\subsection{Quantum Chromo Dynamics (QCD) and Quantum Electro Dynamics (QED)}

In classical field theory one considers a Lagrangian $\mathcal{L}\left(\phi_{i}, \partial_{\mu} \phi_{i}\right)$ of the fields $\phi_{i}$ : $\mathbb{R}^{n} \rightarrow \mathbb{R}, i=1, \ldots k, \partial_{\mu}=\frac{\partial}{\partial x_{\mu}}$ and the corresponding action functional $S=$ $\int \mathcal{L}\left(\phi_{i}, \partial_{\mu} \phi_{i}\right) \mathrm{d}^{n} x$. The variational principle $\delta S=0$ then leads to the EulerLagrange equations of motion

$$
\frac{\partial \mathcal{L}}{\partial \phi_{i}}-\partial_{\mu} \frac{\partial \mathcal{L}}{\partial\left(\partial_{\mu} \phi_{i}\right)}=0
$$

In QED and QCE the Lagrangian is more complicated, of the form $\mathcal{L}(A, \psi, \varphi)=$

$$
-\frac{1}{4 g^{2}} \operatorname{trace} F_{\mu \nu} F^{\mu \nu}-i \bar{\psi}\left[\gamma^{\mu}\left(\partial_{\mu}+i e A_{\mu}\right)+m\right] \psi+\left(D_{A}^{\mu} \varphi\right)^{\dagger}\left(D_{A}^{\mu} \varphi\right)-m^{2} \varphi^{\dagger} \varphi
$$

where $A_{\mu}(x)$ is a potential 1 -form (boson) and the field strength $F$ is given by $F_{\mu \nu}=\partial_{\mu} A_{\nu}-\partial_{\nu} A_{\mu}+\left[A_{\mu}, A_{\nu}\right]$. In QED the gauge group of the principal bundle is $G=U(1)$, and in QCD we have $G=S U(2)$. The Dirac $\gamma$-matrices are $\gamma^{i}=\left(\begin{array}{cc}0 & -\sigma_{i} \\ \sigma_{i} & 0\end{array}\right)$ where $\sigma_{i}$ are the Pauli matrices (canonical basis of $\mathfrak{s u}(2)$ ) and $\bar{\psi}=\psi^{\dagger} \gamma^{o}$ is the Pauli adjoint with $\gamma^{o}=\left(\begin{array}{ll}0 & 1 \\ 1 & 0\end{array}\right), m$ is the electron mass, $e$ the electron charge and $g$ a coupling constant.

\subsubsection{The Equations of Motion}

The variational principle of the Lagrangian (64) with respect to the fields $A, \psi$ and $\varphi$ gives the corresponding Euler Lagrange equations of motion. They describe for instance the motion of an electron $\psi(x)$ (fermion, spinor) in an electromagnetic field $F$, interacting with a bosonic field $\varphi$. We get from the variational principle $\frac{\delta S}{\delta A_{\mu}}=0 \Rightarrow \partial_{\mu} F^{\mu \nu}=e \bar{\psi} \gamma^{\nu} \psi$, which are Maxwell's equations for $G=U(1)$. In the free case i.e., when $\psi=0$ we get $\partial_{\mu} F^{\mu \nu}=0$, the vacuum Maxwell equations.

For $G=S U(2)$ these equations become $D^{\mu} F_{\mu \nu}=0$, the Yang-Mills equations. $\frac{\delta S}{\delta \psi}=0 \Rightarrow \mathrm{i}\left(\partial_{A}-m\right) \psi=0$, which are Dirac's equations, where $\not_{A}=\gamma^{\mu}\left(\partial_{\mu}+\right.$ 
ie $\left.A_{\mu}\right)=\gamma^{\mu} D_{A}^{\mu}$. In the free case i.e. when $A=0$ we get $\mathrm{i}(\not \partial-m) \psi=0$, the classical Dirac equation.

\subsubsection{Global Formulation}

Consider a principal $G$-bundle $\pi: P \rightarrow M$, with $M$ a compact, orientable Riemannian manifold (e.g. $M=S^{4}, T^{4}$ ) and $G$ a compact nonabelian gauge group with Lie algebra $\mathrm{g}$. Let $\mathcal{A}$ be the infinite dimensional affine space of connection 1 -forms on $P$. So each $A \in \mathcal{A}$ is a g-valued, equivariant 1-form on $P$ (also called vector potential) and defines the covariant derivative of any field $\varphi$ by $D_{A} \varphi=\mathrm{d} \varphi+\frac{1}{2}[A, \varphi]$. The curvature 2-form $F_{A}$ (or field strength) is a $\mathbf{g}$ valued 2-form and is defined as $F_{A}=D_{A} A=\mathrm{d} A+\frac{1}{2}[A, A]$.

In pure Yang-Mills theory the action functional is given by

$$
S(A)=\frac{1}{2}\left\|F_{A}\right\|^{2}=\frac{1}{2} \int_{M} \operatorname{trace}\left(F_{\mu \nu} F^{\mu \nu}\right)
$$

and the Yang-Mills equations become globally

$$
\mathrm{d} * F_{A}=0 .
$$

With added fermionic field $\psi$ interaction the action becomes

$$
S(A, \psi)=\frac{1}{2}\left\|F_{A}\right\|^{2}+<\partial_{A} \psi, \psi>
$$

where $\psi$ is a section of the spin bundle $\operatorname{Spin}^{ \pm}(M)$ and $\not_{A}: \operatorname{Spin}^{ \pm}(M) \rightarrow$ $\operatorname{Spin}^{\mp}(M)$ is the induced Dirac operator.

\subsection{Symmetries}

\subsubsection{Gauge Invariance}

Let $\mathcal{G}$ be the group of gauge transformations as introduced in Sections 3.5 and 4.6. So $\phi \in \mathcal{G} \Leftrightarrow \phi: P \rightarrow P$ is a diffeomorphism over id ${ }_{M}$, i.e. $\phi(p \cdot g)=\phi(p) \cdot g$, $p \in P, g \in G$. Then $\mathcal{G}$ acts on $\mathcal{A}$ and $\operatorname{Spin}^{ \pm}(M)$ by $\phi \cdot A=\left(\phi^{-1}\right)^{*} A$, and $\phi \cdot \psi=\left(\phi^{-1}\right)^{*} \psi$. The action functionals $S$ are gauge invariant:

$$
\begin{gathered}
\text { Yang-Mills: } \quad S(\phi \cdot A)=S(A), \quad A \in \mathcal{A}, \quad \phi \in \mathcal{G} . \\
\text { QED : } \quad S(\phi \cdot A, \phi \cdot \psi)=S(A, \psi), \quad A \in \mathcal{A}, \quad \psi \in \operatorname{Spin}^{ \pm}(M), \phi \in \mathcal{G}
\end{gathered}
$$




\subsubsection{Chiral Symmetry}

The chiral symmetry is the symmetry that leads to anomalies and the BRST invariance. In QCD the chiral symmetry of the Fermi field $\psi$ is given by

$$
\psi \mapsto \mathrm{e}^{\mathrm{i} \beta \gamma_{5}} \psi
$$

where $\beta$ is a constant and $\gamma_{5}=\mathrm{i} \gamma^{o} \gamma^{1} \gamma^{2} \gamma^{3}$. The classical Noether current of this symmetry is given by

$$
J_{\mu}=\bar{\psi} \gamma_{\mu} \gamma_{5} \psi
$$

which is conserved, i.e. $\partial^{\mu} J_{\mu}=0$.

This conservation law breaks down after quantization and one gets

$$
\partial^{\mu} J_{\mu}=2 \mathrm{i} m \bar{\psi} \gamma_{5} \psi-\frac{g^{2}}{8 \pi^{2}} \operatorname{trace} F_{\mu \nu} F^{\mu \nu} \equiv \omega \neq 0
$$

This value $\omega$ is called the chiral anomaly.

\subsection{Quanization}

The quantization is given by the Feynman path integral:

$$
\int_{\mathcal{A} / \mathcal{G} \times \text { Spin }} \int \mathrm{e}^{\mathrm{i} S(A, \psi)} \mathcal{F}(A, \psi) \mathcal{D} A \mathcal{D} \psi=\langle\mathcal{F}(A, \psi)\rangle
$$

which computes the expectation value $\langle\mathcal{F}(A, \psi)\rangle$ of the function $\mathcal{F}(A, \psi)$. This in an integral over two infinite dimensional spaces, the gauge orbit space $\mathcal{A} / \mathcal{G}$ and the fermionic Berezin integral over the spin-space $\operatorname{Spin}^{ \pm}(M)$. These integrals are mathematically not defined but physicist compute them by gauge fixing, i.e. fixing a section $\sigma: \mathcal{A} / \mathcal{G} \longrightarrow \mathcal{A}$, (e.g. $\sigma(A)=\partial_{\mu} A^{\mu}=0$, the Lorentz gauge) and then integrate over the section $\sigma$. Such a section does not exist globally, but only locally (Gribov ambiguity!). The effect of such a gauge fixing is that one gets extra terms in the Lagrangian (gauge fixing terms) and one has to introduce new fields, so called ghost fields $\eta$ via the Faddeev-Popov procedure. The such obtained effective Lagrangian is no longer gauge invariant. This effective Lagrangian has the form in QCD:

$$
\begin{array}{rrr}
\mathcal{L}_{\text {eff }}(A, \psi, \eta)= & \frac{1}{2} \operatorname{trace}\left(F_{\mu \nu} F^{\mu \nu}\right) & \text { kinetic energy } \\
& +\frac{1}{2 \alpha} \operatorname{trace}\left(\partial_{\mu} A^{\mu}\right)^{2} & \text { gauge fixing term } \\
& -g \partial_{\mu} \bar{\eta} D_{A}^{\mu} \eta & \text { ghost term } \\
& +\ldots \ldots & \text { interaction terms }
\end{array}
$$


We can write this globally as

$$
\mathcal{L}_{\mathrm{eff}}=\frac{1}{2}\left\|F_{A}\right\|^{2}+\frac{1}{2}\|\sigma(A)\|^{2}+\bar{\eta} \mathcal{M} \eta+\ldots
$$

where $\mathcal{M}=\frac{\delta}{\delta \phi}(\sigma(\phi \cdot A))$ is the Faddeev-Popov determinant, acting like the Jacobian of the global gauge variation $\frac{\delta}{\delta \phi}$ over the section $\sigma$. Writing this term in the exponent of the action functional like a "fermionic Gaussian integral" leads to the Faddeev-Popov ghost fields $\eta, \bar{\eta}$ in the form $\operatorname{det} \mathcal{M}=\int e^{-\bar{\eta} \mathcal{M} \eta} \mathrm{d} \bar{\eta} \mathrm{d} \eta$.

The effective Lagrangian $\mathcal{L}_{\text {eff }}$ is NOT gauge invariant but has a new symmetry, called BRST symmetry.

\subsection{BRST Symmetries}

Named after Becchi, Rouet and Stora [6] and Tyutin [41] who discovered this invariance in 1975 and 1976 the BRST operator $\mathfrak{s}$ is given as follows

$$
\left.\begin{array}{l}
\mathfrak{s} A=\mathrm{d} \eta+[A, \eta] \\
\mathfrak{s} \eta=-\frac{1}{2}[\eta, \eta]
\end{array}\right\} \quad \mathcal{L}_{\mathrm{eff}} \text { is BRST invariant. }
$$

Note that the BRST operator $\mathfrak{s}$ mixes bosons and fermions and it is nilpotent i.e. $\mathfrak{s}^{2}=0$. The question arises whether this operator $\mathfrak{s}$ is the coboundary operator of some kind of cohomology. The affirmative answer is given by the following theorem:

Theorem 20. (Schmid [36] and [38]) Let $\mathcal{C}^{q, p}\left(\mathfrak{l i e} \mathcal{G}, \Omega_{\mathrm{loc}}\right)$ be the ChevalleyEilenberg complex of the Lie algebra lie $\mathcal{G}$ of infinitesimal gauge transformations, with respect to the induced adjoint representation on local forms $\Omega_{\mathrm{oc}}$, with boundary operator

$$
\delta_{\mathrm{loc}}: \mathcal{C}^{q, p}\left(\mathfrak{l i e} \mathcal{G}, \Omega_{\mathrm{loc}}\right) \longrightarrow \mathcal{C}^{q+1, p}\left(\mathfrak{l i e} \mathcal{G}, \Omega_{\mathrm{loc}}\right), \quad \delta_{\mathrm{loc}}^{2}=0 .
$$

Then with $\mathfrak{s}:=\frac{(-1)^{p+1}}{q+1} \delta_{\text {loc }}$ we have $\mathfrak{s}^{2}=0$ and the following:

1) for $q=0, p=1, A \in \mathcal{A} \subset \mathcal{C}^{0,1}$, then $\mathfrak{s} A=\mathrm{d} \eta+[A, \eta]$

2) for $q=1, p=0, \eta \in \mathcal{C}^{1,0}$, then $\mathfrak{s} \eta=-\frac{1}{2}[\eta, \eta]$ the Maurer Cartan form

$3)$ the chiral anomaly $\omega$ (given by equ. (72)) is represented as cohomology class of this complex $[\omega] \in \mathcal{H}_{B R S T}^{1,0}\left(\mathfrak{l i c}_{\mathcal{G}} \mathcal{G}, \Omega_{\mathrm{loc}}\right)$. 


\subsubsection{The Chevalley-Eilenberg Cohomology}

We are now going to explain the previous theorem, in particular the general definition of the Chevalley-Eilenberg [11] complex and the corresponding cohomology. Let $G$ be a Lie group with Lie algebra $\mathbf{g}$ and let $\sigma$ be a representation of $\mathbf{g}$ on the vector space $W$. Denote by $\mathcal{C}^{q}(\mathbf{g}, \mathbf{W})$ the space of $W$-valued $q$-cochains on $\mathbf{g}$ and define the coboundary operator $\delta: \mathcal{C}^{q}(\mathbf{g}, \mathbf{W}) \longrightarrow \mathcal{C}^{\mathbf{q}+\mathbf{1}}(\mathbf{g}, \mathbf{W})$ by

$$
\begin{aligned}
\delta \Phi\left(\xi_{o}, \cdots, \xi_{q}\right)=\sum_{i=0}^{q}( & -1)^{i} \sigma\left(\xi_{i}\right) \Phi\left(\xi_{o}, \cdots, \hat{\xi}_{i}, \cdots, \xi_{q}\right) \\
& +\sum_{i<j}(-1)^{i+j} \Phi\left(\sigma\left(\xi_{i}\right) \xi_{j}, \cdots, \hat{\xi}_{i}, \cdots, \hat{\xi}_{j}, \cdots, \xi_{q}\right)
\end{aligned}
$$

We have $\delta^{2}=0$ and define the Lie algebra cohomology of $\mathbf{g}$ with respect to $(\sigma, W)$ as $\mathcal{H}^{*}(\mathbf{g}, \mathbf{W})=\operatorname{ker}(\delta) / \operatorname{im}(\delta)$. This is called the Chevalley-Eilenberg cohomology [11] of the Lie algebra $\mathbf{g}$ with respect to the representation $\sigma$.

\subsubsection{Local Differential Forms}

Local forms $\Omega_{\text {loc }}$ are defined as follows in terms of the jet bundle $J^{\infty}(\pi)$ of the associated vector bundle $\pi$. Consider the space of exterior forms on $M \times \Gamma^{\infty}$, i.e. $\Omega\left(M \times \Gamma^{\infty}(\pi)\right)=\bigotimes_{p, q} \Omega^{p, q}\left(M \times \Gamma^{\infty}(\pi)\right)$ which has a bigradation induced from the product $M \times \Gamma^{\infty}(\pi)$. A form $\alpha \in \Omega^{p, q}\left(M \times \Gamma^{\infty}(\pi)\right)$ is called local iff for any section $s \in \Gamma^{\infty}(\pi)$, and vector fields $X_{1} \cdots X_{q}$ on $J^{\infty}(\pi)$, the induced $p$-form $\alpha\left(s, X_{1} \cdots X_{q}\right)$ on $M$, defined by

$$
\alpha\left(s, X_{1} \cdots X_{q}\right)(x) \equiv\left(i_{X_{1}(s)} \cdots i_{X_{q}(s)} \alpha\right)(x, s)
$$

depends on $s, X_{1}(s), \cdots, X_{q}(s)$ in a local fashion, i.e. $\alpha\left(s, X_{1} \cdots X_{q}\right)(x)$ depends only on finite jets (i.e. finite derivatives) of $s, X_{1}(s), \cdots, X_{q}(s)$ at $x$. In local coordinates a local form $\alpha$ looks like:

$$
\alpha=\sum_{i, j} \alpha_{i_{1} \cdots i_{p} j_{1} \cdots j_{q}} \mathrm{~d} x_{i_{1}} \wedge \cdots \wedge \mathrm{d} x_{i_{p}} \wedge \partial u_{j_{1}} \wedge \cdots \wedge \partial u_{j_{q}}
$$

where the $\alpha_{i_{1} \cdots i_{p} j_{1} \cdots j_{q}}$ are local $(0,0)$ forms, the $\mathrm{d} x_{i}{ }^{\prime} s$ are local $(1,0)$ forms and the $\partial u_{j}{ }^{\prime} s$ are local $(0,1)$ forms. 


\subsubsection{Special Case: The BRST-Cohomology}

In our case for the BRST symmetry we have the following special case of the Chevalley-Eilenberg construction: The Lie group is $G=\mathcal{G}$ the infinite dimensional Lie group of gauge transformations and the Lie algebra is $\mathfrak{g}=$ lie $\mathcal{G}$ the infinite dimensional Lie algebra of infinitesimal gauge transformations and $W=\bigoplus_{p} \Omega_{\text {loc }}^{p}(P, \mathbf{g})$ the space of Lie algebra valued ad-equivariant local $p$-forms on $P$ with $\sigma$ the representation of $\mathfrak{g}$ on $W$ induced by the adjoint representation. We have the double complex $\mathcal{C}^{q, p} \equiv \mathcal{C}^{q}\left(\mathfrak{l i}_{\mathfrak{e}} \mathcal{G}, \Omega_{\text {loc }}^{p}(P, \mathbf{g})\right)$ with coboundary operator $\delta_{\text {loc }}=\delta+\mathrm{d}$. The induced cohomology is the BRST-cohomology $\mathcal{H}_{B R S T}^{*}\left(\right.$ lie $\left.\mathcal{G}, \Omega_{\text {loc }}\right)$.

\subsection{4. "Proof" of the Theorem}

We sketch the proof of the above theorem:

1) For $q=0, p=1$ we have the following identifications:

$\mathcal{C}^{q, p}=\mathcal{C}^{0}\left(\mathfrak{l i} \mathcal{G}, \Omega^{1}(P, \mathbf{g})\right) \cong \Omega^{\mathbf{1}}(\mathbf{P}, \mathbf{g})$ Lie algebra valued 1 -forms and $\mathcal{A} \subset$ $\Omega^{1}(P, \mathbf{g})$. Then $\mathfrak{s}=\delta$ and for $A \in \mathcal{A}, X \in \mathfrak{l i e} \mathcal{G}$ we get:

$$
\mathfrak{s}(A)(X)=\delta A(X)=\sigma(X) \cdot A=\operatorname{ad}(X) \cdot A=D_{A} X=\mathrm{d} X+\frac{1}{2}[A, X]
$$

Also $\mathrm{d} \eta(X)=\mathrm{d}(\eta(X))=\mathrm{d} X$, and $[X, \eta](A)=[A, \eta(X)]=[A, X]$ hence

$$
\mathfrak{s} A(X)=(\mathrm{d} \eta)(X)+\frac{1}{2}[A, \eta](X)
$$

2) For $q=1, p=0$ we have the following identifications:

$\mathcal{C}^{q, p}=\mathcal{C}^{1}\left(\mathfrak{l i} \mathfrak{e} \mathcal{G}, \Omega^{0}(P, \mathbf{g})\right)=\operatorname{Hom}(\mathfrak{l i} \mathfrak{e} \mathcal{G}$, lie $\mathcal{G})$. For $\eta=\mathrm{id}:$ lie $\mathcal{G} \rightarrow$ lie $\mathcal{G}$; $\eta(X): P \rightarrow \mathbf{g}$. Then $\mathfrak{s}=-\frac{1}{2} \delta$ and for $X_{0}, X_{1} \in \mathfrak{l i e} \mathcal{G}$ we get:

$$
\begin{aligned}
\mathfrak{s} \eta\left(X_{0}, X_{1}\right) & =-\frac{1}{2}\left(\operatorname{ad}\left(X_{0}\right) \eta\left(X_{1}\right)-\operatorname{ad}\left(X_{1}\right) \eta\left(X_{0}\right)\right)-\eta\left[X_{0}, X_{1}\right] \\
& =-\frac{1}{2}\left(L_{X_{0}} X_{1}-L_{X_{1}} X_{0}-\left[X_{0}, X_{1}\right]\right) \\
& =-\frac{1}{2}\left[X_{0}, X_{1}\right]=-\frac{1}{2}\left[\eta\left(X_{0}\right), \eta\left(X_{1}\right)\right]=-\frac{1}{2}[\eta, \eta]\left(X_{0}, X_{1}\right) .
\end{aligned}
$$




\subsection{Anomalies}

The Noether current induced by the chiral symmetry (after quantization) for the free case $(\psi=0)$ i.e. for pure Yang-Mills becomes

$$
\begin{aligned}
\partial^{\mu} J_{\mu} & =-\frac{g^{2}}{8 \pi^{2}} \varepsilon^{\mu \nu \rho \tau} \operatorname{trace} F_{\mu \nu} F_{\rho \tau} \\
& =-\frac{1}{4} \pi^{2} \varepsilon^{\mu \nu \rho \tau} \operatorname{trace} \partial_{\mu}\left(A_{\nu} \partial_{\rho} A_{\tau}+\frac{2}{3} A_{\mu} A_{\rho} A_{\tau}\right) \\
& =\omega \neq 0 \text { anomaly, see }(72) .
\end{aligned}
$$

Note the similarity with the Chern-Simon Lagrangian

$$
\mathcal{L}(A)=\int_{M} \operatorname{trace}\left(A \mathrm{~d} A+\frac{2}{3} A^{3}\right) .
$$

We are going to derive a representation of the chiral anomaly $\omega$ in the BRST cohomology, i.e. $[\omega] \in \mathcal{H}_{B R S T}^{1,0}\left(\right.$ lie $\left.\mathcal{G}, \Omega_{\text {loc }}\right)$.

The question is, if $\mathfrak{s} \omega=0$, does there exist a local functional $F(A)$, such that $\omega=\mathfrak{s}(F(A))$ i.e., is $\omega$ BRST $\mathfrak{s}$-exact ? The answer in general is NO, i.e. $\omega$ represents a nontrivial cohomology class. This class is given by the Chern-Weil homotopy.

\subsubsection{The Chern-Weil Homotopy}

Let $\tilde{A}=A+\eta \in \mathcal{C}^{0,1} \times \mathcal{C}^{1,0}$ and $\tilde{F} \equiv \mathbf{s} \tilde{A}+\tilde{A}^{2}=F_{\tilde{A}}$. For $t \in[0,1]$ let $\tilde{F}_{t}=t \tilde{F}+\left(t^{2}-t\right) \tilde{A}^{2}$ and define the Chern-Simons form

$$
\omega_{2 q-1} \equiv q \int_{0}^{1} \operatorname{trace}\left(\tilde{A} \tilde{F}_{t}^{q-1}\right) \mathrm{d} t
$$

we get

$$
\mathfrak{s} \omega_{2 q-1}=\operatorname{trace} \tilde{F}^{q} .
$$

We write $\omega_{2 q-1}$ as sum of homogeneous terms in ghost number (upper index) and degree (lower index) $\omega_{2 q-1}=\omega_{2 q-1}^{0}+\omega_{2 q-2}^{1}+\omega_{2 q-3}^{2}+\cdots+\omega_{0}^{2 q-1}$. Let $\omega(X, A)=\int_{M} \omega_{2 q-2}^{1}(X)$.

Theorem 21. (Schmid [38]) The form $\omega(X, A)=\int_{M} \int_{0}^{1} \tilde{A} \tilde{F}_{t}^{q-1}(X) \mathrm{d} t$ satisfies the Wess-Zumino consistency condition $(\mathfrak{s} \omega)\left(X_{0}, X_{1}, A\right)=0$ and represents the chiral anomaly $[\omega] \in \mathcal{H}_{B R S T}^{1,0}\left(\mathfrak{l i e} \mathcal{G}, \Omega_{\mathrm{loc}}\right)$. 
We have an explicit form of the anomaly in $(2 q-2)$ dimensions:

$$
\omega_{2 q-2}^{1}=q(q-1) \int_{0}^{1}(1-t) \operatorname{trace}\left(\eta \delta_{\text {loc }}\left(\tilde{A} \tilde{F}_{t}^{q-2}\right)\right) \mathrm{d} t .
$$

So for $q=2$ the non-Abelian anomaly in 2-dim. becomes $\omega_{2}^{1}=\operatorname{trace}\left(\eta \delta_{\text {loc }} \tilde{A}\right)$, and for $q=3$ the non-Abelian anomaly in 4-dim. becomes

$$
\omega_{4}^{1}=\operatorname{trace}\left(\eta \delta_{\mathrm{loc}}\left(\tilde{A} \delta_{\mathrm{loc}} \tilde{A}+\frac{1}{2} \tilde{A}^{3}\right)\right) \text {. }
$$

\subsection{The Wess-Zumino Consistency Condition}

This is a problem in local cohomology. We consider the bicomplex $\mathcal{C}_{\text {loc }}^{*}=$ $\left\{\mathcal{C}^{q, p}, \Delta\right\}_{q, p \in \mathbb{N}}$ with total differential $\Delta=\delta_{\text {loc }}+(-1)^{p}$ d where $\delta_{\text {loc }}: \mathcal{C}^{q, p}\left(\right.$ lie $\left.\mathcal{G}, \Omega_{\text {loc }}\right)$ $\longrightarrow \mathcal{C}^{q+1, p}\left(\right.$ lie $\left.\mathcal{G}, \Omega_{\text {loc }}\right)$ and $\mathrm{d}: \mathcal{C}^{q, p}\left(\right.$ lie $\left.\mathcal{G}, \Omega_{\mathrm{loc}}\right) \longrightarrow \mathcal{C}^{q, p+1}\left(\right.$ lie $\left.\mathcal{G}, \Omega_{\mathrm{loc}}\right)$. We have $\Delta^{2}=\delta_{\text {loc }} \mathrm{d}+\mathrm{d} \delta_{\text {loc }}=\delta_{\text {loc }}^{2}=\mathrm{d}^{2}=0$.

The Wess-Zumino consistency condition for $\omega \in \mathcal{C}_{\text {loc }}^{*}$ means that there exists an $\alpha \in \mathcal{C}_{\text {loc }}^{*}$ such that

$$
\delta_{\mathrm{loc}} \omega+\mathrm{d} \alpha=0 .
$$

Any solution of (WZ) of the form $\omega=\delta_{\text {loc }} \beta+\mathrm{d} \gamma, \beta, \gamma \in \mathcal{C}_{\text {loc }}^{*}$ is trivial, i.e. $\delta_{\text {loc }} \omega=0$. The consistency condition (WZ) produces the so called descent equations. If $\delta_{\text {loc }} \omega+\mathrm{d} \alpha=0$ taking $\delta_{\text {loc }}$ of (WZ) we get $\delta_{\text {loc }}{ }^{2} \omega+\delta_{\text {loc }} \mathrm{d} \alpha=0$ hence $\delta_{\text {loc }} \mathrm{d} \alpha=0$. The Poincare lemma implies there exists a local form $\beta$ such that $\delta_{\text {loc }} \alpha+\mathrm{d} \beta=0$. By definition $\delta_{\text {loc }}[\omega]=[\alpha]$. If $\omega$ is trivial, i.e. $\omega=$ $\delta_{\text {loc }} \beta+\mathrm{d} \gamma$ then $\delta_{\text {loc }} \mathrm{d} \gamma=-\mathrm{d} \alpha$, hence $\alpha=\delta_{\text {loc }} \gamma+\mathrm{d} \lambda$, i.e. $[\alpha]=0$.

We get the descent equations

$$
\begin{aligned}
\delta_{\operatorname{loc} \omega+\mathrm{d} \omega_{1}} & =0 \\
\delta_{\operatorname{loc}} \omega_{1}+\mathrm{d} \omega_{2} & =0 \\
& \cdot \\
& \cdot \\
\delta_{\mathrm{loc}} \omega_{k-1}+\mathrm{d} \omega_{k} & =0
\end{aligned}
$$

where $k$ is the smallest integer such that $[\omega] \in \mathrm{H}_{\mathrm{loc}}^{k}(\mathfrak{l i e} \mathcal{G})$ with $\delta_{\mathrm{loc}} \omega=0$.

\subsection{1. g-Symplectic Structures}

Definition 22. A g-symplectic structure on $P$ is a g-form $\Omega \in \Omega^{2}(P, \mathbf{g})$ which is closed and nondegenerate, i.e. $\mathrm{d} \Omega=0$ and for each $p \in P$ the map $\Omega(p)$ : 
$T_{p} P \times T_{p} P \rightarrow \mathbf{g}$ is bilinear and nondegenerate. We have the induced linear injective map $\Omega(p)^{\#}: T_{p} P \rightarrow L\left(T_{p} P, \mathbf{g}\right) ; \Omega(p)^{\#}(v) \cdot w=\Omega(p)(v, w)$.

A vector field $X$ on $P$ is called $\mathbf{g}$-Hamiltonian if there exists a $\mathrm{g}$-function $f$ : $P \rightarrow \mathrm{g}$ such that $\mathrm{d} f=i_{X} \Omega$.

A g-vector field $X$ is locally $\mathbf{g}$-Hamiltonian iff its flow $\varphi_{t}$ is $\mathbf{g}$-symplectic, i.e. $\varphi_{t}^{*} \Omega=\Omega$. We have the

Lemma 23. Poincare lemma: For any $\alpha \in \Omega^{p}\left(\mathbb{R}^{n}, \mathbf{g}\right)$ with $\mathrm{d} \alpha=0$ there exists locally a $\beta \in \Omega^{p-1}\left(\mathbb{R}^{n}, \mathbf{g}\right)$ such that $\alpha=\mathrm{d} \beta$ and

$$
\beta(x)=\int_{0}^{1} i_{x} \alpha(t x) \mathrm{d} t .
$$

Theorem 24. (Schmid [39]) If $G$ is semi simple, then every $G$-orbit $\mathcal{O}_{p}$ of the right action of $G$ on $P$ is a $\mathrm{g}$-symplectic manifold induced by the Maurer Cartan form on $G$.

\subsubsection{The Canonical Momentum Map on $\mathcal{O}_{p}$}

Proposition 25. (Schmid [39]) For every $\xi \in \mathbf{g}$ the fundamental vector field $\xi_{P}$ on $\mathcal{O}_{p}$ defined by

$$
\xi_{P}(q)=\left.\frac{\mathrm{d}}{\mathrm{d} t}\right|_{t=0} R_{\exp t \xi}(q)
$$

is locally g-Hamiltonian.

Corollary 26. For every $\xi \in \mathbf{g}$ there exists a $\mathfrak{g}$-function $H: \mathcal{O}_{p} \rightarrow \mathbf{g}$ such that $\xi_{P}=X_{H}$, i.e. $\mathrm{d} H=i_{\xi_{P}} \Omega_{p}$. Explicitly

$$
H(x)=-\frac{1}{2}[x, x \cdot \xi]
$$

Proposition 27. (Schmid[39]) The g-momentum map $J: \mathcal{O}_{p} \rightarrow \mathcal{L}(\mathbf{g}, \mathbf{g})$ of the right action of $G$ on $\mathcal{O}_{p}$ defined by $\langle J(q), \xi\rangle=H(q), q \in \mathcal{O}_{p}, \xi \in \mathbf{g}$ is given by

$$
J(q)=\operatorname{ad}_{\eta} \circ T R_{q}
$$

where $\eta=R_{p}^{*} X_{t}(g), q=p \cdot g$. 


\subsubsection{A Solution of Consistency Condition}

The infinite dimensional analogue of the previous construction leads to the following: Consider a principal $\mathcal{G}$ bundle $(\mathcal{P}, \pi, \mathcal{M})$, where $\mathcal{P}=\Omega^{*}(P, \mathbf{g})$ with the adjoint $\mathcal{G}$ action and $\mathcal{M}=\mathcal{P} / \mathcal{G}$. For $A \in \Omega^{*}(P, \mathbf{g})$ the canonical 1 -form $\Theta_{A}$ on the orbit $\mathcal{O}_{A}$ induced from the Maurer-Cartan form on $\mathcal{G}$ becomes a map $\Theta_{A}: \mathcal{O}_{A} \rightarrow$ $\Omega^{1}(P, \mathbf{g}) \simeq \mathcal{C}_{\text {loc }}^{0,1}$ and the momentum map $J: \mathcal{O}_{A} \rightarrow \mathcal{L}($ lie $\mathcal{G}$, lie $\mathcal{G})=\mathcal{C}_{\text {loc }}^{1,0}$.

Theorem 28. (Schmid [39]) The momentum map J satisfies the consistency condition for the canonical 1-form (Maurer-Cartan) $\Theta_{A}$ of $\mathcal{G}$

$$
\delta_{\mathrm{loc}} \Theta_{A}+\mathrm{d} J=0 .
$$

\section{Lie groups of pseudodifferential and Fourier integral operators}

Pseudodifferential operators and Fourier integral operators are used in physics to construct solutions of partial differential equations, e.g. the fundamental solution of the heat equation is a pseudodifferential operator. They are also used to prove local formulas for index theorems. Geometric properties are used to describe Fourier integral operators as a symmetry group for the $\mathrm{KdV}$ equation as Hamiltonian system. These will be discussed in Section 8. These operators have very nice geometric properties which we will discuss here.

Fourier integral operators ( FIO for short) generalize pseudodifferential operators ( $\Psi D O$ for short) which themselves generalize differential operators ( $D O$ for short). So as sets we have the inclusions

$$
F I O \supset \Psi D O \supset D O
$$

\subsection{Pseudodifferential Operators $\Psi D O$}

Consider a differential operator $P$ on $\Omega \subset \mathbb{R}^{n}$ of order $m$ with smooth coefficients $a_{\alpha}$

$$
P u(x)=\sum_{|\alpha| \leq m} a_{\alpha}(x) D_{x}^{\alpha} u(x), \quad u \in C^{\infty}(\Omega) .
$$

We associate to the operator $P$ the polynomial

$$
p(x, \xi) \sum_{|\alpha| \leq m} a_{\alpha}(x) \xi^{\alpha}
$$


called the symbol of $P$. Using the Fourier transform $\hat{u}(\xi)$ of $u(x)$ we have $\widehat{D_{x}^{\alpha} u}(\xi)=\xi^{\alpha} \hat{u}(\xi)$ and $D_{x}^{\alpha} u(x)=(2 \pi)^{-n} \int \mathrm{e}^{\mathrm{i} x \cdot \xi} \xi^{\alpha} \hat{u}(\xi) \mathrm{d} \xi$ so we can write

$$
\begin{aligned}
P u(x) & =(2 \pi)^{-n} \int \mathrm{e}^{\mathrm{i} x \cdot \xi} p(x, \xi) \hat{u}(\xi) \mathrm{d} \xi \\
& =(2 \pi)^{-n} \iint \mathrm{e}^{\mathrm{i}(x-y) \cdot \xi} p(x, \xi) u(y) \mathrm{d} y \mathrm{~d} \xi .
\end{aligned}
$$

A pseudodifferential operator $P$ is of the form (89) but with symbol $p(x, \xi)$ of a more general class then polynomials. A smooth function $p(x, \xi)$ on $\Omega \times \mathbb{R}^{n}$ belongs to the symbol class $S_{\rho, \delta}^{m}(\Omega), 0 \leq \delta<\rho<1$ if for any compact $K \subset \Omega$, any $\alpha, \beta$ there exists a const. $C_{\alpha \beta}(K)>0$ such that for all $x \in K, \xi \in \mathbb{R}^{n}$

$$
\left|D_{x}^{\beta} D_{\xi}^{\alpha} p(x, \xi)\right| \leq C_{\alpha, \beta}(K)(1+|\xi|)^{m-\rho|\alpha|+\delta|\beta|} .
$$

We restrict ourselves to classical symbols, i.e. those who have an asymptotic expansion of the form

$$
p(x, \xi) \sim \sum_{j=0}^{\infty} p_{m-j}(x, \xi), m=\text { order of } p
$$

where each $p_{m-j}(x, \xi) \in C^{\infty}\left(\Omega \times \dot{\mathbb{R}}^{n}\right)$ is homogeneous of degree $m-j$ in $\xi$, i.e. $p_{m-j}(x, \tau \xi)=\tau^{m-j} p_{m-j}(x, \xi), \tau>0$. Then $P$ is a classical pseudodifferential operator of order $m$ if $P$ is of the form

$$
P u(x)=(2 \pi)^{-n} \iint \mathrm{e}^{\mathrm{i}(x-y) \cdot \xi} p(x, \xi) u(y) \mathrm{d} y \mathrm{~d} \xi
$$

with $p(x, \xi)$ a classical symbol of the form (91). The principal symbol of $P$ is the leading term $p_{m}(x, \xi)$.

Denote by $\Psi D O_{m}$ the space of classical pseudodifferential operators of order $m$ and let $\Psi D O=\bigcup_{m} \Psi D O_{m}$ be the space of all pseudodifferential operators of all order. $\Psi D O$ is an infinite dimensional graded Lie algebra with commutator bracket satisfying the following: if $P \in \Psi D O_{m}$ and $Q \in \Psi D O_{n}$ then $[P, Q]=$ $P Q-Q P \in \Psi D O_{m+n-1}$. Note that $\Psi D O_{1}$ the space of pseudodifferential operators of order 1 is an infinite dimensional Lie subalgebra of $\Psi D O$.

It is a natural question to ask whether there exist corresponding Lie groups having $\Psi D O_{1}$ and $\Psi D O$ as their Lie algebras respectively? The answer is YES! The Lie group that has $\Psi D O_{1}$ as its Lie algebra is the group of invertible Fourier integral operators of order zero, denoted by $\left(F I O_{0}\right)_{*}$ and the group for $\Psi D O$ is the group of all invertible Fourier integral operators, denoted by $F I O_{*}$. We shall now discuss these Lie group structures following Adams, Ratiu and Schmid [1-3]. 


\subsection{Fourier Integral Operators $F I O$}

Fourier integral operators generalize pseudodifferential operators by allowing more general phase functions $\varphi(x, y, \xi)$ then the one we have for pseudodifferential operators in $(92) \varphi(x, y, \xi)=\mathrm{i}(x-y) \cdot \xi$. These are given by generating functions of canonical transformations.

\subsubsection{Generating Functions for Canonical Transformations:}

Let $S: \Omega \times \mathbb{R}^{n} \rightarrow \mathbb{R}$ be a smooth function in a neighborhood of $\left(x_{0}, \xi_{0}\right) \in \Omega \times \mathbb{R}^{n}$ such that $\frac{\partial^{2} S(x, \xi)}{\partial x \partial \xi} \neq 0$. Then $\Phi(y, \xi)=(x, \eta)$ where $\eta=\frac{\partial S(x, \xi)}{\partial x}, y=$ $\frac{\partial S(x, \xi)}{\partial \xi}$ defines a local canonical transformation

$$
\Phi:\left(y_{0}, \xi_{0}\right) \in \dot{T}^{*} \mathbb{R}^{n} \rightarrow\left(x_{0}, \eta_{0}\right) \in \dot{T}^{*} \Omega, \Phi^{*} \omega=\omega
$$

where $\omega=\sum_{i=1}^{n} \mathrm{~d} p_{i} \wedge \mathrm{d} q_{i}$ is the canonical symplectic form on $T^{*} \mathbb{R}^{n}$. The function $S$ is called the generating function of $\Phi$. Vice versa, every canonical transformation $\Phi$ has a locally generating function $S$. Note that $S$ is homogeneous of degree one in $\xi$ if and only if $\Phi$ is homogeneous in $\xi$.

Example: Let $S(x, \xi)=x \cdot \xi$. Then $\eta=\frac{\partial S}{\partial x}=\xi, y=\frac{\partial S}{\partial \xi}=x$ hence $\Phi=\mathrm{id}$.

\subsubsection{Fourier Integral Operators}

Let $S(x, \xi)$ be a generating function and $a(x, \xi)$ a classical symbol order $m$. Define a classical Fourier integral operator $A$ of order $m$ by

$$
\begin{aligned}
A u(x) & :=\int \mathrm{e}^{\mathrm{i} S(x, \xi)} a(x, \xi) \hat{u}(\xi) \mathrm{d} \xi \\
& =(2 \pi)^{-n} \iint e^{\mathrm{i}(S(x, \xi)-y \cdot \xi)} a(x, \xi) u(y) \mathrm{d} y \mathrm{~d} \xi
\end{aligned}
$$

More generally, a Fourier integral operator $A$ of order $m$ is defined by

$$
A u(x)=(2 \pi)^{-n} \iint \mathrm{e}^{\mathrm{i} \varphi(x, y, \xi)} a(x, \xi) u(y) \mathrm{d} y \mathrm{~d} \xi
$$

where $\varphi(x, y, \xi)$ is nondegenerate phase function (homogeneous +1 ) and the symbol $a(x, \xi) \in S_{\rho, \delta}^{m}$. 
Notice if $S(x, \xi)=x \cdot \xi$ or general $\varphi(x, y, \xi)=(x-y) \cdot \xi$ then the operator $A$ is a pseudodifferential operator defined by (92) and we have FIO $\supset$ FO $\supset D O$.

These Fourier integral operators are singular operators but make sense as oscillatory integrals and they have nice properties in the following sense:

Property 1) Fourier integral operators are invariant under diffeomorphisms. So they can be defined on manifolds $M$ as bounded linear operators $A: C^{\infty}(M) \rightarrow$ $C^{\infty}(M),(M$ compact!) such that $A$ is locally of the form (95); moreover, they extend continuously to distributions $A: \mathcal{E}^{\prime}(M) \rightarrow \mathcal{D}^{\prime}(M)$. Any $P \in \Psi D O_{m}$ extends as bounded linear operator $P: H_{c}^{s}(M) \rightarrow H_{c}^{s-m}(M)$.

Property 2) Pseudodifferential operators have properties which are close to differential operators (DO):

$P \in D O \Leftrightarrow P$ is local, i.e. supp $P u \subset$ supp $u$.

$P \in \Psi D O \Rightarrow P$ is pseudolocal, i.e. sing supp $P u \subset$ sing supp $u$.

Property 3) Pseudodifferential operators preserves the wave front sets $W F$ i.e. $W F(P u) \subset W F(u)$ where $W F(u) \subset \dot{T}^{*} M, \tau_{M}^{*} W F(u)=\operatorname{sing}$ supp $u$.

Fourier integral operators generalize pseudodifferential operators in the sense that they move the wave front sets $W F$ by a canonical relation $\Lambda$, i.e. if $A \in F I O$ then $W F(A u) \subset \Lambda \circ W F(u)$ where the canonical relation $\Lambda \subset \dot{T}^{*} M \times \dot{T}^{*} M$ is a conic Lagrangian submanifold, locally generated by the phase function $\varphi(x, y, \xi)$, i.e.

$$
\Lambda=\left\{\left(x, y, \mathrm{~d}_{(x, y)} \varphi(x, y, \xi)\right) ; \mathrm{d}_{\xi} \varphi=0\right\}
$$

\section{Remarks:}

a) If $\Lambda=\Delta=((x, \xi),(x, \xi))$ the diagonal then $A \in \Psi D O$.

b) Any $A \in F I O$ is determined by its symbol $a(x, \xi)$ and canonical relation $\Lambda$.

c) The principal symbol is globally defined $a_{m}(x, \xi): \dot{T}^{*} M \rightarrow \mathbb{R}$.

Property 4) Fourier integral operators are closed under multiplication: Assume $\Phi: \dot{T}^{*} M \rightarrow \dot{T}^{*} M$ is a globally defined canonical transformation, i.e. $\Phi^{*} \omega=\omega$, which is locally generated by $S(x, \xi)$. Denote by $F I O_{m}(\Phi)$ the space of Fourier integral operators of order $m$ associated to the canonical relation $\Lambda=\operatorname{graph}(\Phi)$. If $A_{1} \in F I O_{m_{1}}\left(\Phi_{1}\right)$ and $A_{2} \in F I O_{m_{2}}\left(\Phi_{2}\right)$ then $A_{1} \circ A_{2} \in F I O_{m_{1}+m_{2}}\left(\Phi_{1} \circ\right.$ $\left.\Phi_{2}\right)$. If $A \in F I O_{m}(\Phi)$ and $A^{-1} \in F I O$ exists, then $A^{-1} \in F I O_{m}\left(\Phi^{-1}\right)$. Note that if $\Phi=$ id $: \dot{T}^{*} M \rightarrow \dot{T}^{*} M$ then $F I O_{m}($ id $)=\Psi D O_{m}$, i.e. Fourier integral operators associated with the identity are pseudodifferential operators.

Example: Let $f: M \rightarrow M$ be a diffeomorphism. Then

$$
f^{*} u(x)=(2 \pi)^{-n} \iint \mathrm{e}^{\mathrm{i}(f(x)-y) \cdot \xi} u(y) \mathrm{d} y \mathrm{~d} \xi
$$


defines a $F I O A=f^{*}: C^{\infty}(M) \rightarrow C^{\infty}(M)$ whose phase function generates the canonical cotangent lift $T^{*} f: \dot{T}^{*} M \rightarrow \dot{T}^{*} M$.

Notation: Denote by $F I O_{*}, \Psi D O_{*},\left(F I O_{m}\right)_{*},\left(\Psi D O_{m}\right)_{*}$ the invertible elements in the corresponding spaces, and $\operatorname{Diff}_{\theta}^{\infty}\left(\dot{T}^{*}(M)\right.$ the group of $\theta$ preserving diffeomorphisms on $\dot{T}^{*}(M)$ where $\theta=\sum p_{i} \mathrm{~d} q_{i}$ is the canonical 1-form. Note that $F I O_{*}, \Psi D O_{*},\left(F I O_{0}\right)_{*},\left(\Psi D O_{0}\right)_{*}$ are groups under operator multiplication.

\subsubsection{Exact Sequence}

Let $S(x, \xi)$ be the generating function of $\Phi$. Since $S$ is homogeneous of degree +1 in $\xi$, this implies that $\Phi$ homogeneous of degree +1 in $\xi$. Together with $\Phi^{*} \omega=\omega$ we get $\Phi^{*} \theta=\theta$ hence $\Phi \in \operatorname{Diff}_{\theta}^{s}\left(\dot{T}^{*} M\right)$. So we get a surjective map

$$
p: F I O_{*} \rightarrow \operatorname{Diff}_{\theta}^{s}\left(\dot{T}^{*} M\right), p(A)=\Phi
$$

where $\operatorname{graph}(\Phi)=\Lambda$ the canonical relation of $A$. The kernel of $p$ is $p^{-1}(e)=$ $\Psi D O_{*}, e=\mathrm{id}_{T^{*} M}$. Both $\Psi D O_{*}$ and $F I O_{*}$ are groups under operator multiplication, graded by the order (which is additive) and $p$ is a group homomorphism $p(A \circ B)=p(A) \circ p(B)$. So we get an exact sequence of groups ( $j$ the inclusion)

$$
I \longrightarrow \Psi D O_{*} \stackrel{j}{\hookrightarrow} F I O_{*} \stackrel{p}{\longrightarrow} \operatorname{Diff}_{\theta}^{s}\left(\dot{T}^{*} M\right) \longrightarrow e .
$$

We want to make this into an exact sequence of LIE GROUPS.

Notice that the zero order operators $\left(\Psi D O_{0}\right)_{*}$ and $\left(F I O_{0}\right)_{*}$ are groups and form exact sequence

$$
I \longrightarrow\left(\Psi D O_{0}\right)_{*} \stackrel{j}{\hookrightarrow}\left(F I O_{0}\right)_{*} \stackrel{p}{\longrightarrow} \operatorname{Diff}_{\theta}^{s}\left(\dot{T}^{*} M\right) \longrightarrow e .
$$

First we are going to give ILH-Lie group structures to this sequence (98) of zero order operators, then we'll move these structures by a fixed elliptic operator $T$ to any order $m$, e.g. $T=(1+\Delta)^{m / 2}: F I O_{0} \rightarrow F I O_{m}$.

For the parameter spaces we look at the corresponding Lie algebras of

$$
I \longrightarrow\left(\Psi D O_{0}\right)_{*} \stackrel{j}{\hookrightarrow}\left(F I O_{0}\right)_{*} \stackrel{p}{\longrightarrow} \operatorname{Diff}_{\theta}^{s}\left(\dot{T}^{*} M\right) \longrightarrow e .
$$

The Lie algebras are

$$
0 \longrightarrow \Psi D O_{0} \stackrel{j}{\longrightarrow} \Psi D O_{1} \stackrel{\pi}{\longrightarrow} C_{+1}^{\infty}\left(\dot{T}^{*} M\right) \longrightarrow 0
$$


where $\pi(P)$ is the principal symbol (homog. +1 ) of $P \in \Psi D O_{1}$. The space of homogeneous functions $C_{+1}^{\infty}\left(\dot{T}^{*} M\right)$ is isomorphic to the space of globally Hamiltonian vector fields $C_{+1}^{\infty}\left(\dot{T}^{*} M\right) \cong \mathfrak{X}_{\theta}^{\infty}\left(\dot{T}^{*} M\right)=\left\{X ; L_{X} \theta=0\right\}$, as discussed in Section 4.4.

Remark: Clearly FIO $\neq \exp (\Psi D O)$ since $\operatorname{Diff}_{\theta}^{s}\left(\dot{T}^{*} M\right) \neq \exp \left(\mathfrak{X}_{\theta}^{s}\left(\dot{T}^{*} M\right)\right.$, i.e. we cannot obtain a chart at the identity in $F I O_{*}$ by exponentiating the Lie algebra $\Psi D O$.

The idea to construct a manifold and a Lie group structure on $F I O_{*}$ is to construct an infinite dimensional principal fiber bundle with

- base space $=\operatorname{Diff}_{\theta}^{s}\left(\dot{T}^{*} M\right)$

- total space $=\left(F I O_{0}\right)_{*}$

- fiber $=p^{-1}(\Phi)=\left(F I O_{0}(\Phi)\right)_{*} \cong\left(\Psi D O_{0}\right)_{*}$

- structure group $=\left(\Psi D O_{0}\right)_{*}$.

We outline this construction in 7 steps (for details see Adams, Ratiu and Schmid [1-3]:

Step 1: We show that $\operatorname{Diff}_{\theta}^{\infty}=\lim \operatorname{Diff}_{\theta}^{s}\left(\dot{T}^{*} M\right)$ is an ILH-Lie group.

Step 2: We show that $\left(\Psi D O_{0}\right)_{*}=\lim _{\leftarrow}\left(\Psi D O_{0}^{s}\right)_{*}$ is an ILH-Lie group.

Step 3: We piece $1 \& 2$ together via a local section $\sigma: U \subset \operatorname{Diff}_{\theta}^{s}\left(\dot{T}^{*} M\right) \rightarrow$ $\left(F I O_{0}\right)_{*}$. Then $\left(F I O_{0}\right)_{*}$ is locally of the form $\sigma(U) \simeq U \times\left(\Psi D O_{0}\right)_{*}$. This gives a chart at the identity $I \in\left(F I O_{0}\right)_{*}$.

Step 4: We move this chart around by the group the structure of $\operatorname{Diff}_{\theta}^{s}\left(\dot{T}^{*} M\right)$ $\Rightarrow\left(F I O_{0}\right)_{*}$ is a topological group.

Step 5: We check that the chart transitions are smooth $\Rightarrow\left(F I O_{0}\right)_{*}$ is a smooth manifold.

Step 6: We check that multiplication is "smooth" $\Rightarrow\left(F I O_{0}\right)_{*}$ is an ILH-Lie group.

Step 7: We identify $(1-\Delta)^{m / 2}:\left(F I O_{0}\right)_{*} \stackrel{\sim}{\rightarrow}\left(F I O_{m}\right)_{*} \Rightarrow F I O_{*}$ is an ILH-Lie group.

Let us go through some more details.

\subsubsection{Step 1: $\operatorname{Diff}_{\theta}^{s}\left(\dot{T}^{*} M\right)$ as ILH Lie Group}

In Section 4.3 we proved the following Theorem 16:

Theorem 29. $\operatorname{Diff}_{\theta}^{\infty}=\lim _{\infty \leftarrow s} \operatorname{Diff}_{\theta}^{s}\left(\dot{T}^{*} M\right)$ is an ILH Lie group where $\operatorname{Diff}_{\theta}^{s}\left(\dot{T}^{*} M\right)$ is isomorphic to the semidirect product

$$
\operatorname{Diff}_{\theta}^{s}\left(S T^{*} M\right)=\left\{(\varphi, h) \in \operatorname{Diff}^{s}\left(S T^{*} M\right) \ltimes C^{s}\left(S T^{*} M\right) ; \varphi^{*} \theta_{S}=h \theta_{S}\right\}
$$


with ILH Lie algebra $\mathfrak{X}_{\theta}^{s}\left(S\left(T^{*} M\right)\right)=\left\{Y \in \mathfrak{X}^{s}\left(S\left(T^{*} M\right)\right) ; L_{Y} \theta=0\right\}$ isomorphic to $C_{+1}^{s}\left(\dot{T}^{*} M\right)=\left\{H \in C^{s}\left(T^{*} M, \mathbb{R}\right) ; H\right.$ homogeneous of degree one $\}$.

\subsubsection{Step 2: $\left(\Psi D O_{0}\right)_{*}$ as ILH Lie Group}

We define an $H^{s}$ norm on $\Psi D O_{0}$ and complete this space to $\Psi D O_{0}^{s}$ which is a Hilbert algebra, hence $\left(\Psi D O_{0}^{s}\right)_{*}$ is a Hilbert Lie group. The topology is determined by the symbols of pseudodifferential operators. If we want to define seminorms on $\Psi D O_{0}$ directly we would end up with a Frechet space because each $P \in \Psi D O_{0}$ has a symbol of the form $p(x, \xi)=\sum_{j=0}^{-\infty} p_{j}(x, \xi)$, so we would have to control an infinite number of functions and their derivatives, and an infinite product of Hilbert spaces is no longer a Hilbert space. So what we do is we cut the symbol at the term $p_{-k}$ for some fixed $k<\infty$. In terms of the operators, we look at the quotient spaces $\Psi D O_{m, k}:=\Psi D O_{m} / \Psi D O_{-k-1}$ and similarly $F I O_{m, k}(\Phi):=F I O_{m}(\Phi) / F I O_{-k-1}(\Phi), F I O_{m, k}=\cup_{\Phi} F I O_{m, k}(\Phi)$, where $F I O_{m}(\Phi)=\left\{A \in F I O_{m} ; p(A)=\Phi\right\}$.

Composition is still well defined in $\Psi D O_{0, k}$ and $F I O_{0, k}$ and we denote by $\left(\Psi D O_{0, k}\right)_{*}$ and $\left(F I O_{0, k}\right)_{*}$ the groups of invertible elements in $\Psi D O_{0, k}$ and $F I O_{0, k}$ respectively. We still have the exact sequence of groups:

$$
I \rightarrow\left(\Psi D O_{0, k}\right)_{*} \stackrel{j}{\hookrightarrow}\left(F I O_{0, k}\right)_{*} \stackrel{p}{\rightarrow} \operatorname{Diff}_{\theta}^{s}\left(\dot{T}^{*} M\right) \rightarrow \mathrm{id}
$$

For $P \in \Psi D O_{m, k}$ with symbol $p(x, \xi)=p_{m}(x, \xi)+\cdots+p_{-k}(x, \xi)$ we define the norm by

$$
\|P\|_{m+k, s}^{2}=\left\|\tilde{p}_{m}\right\|_{s+k+m}^{2}+\left\|\tilde{p}_{m-1}\right\|_{s+k+m-1}^{2}+\cdots+\left\|\tilde{p}_{k}\right\|_{s}^{2}
$$

where $\tilde{p}_{m-j}$ is the restriction of $p_{m-j}$ to the cosphere bundle $S\left(T^{*} M\right)$ and $\left\|\tilde{p}_{m-j}\right\|_{s+k+m-j}^{2}$ is the $H^{s+k+m-j}-$ Sobolev norm on $S\left(T^{*} M\right)$. Let $\Psi D O_{m, k}^{s}$ denote the completion of $\Psi D O_{m, k}$ with respect to this norm and for $m=0$ denote by $\left(\Psi D O_{0, k}^{s}\right)_{*}$ the group of invertible elements in $\Psi D O_{0, k}^{s}$.

Theorem 30. (Adams-Ratiu-Schmid [2]) For each $s>n$ the group $\left(\Psi D O_{0, k}^{s}\right)_{*}$ is a Hilbert Lie group with Lie algebra $\Psi D O_{0, k}^{s}$. That means $\left(\Psi D O_{0, k}^{s}\right)_{*}$ is a smooth $\left(C^{\infty}\right)$ Hilbert manifold with smooth group operations. Moreover the inverse limit $\left(\Psi D O_{0, k}\right)_{*}=\lim _{\infty \leftarrow s}\left(\Psi D O_{0, k}^{s}\right)_{*}$ is an ILH Lie group.

At the end of the day we will take the limit $k \rightarrow \infty$ ! 


\subsubsection{Step 3: The Local Section:}

We piece together $\operatorname{Diff}_{\theta}^{s}\left(\dot{T}^{*} M\right)$ and $\Psi D O_{0}^{s}$ by a local section

$$
\sigma: \mathcal{U} \subset \operatorname{Diff}_{\theta}^{s}\left(\dot{T}^{*} M\right) \rightarrow\left(F I O_{0}\right)_{*}
$$

where $\mathcal{U}$ is a neighborhood of id $\in \operatorname{Diff}_{\theta}^{s}\left(\dot{T}^{*} M\right)$. This gives $\left(F I O_{0}\right)_{*}$ a local product structure

$$
\sigma(\mathcal{U}) \cong \mathcal{U} \times\left(\Psi D O_{0}\right)_{*}
$$

defined by $A \mapsto\left(p(A), A \circ \sigma(p(A))^{-1}\right)$, and inverse $(\varphi, P) \mapsto P \cdot \sigma(\varphi)$. Hence we get a chart at the identity $I \in\left(F I O_{0}\right)_{*}$.

Problem: Fourier integral operators are only locally defined but we need a global writing of Fourier integral operators, i.e. a global phase function for Fourier integral operators close to $I$. This is done by constructing an explicit chart about id of $\operatorname{Diff}_{\theta}^{s}\left(\dot{T}^{*} M\right)$ in the following manner.

Theorem 31. (Adams-Ratiu-Schmid [2])

A) Let $H \in C_{+1}^{s+1}\left(\dot{T}^{*} M\right)$ close to zero and define $\varphi_{H}: \dot{T}^{*} M \times M \rightarrow \mathbb{R}$

$$
\varphi_{H}\left(\alpha_{x}, y\right):=\alpha_{x} \cdot\left(\exp _{x}^{-1}(y)\right)+H\left(\alpha_{x}\right)
$$

where exp is defined by a Riemannian metric on $M$. Then there exists an $\Phi \in$ $\operatorname{Diff}_{\theta}^{s}\left(\dot{T}^{*} M\right)$ close to id such that $\varphi_{H}$ is a global phase function for graph $(\Phi)$.

B) The map $H \leftrightarrow \Phi$ is a bijection from a neighborhood $\mathcal{V}(0) \subset C_{+1}^{s+1}\left(\dot{T}^{*} M\right)$ onto a neighborhood $\mathcal{U}(e) \subset \operatorname{Diffs}_{\theta}^{s}\left(\dot{T}^{*} M\right)$.

For the inverse let $\Phi \in \operatorname{Diff}_{\theta}^{s}\left(\dot{T}^{*} M\right)$ be close to $e\left(\tau^{*}: T^{*} M \rightarrow M\right.$ is the projection) and define $H: \dot{T}^{*} M \rightarrow \mathbb{R}$ by

$$
H\left(\alpha_{x}\right)=-\alpha_{x} \cdot \exp _{x}^{-1}\left(\tau^{*} \Phi^{-1}\left(\alpha_{x}\right)\right) .
$$

Then $\varphi_{H}$ defined by (104) is a global phase function for graph $(\Phi)$.

Now we define a local section $\sigma$ of the sequence (98)

$$
\sigma: \mathcal{U} \subset \operatorname{Diff}_{\theta}^{s}\left(\dot{T}^{*} M\right) \rightarrow\left(F I O_{0}\right)_{*}
$$

as follows: let $\Phi \in \mathcal{U} \subset \operatorname{Diff}_{\theta}^{s}\left(\dot{T}^{*} M\right)$ (close to id ) and define $\sigma(\Phi)$ by

$$
\sigma(\Phi) u(x):=(2 \pi)^{-n} \int_{T_{x}^{*} M} \int_{B_{\delta}(x)} \chi(x, y) \mathrm{e}^{\mathrm{i} \varphi_{H}\left(\alpha_{x}, y\right)} u(y)\left|\operatorname{det} \exp _{x}\right| \mathrm{d} y \mathrm{~d} \xi
$$


where $B_{\delta}(x)$ is the open neighborhood of $x$, where $\exp _{x}$ is a local diffeomorphism, and $\chi(x, y)$ is a bump function.

Then $\sigma(\Phi)$ is a Fourier integral operator with smooth phase function $\varphi_{H}$, the global generating function of $\operatorname{graph}(\Phi)$ and amplitude $a=1$. Notice that $H$ is smooth if $\Phi$ is smooth, in which case $\sigma(\Phi)$ is a well defined Fourier integral operator of order zero. Moreover, $\sigma(\Phi)$ is invertible modulo smoothing operators since $\Phi$ is invertible and its principal symbol is $a=1$, hence $\sigma(\Phi) \in\left(F I O_{0}\right)_{*}$. In particular $\sigma(\Phi) \in\left(F I O_{0, k}\right)_{*}$ for any $k$. Furthermore, $p(\sigma(\Phi))=\Phi$ for any $\Phi \in \mathcal{U}$, hence $\sigma$ is a local section of the exact sequences (98) and (101). We use this local section $\sigma$ to give $\left(F I O_{0}\right)_{*}$ the local product structure

$$
p^{-1}(\mathcal{U}) \simeq \mathcal{U} \times\left(\Psi D O_{0}\right)_{*}
$$

Now we define the topology around the identity in $\left(F I O_{0, k}\right)_{*}$ by the bijection $\beta: p^{-1}\left(U^{2 t}\right) \rightarrow U^{2 t} \times\left(\Psi D O_{0, k}^{2(t-k)}\right)_{*}: \beta(A)=\left(p(A), A \circ \sigma(p(A))^{-1}\right)$ and $\beta^{-1}(\Phi, P)=P \circ \sigma(\Phi)$, where $U^{2 t}=\mathcal{U} \cap \operatorname{Diff}_{\theta}^{2 t}$. This defines a local chart at the identity $I \in\left(F I O_{0, k}\right)_{*}$.

\subsubsection{Step 4: $\left(\mathrm{FIO}_{0}\right)_{*}$ as Topological Group}

We move this chart (constructed in step 3) around by the group structure of $\operatorname{Diff}_{\theta}^{s}\left(\dot{T}^{*} M\right)$. Compatibility conditions for the group structure and the topology give conditions on $\sigma$ to make $\left(F I O_{0}\right)_{*}$ a topological group.

To define the topology on $\left(F I O_{0, k}\right)_{*}$ we move the open set $p^{-1}\left(U^{2 t}\right)$ by right translations (smooth!). We complete this topological space in the right-uniform structure and denote it by $\left(F I O_{0, k}^{t}\right)_{*}$. For each $t>n / 2$ we obtain $\left(F I O_{0, k}^{t}\right)_{*}$ as a topological group and $\left(F I O_{0, k}\right)_{*}=\bigcap_{t}\left(F I O_{0, k}^{t}\right)_{*}$ with the inverse limit topology is a topological group as well.

To prove this, we have to show that the map $(A, B) \mapsto A B^{-1}$ is continuous for any $A, B \in\left(F I O_{0, k}^{t}\right)_{*}$. This amounts to show that the following map in local coordinates is continuous:

$$
\begin{array}{r}
\left(U^{2 t} \times \Psi D O_{0, k}^{2(t-k)}\right) \times\left(U^{2 t} \times \Psi D O_{0, k}^{2(t-k)}\right) \rightarrow\left(U^{2 t} \times \Psi D O_{0, k}^{2(t-k)}\right) \\
\left(\left(\Phi_{1}, P_{1}\right),\left(\Phi_{2}, P_{2}\right)\right) \mapsto\left(\Phi_{1} \circ \Phi_{2}^{-1}, P_{1} \sigma\left(\Phi_{1}\right) \sigma\left(\Phi_{2}\right)^{-1} P_{2}^{-1} \sigma\left(\Phi_{1} \circ \Phi_{2}^{-1}\right)^{-1}\right)
\end{array}
$$

which involves a very careful study of products of symbols of FIOs. 


\subsubsection{Step 5: $\left(\mathrm{FIO}_{0}\right)_{*}$ as Smooth Manifold}

Overlap conditions in local charts give conditions on $\sigma$ to make $\left(F I Q_{0}\right)_{*}$ into a smooth manifold. To prove that the transition maps between local charts are smooth it amounts to show that the following map is differentiable

$$
\begin{gathered}
\left(U^{2 t} \cdot \alpha \cap U^{2 t} \cdot \beta\right) \times\left(\Psi D O_{0, k}^{2(t-k)}\right)_{*} \rightarrow\left(\Psi D O_{0, k}^{2(t-k)}\right)_{*} \\
(\Phi, P) \mapsto P \sigma\left(\Phi \circ \alpha^{-1}\right) A B^{-1} \sigma\left(\Phi \circ \beta^{-1}\right)^{-1}
\end{gathered}
$$

for any $A, B \in\left(F I O_{0, k}^{t}\right)_{*}$, where $\alpha=p(A), \beta=p(B)$. The symbol calculus shows that this map is of class $C^{t}$, hence $\left(F I O_{0, k}^{t}\right)_{*}$ is a smooth manifold of class $C^{t}$.

\subsubsection{Step 6: $\left(\mathrm{FIO}_{0}\right)_{*}$ as ILH Lie Group}

We check smoothness of multiplication and inversion

$$
\begin{aligned}
& \mu:\left(F I O_{0}\right)_{*} \times\left(F I O_{0}\right)_{*} \rightarrow\left(F I O_{0}\right)_{*}, \quad \mu(A, B)=A \circ B \\
& \nu:\left(F I O_{0}\right)_{*} \rightarrow\left(F I O_{0}\right)_{*}, \quad \nu(A)=A^{-1} .
\end{aligned}
$$

To show that the group multiplication in $\left(F I O_{0, k}^{t}\right)_{*}$ is smooth it amounts to show that the following map is differentiable

$$
\begin{aligned}
& \left(U^{2(t+s)} \cdot \alpha\right) \times\left(\Psi_{0, k}^{2(t+s+k)}\right)_{*} \times\left(U^{2(t+s)} \cdot \beta\right) \times\left(\Psi_{0, k}^{2(t+s+k)}\right)_{*} \\
& \rightarrow\left(U^{2(t+s)} \cdot(r \alpha \cdot \beta) \times\left(\Psi_{0, k}^{2(t+s+k)}\right)_{*}\right. \\
& \left(\left(\Phi_{1}, P_{1}\right),\left(\Phi_{2}, P_{2}\right)\right) \mapsto \\
& \quad\left(\Phi_{1} \circ \Phi_{2}, P_{1} \sigma\left(\Phi_{1} \circ \alpha^{-1}\right) A P_{2} \sigma\left(\Phi_{2} \beta^{-1}\right) A^{-1} \sigma\left(\Phi_{1} \Phi_{2} \beta^{-1} \alpha^{-1}\right)^{-1}\right)
\end{aligned}
$$

for any $A \in\left(F I O_{0, k}^{t+s}\right)_{*}, B \in\left(F I O_{0, k}^{t}\right)_{*}$ where $\alpha=p(A), \beta=p(B)$.

This makes $\left(F I O_{0}\right)_{*}=\lim _{\infty \leftarrow s}\left(F I O_{0}^{s}\right)_{*}$ into an ILH Lie group.

\subsubsection{Step 7: $F I O_{*}$ as Lie Group}

To obtain a Lie group structure on $F I O_{*}$ of all invertible Fourier integral operators we use the Laplace operator to identify $(1-\Delta)^{m / 2}:\left(F I O_{0}\right)_{*} \stackrel{\sim}{\rightarrow}\left(F I O_{m}\right)_{*}$ and induce the corresponding manifold structure on $F I O_{*}$. Multiplication has 
the same smoothness properties as before between the appropriate spaces, which makes $F I O_{*}$ into an ILH Lie group.

The final result is the following:

Theorem 32. (Main Theorem) (Adams, Ratiu and Schmid [1-3]) The group $\mathrm{FIO}_{*}(M)$ of invertible Fourier integral operators on a compact manifold $M$ is a graded infinite dimensional ILH-Lie group with graded infinite dimensional Lie algebra $\Psi D O(M)$ of all pseudodifferential operators on $M . F I O_{*}(M)$ is and infinite dimensional principal fiber bundle over the base manifold $\operatorname{Diff}_{\theta}^{s}\left(\dot{T}^{*} M\right)$ of contact transformations of $\dot{T}^{*} M$ with gauge group $\Psi D O_{*}(M)$ of invertible pseudodifferential operators.

We have the following smoothness properties:

$$
\left(F I O_{0, k}\right)_{*}=\lim _{\infty \leftarrow t}\left(F I O_{0, k}^{t}\right)_{*} \text { is an ILH Lie group }
$$

the multiplication $\mu:\left(F I O_{0, k}^{t+p}\right)_{*} \times\left(F I O_{0, k}^{t}\right)_{*} \rightarrow\left(F I O_{0, k}^{t}\right)_{*}: \mu(A, B)=A B$ is $C^{p}$ differentiable,

the inversion $\nu:\left(F I O_{0, k}^{t+p}\right)_{*} \rightarrow\left(F I O_{0, k}^{t}\right)_{*}: \nu(A)=A^{-1}$ is $C^{p}$ differentiable, right multiplications $R_{A}:\left(F I O_{0, k}^{t}\right)_{*} \rightarrow\left(F I O_{0, k}^{t}\right)_{*}: R_{A}(B)=B A$ are $C^{t}$ differentiable, for any $A \in F I O_{0, k}^{t}$

the left multiplications $L_{A}:\left(F I O_{0, k}^{t}\right)_{*} \rightarrow\left(F I O_{0, k}^{t}\right)_{*}: L_{A}(B)=A B$ are $C^{0}$ (continuous), for any $A \in F I O_{0, k}^{t}$.

\section{7. $\operatorname{Diff}(M)$ and $F I O$ for non-compact manifolds. Application to fluid dynamics and quantization}

The "classical" theory of diffeomorphism groups and the groups of Fourier integral operators was developed in the 1970's and 1980's. This was all done for compact manifolds, mainly for technical reasons. Many attempts to deal with noncompact manifolds failed, until in the 1990's after J. Eichhorn published his papers on the manifold structures of maps between open manifolds [14] and we joined forces to overcome the technical difficulties one encounters dealing with noncompact manifolds. Two typical quotations about the non-compact case:

1) "Topology \& Analysis: Atiyah-Singer Index Formula \& Gauge Theoretic Physics" (Boos-Bleecker [7], p. 182):

In what follows, the manifolds $M$ is "closed" i.e. compact, without boundary. 
We make this convention in part for convenience (in order to make some proofs go easier) but also because otherwise some of the following theorems would be meaningless or false.

2) J. Eichhorn: "There is exactly one thing that work in the non-compact case: NOTHING”

Example of what's going wrong: Let $M, N$ be compact manifolds, then $f$ : $M \rightarrow N$ is of Sobolev class $H^{s} \Longleftrightarrow$ the local representatives $f_{j}^{i}: U_{i} \subset \mathbb{R}^{m} \rightarrow$ $V_{j} \subset \mathbb{R}^{n}$ are of class $H^{s}$, where $M=\bigcup\left(U_{i}, \phi_{i}\right), N=\bigcup\left(V_{j}, \psi_{j}\right), f_{j}^{i}:=$ $\psi_{j} \circ f \circ \phi_{i}^{-1}$. These covers are finite if $M, N$ are compact. This definition is invariant $\Leftrightarrow s>\frac{n}{2}+1$. In the compact case we can define the distance by

$$
d^{s}(f, g):=\left(\sum_{i, j}\left\|f_{j}^{i}-g_{j}^{i}\right\|_{s}^{2}\right)^{\frac{1}{2}}
$$

These definitions are meaningless if $M$ and $N$ are open!

\subsection{Bounded Geometry}

The idea of overcoming compactness is to use bounded geometry, which means that we will have control over the metric and its derivatives and the maps are adapted to the bounded geometry, i.e. we have control over the mappings and their derivatives by the metric as well.

Definition 33. A Riemannian manifold $\left(M^{n}, g\right)$ has bounded geometry of order $k, 0 \leq k \leq \infty$, if it has a positive injectivity radius and the curvature and all is derivatives up to order $k$ are uniformly bounded; i.e the following two conditions are satisfied:

$$
\begin{gathered}
\mathrm{I}): r_{i n j}(M)=\inf _{x \in M} r_{i n j}(x)>0 \\
\left.\mathrm{~B}_{\mathrm{k}}\right):\left|\nabla^{i} R\right| \leq C_{i}, 0 \leq i \leq k .
\end{gathered}
$$

These conditions can be expressed as follows:

I) The exponential map $\exp _{x}: T_{x} M \rightarrow M$ is a diffeomorphism from an open ball $B_{x}(0, r) \subset T_{x} M$ of radius $r$ around 0 in $T_{x} M$ onto an open neighborhood $U_{x, r} \subset$ $M$ of $x$ in $M$. Let $r_{x}:=\sup (r)$ be the biggest radius in $T_{x} M$ such that $\exp _{x}$ is a diffeomorphism. Then the injectivity radius is defined by $r_{i n j}:=\inf _{x \in M} r_{x}$, i.e $r_{i n j}$ is the smallest distance from $x$ where geodesics intersect. Hence,

I) $\Leftrightarrow$ there exists a ball around 0 in $\mathbb{R}^{n}$ which is domain of normal (geodesic) coordinates for all $x \in M$. 
$\left.\mathrm{B}_{\mathrm{k}}\right) \Leftrightarrow$ there exists a constant $d_{k}$ (independent of $x \in M$ ) such that $\left\|g_{i j}\right\|_{C^{k}} \leq d_{k}$ in any normal coordinate system

$\Leftrightarrow\left|D^{\alpha} g_{i j}\right| \leq c_{\alpha},|\alpha| \leq k$ in any normal coordinate system

$\Leftrightarrow\left\|\Gamma_{i j}^{m}\right\|_{C^{k-1}} \leq d_{k}^{\prime}$ in any normal coordinate system.

Examples of manifolds with bounded geometry are compact manifolds, Lie groups, homogeneous spaces, covering spaces of Riemannian manifolds, leaves of foliations of compact manifolds.

Fact: There is no topological obstruction for the existence of a complete Riemannian metric with bounded geometry of any order.

\subsection{Bounded Maps $C^{\infty, r}(M, N)$}

We consider maps which are adapted to the bounded geometry of the manifolds. Let $(M, g)$ and $(N, h)$ be open, complete Riemannian manifolds satisfying I) and $\left.\mathrm{B}_{\mathrm{k}}\right)$ and $f \in C^{\infty}(M, N)$. Then the differential $\mathrm{d} f=f_{*}=T f$ is a section of $T^{*} M \otimes f^{*} T N$. We endow $f^{*} T N$ with the induced connection $f^{*} \nabla^{h}$. Then $\nabla^{g}$ and $f^{*} \nabla^{h}$ induce connections $\nabla$ in all tensor bundles $T_{s}^{q}(M) \otimes f^{*} T_{v}^{u}(N)$. Therefore the $m^{\text {th }}$ derivative $\nabla^{m} d f$ is well defined. Assume $r \leq k$. We denote by $C^{\infty, r}(M, N)$ the set of all $f \in C^{\infty}(M, N)$ satisfying

$$
|\mathrm{d} f|_{r}:=\sum_{i=0}^{r-1} \sup _{x \in M}\left|\nabla^{i} \mathrm{~d} f\right|_{x}<\infty .
$$

Equivalently: $f \in C^{\infty, r}(M, N) \Leftrightarrow \frac{\partial^{\alpha}}{\partial x^{\alpha}} f^{\nu}$ is uniformly bounded in any normal coordinate system for all $|\alpha| \leq r, 1 \leq r \leq k$.

The topology on $C^{\infty, r}(M, N)$ :

Let $f \in C^{\infty, r}(M, N)$ and $\xi \in C^{\infty}\left(f^{*} T N\right)$. Define $g_{\xi}: M \rightarrow N$ by $g_{\xi}(x):=$ $\exp _{f(x)}(\xi(f(x)),(\equiv \exp \xi)$. Assume $r \leq k$ and define

$$
|\xi|_{r}:=\sum_{i=0}^{r} \sup _{x \in M}\left|\nabla^{i} \xi\right|_{x}<\delta_{N}<r_{i n j}(N) .
$$

Then $g_{\xi}=\exp \xi \in C^{\infty, r}(M, N)$. We define $f$ to be close to $g$ in $C^{\infty, r}(M, N)$ in the $L_{p}$-category as follows. Let $0<\delta<\frac{1}{2} r_{i n j}(N), 1<p<\infty$.

$$
\begin{gathered}
V_{\delta}:=\left\{f, g \in C^{\infty, r}(M, N) ; \exists \xi \in C^{\infty}\left(f^{*} T N\right) \text { such that } g=g_{\xi}\right\} \\
\text { and } \left.|\xi|_{p, r}:=\left(\int_{M} \sum_{i=0}^{r}\left|\nabla^{i} \xi\right|_{x}^{p} \mathrm{~d} \operatorname{vol}_{x}(g)\right)^{1 / p}<\delta\right\} .
\end{gathered}
$$


Then $\mathcal{V}:=\left\{V_{\delta}\right\}_{0<\delta<r_{i n j}(M) / 2}$ is a basis for a metrizable uniform structure on $C^{\infty, r}(M, N)$. Let $C^{p, r}(M, N)$ be the completion of $C^{\infty, r}(M, N)$ in this uniform structure. Then $C^{p, r}(M, N)$ is a $C^{k+1-r}$-Banach manifold and for $p=2$ a Hilbert manifold.

\section{Remarks:}

1) A neighborhood $\mathcal{U}_{\varepsilon}(f)$ of $f \in C^{p, r}(M, N)$ is given by:

$$
\mathcal{U}_{\varepsilon}(f)=\left\{g \in C^{p, r}(M, N) ; g=\exp \xi, \xi \in C^{\infty}\left(f^{*} T N\right),|\xi|_{p, m}<\varepsilon\right\}
$$

where $0<\varepsilon<r_{i n j}(N)$.

2) The tangent space at $f$ is given by $T_{f} C^{p, r}(M, N)=C^{p, r}\left(f^{*} T N\right)$.

3) The change of coordinates is given by $\exp _{g} \circ \exp _{f}^{-1}$, it is $C^{k-r+1}$. If $M$ is compact then $k=\infty$.

4) If $g \in \operatorname{comp}(f)$ then $T_{f} C^{p, r}(M, N) \simeq T_{g} C^{p, r}(M, N)$, otherwise not.

\subsection{The Bounded Diffeomorphism Group Diff ${ }^{p, r}(M)$}

Problem: $f$ bounded $\nRightarrow f^{-1}$ bounded, i.e. $C^{\infty, m}(M) \bigcap \operatorname{Diff}(M)$ is not a group, hence $f \in C^{\infty, m}(M) \bigcap \operatorname{Diff}(M) \nRightarrow f^{-1} \in C^{\infty, m}(M)$. We need an additional assumption to obtain a group. Let $|\lambda|_{\min }(\mathrm{d} f)$ denote the absolute value of the eigenvalues of the Jacobian of $f$ and set

$$
\operatorname{Diff}^{p, r}(M):=\left\{f \in C^{p, r}(M, M) ; f \text { bijective and }|\lambda|_{\min }(\mathrm{d} f)>0\right\}
$$

then $\operatorname{Diff}^{p, r}(M)$ is open in $C^{p, r}(M, M)$, hence a $C^{k+1-r}$ Banach manifold and we have the following

Theorem 34. (Eichhorn and Schmid [15]) Let $\left(M^{n}, g\right)$ be an open, oriented, complete Riemannian manifold satisfying $\left.\mathrm{I}), \mathrm{B}_{\infty}\right)$ and let $r>\frac{n}{p}+1$. Then $\operatorname{Diff}^{p, \infty}(M)=\lim _{\leftarrow} \operatorname{Diff}^{p, r}(M)$ is an ILB-Lie group and for $p=2$ it is an ILH-Lie group.

\subsubsection{Differentiability of the Group Operations:}

The differentiability of the group operations (composition and inversion) follow from the $\alpha$ - and $\omega$ - lemma. 
Lemma 35. ( $\boldsymbol{\alpha}$-lemma) Assume $r \leq k, r>\frac{n}{p}+1, f \in \operatorname{Diff}^{p, r}(M)$. Then the right multiplication $\alpha_{f}: \operatorname{Diff}_{0}^{p, r}(M) \rightarrow \operatorname{Diff}^{p, r}(M), \alpha_{f}(g)=g \circ f$ is of class $C^{k+1-r}$.

Lemma 36. ( $\boldsymbol{\omega}$-lemma) Let $k+1-(r+s)>s, f \in \operatorname{Diff}_{0}^{p, r+s}(M) \subset \operatorname{Diff}_{0}^{p, r}(M)$, $r>\frac{n}{p}+1$. Then the left multiplication $\omega_{f}: \operatorname{Diff}^{p, r}(M) \rightarrow \operatorname{Diff}^{p, r}(M), \omega_{f}(g)=$ $f \circ g$ is of class $C^{s}$.

Theorem 37. (Eichhorn and Schmid [15]) Let $\left(M^{n}, g\right)$ be an open, oriented, complete Riemannian manifold satisfying $\left.\mathrm{I}), \mathrm{B}_{\infty}\right)$. Let $\operatorname{Diff}_{0}^{p, \infty}(M):=$ $\lim _{\leftarrow} \operatorname{Diff}_{0}^{p, r}(M)$ with the inverse limit topology. Then

$$
\left\{\operatorname{Diff}_{0}^{p, \infty}(M), \operatorname{Diff}_{0}^{p, r}(M) ; r>\frac{n}{p}+1\right\}
$$

is an ILB-Lie group and for $p=2$ it is an ILH-Lie group.

\subsection{Volume Preserving and Symplectic Diffeomorphisms.}

We have analogous results as in the compact case for the subgroups of volume preserving and symplectic diffeomorphism. The ideas of the proofs are similar but technically much more complicated, so we just state the results (for proofs see Eichhorn and Schmid [15]). Let $\omega$ be a $C^{\infty}$-bounded non-degenerate $q$-form, $q=n$ or $q=2$, let $\operatorname{Diff}_{\omega}^{p, r}=\left\{f \in \operatorname{Diff}^{r} ; f^{*} \omega=\omega\right\}$.

Theorem 38. (Eichhorn and Schmid [15])

a) $\operatorname{Diff}_{\omega}^{p, \infty}=\lim _{\leftarrow r} \operatorname{Diff}_{\omega}^{p, r}$ is an ILH-Lie group with Lie algebra consisting of divergence free $(q=n)$, or locally Hamiltonian $(q=2)$ vector fields $\xi$ with finite Sobolev norm $|\xi|_{p, r}$ for all $r$.

b) Diff ${ }_{\omega}^{p, r}$ is an infinite dimensional Riemannian manifold, with (weak) metric

$$
g(X, Y)_{\mathrm{id}}=\int_{M}(X, Y)_{x} \mathrm{~d} \operatorname{vol}_{x}(g) .
$$

\subsection{Contact Transformations on $\dot{T}^{*} M$}

If $\left(M^{n}, g\right)$ is an open, oriented, complete Riemannian manifold satisfying I) and $\mathrm{B}_{k}$ ) then the Sasaki metric on the co-sphere bundle in $\dot{T}^{*} M$ satisfies I) and $\mathrm{B}_{k-1}$ ). Let $\theta$ be the canonical 1 -form on $T^{*} M$ and consider

$$
\operatorname{Diff}_{\theta}^{p, r}\left(\dot{T}^{*} M\right)=\left\{f \in \operatorname{Diff}^{p, r}\left(\dot{T}^{*} M\right) ; f^{*} \theta=\theta\right\} .
$$


Theorem 39. (Eichhorn and Schmid [15])

$$
\operatorname{Diff}_{\theta}^{p, \infty}\left(\dot{T}^{*} M\right)=\lim _{\infty \leftarrow r} \operatorname{Diff}_{\theta}^{p, r}\left(\dot{T}^{*} M\right)
$$

is an ILH-Lie group.

This is the space of phase functions for the Fourier integral operators!

\subsection{Pseudodifferential Operators and Fourier Integral Operators on Open Manifolds}

If $\left(M^{n}, g\right)$ is open the previous definitions of pseudodifferential operators and Fourier integral operators does not make sense. We need to adapt the class of symbols and phase functions to the bounded geometry of $M$ in order to obtain globally defined Fourier integral operators $A: C_{c}^{\infty}(M) \rightarrow \mathcal{D}^{\prime}(M)$. Then the corresponding spaces $\Psi D O$ and FIO have similar properties as in the compact case and we can use the same ideas as before to construct Lie group structures. We need the following adaptations for a Fourier integral operator

$$
A u(x)=(2 \pi)^{-n} \iint \mathrm{e}^{\mathrm{i} \varphi(x, y, \xi)} a(x, \xi) u(y) \mathrm{d} y \mathrm{~d} \xi .
$$

- Symbols: The family of local symbols $a(x, \xi)$ together with their derivatives should be uniformly bounded.

- Phase functions: The phase functions $\varphi(x, y, \xi)$ should locally generate canonical transformations in the space $\operatorname{Diff}_{\theta}^{p, r}\left(\dot{T}^{*} M\right)$.

These symbols and phase functions define the class of so called uniform pseudo differential and Fourier integral operators denoted by $\mathcal{U} \Psi D O_{m}$ and $\mathcal{U} F I O_{m}$ respectively. The details are quite technical, so we present here only the basic ideas and final result and refer to Eichhorn and Schmid [16] for details.

As in the compact case (Section 6) we get an exact sequence of groups

$$
I \rightarrow\left(\mathcal{U} \Psi D O_{0}\right)_{*} \stackrel{j}{\hookrightarrow}\left(\mathcal{U} F I O_{0}\right)_{*} \stackrel{p}{\rightarrow} \operatorname{Diff}_{\theta}^{p, r}\left(\dot{T}^{*} M\right) \rightarrow \mathrm{id} .
$$

Now we follow the same ideas as in the compact case: step 1,2...7 to construct ILH Lie groups structures on these spaces.

Theorem 40. (Eichhorn and Schmid [16])

$$
\begin{aligned}
& \mathcal{U} \Psi D O=\lim _{\infty \leftarrow s} \mathcal{U} \Psi D O^{s} \text { is an ILH Lie group } \\
& \mathcal{U} F I O=\lim _{\infty \leftarrow t} \mathcal{U} F I O^{t} \text { is an ILH Lie group }
\end{aligned}
$$


- the multiplication $\mu: \mathcal{U} F I O^{t+r} \times \mathcal{U F I O}{ }^{t} \rightarrow \mathcal{U} F I O^{t} \mu(A, B)=A B$ is $C^{k}$ differentiable, $k=\min (r, t)$

- the inversion $\nu: \mathcal{U} F I O^{t+r} \rightarrow \mathcal{U} F I O^{t} ; \nu(A)=A^{-1}$ is $C^{k}$ differentiable, $k=\min (r, t)$

- the right multiplications $R_{A}: \mathcal{U F I O}{ }^{t} \rightarrow \mathcal{U F I O}{ }^{t}: R_{A}(B)=B A$ are $C^{t}$ differentiable, for any $A \in \mathcal{U} F I O^{t}$

- the left multiplications $L_{A}: \mathcal{U} F I O^{t} \rightarrow \mathcal{U F I O}{ }^{t}: L_{A}(B)=A B$ are $C^{0}$ (continuous), for any $A \in \mathcal{U} F I O^{t}$.

\section{Applications to Fluid Dynamics and Quantization}

We briefly discuss some applications to fluid dynamics, the periodic and nonperiodic KdV equations, the classical, topological and non-homogeneous Euler equations, as well as quantization. Some of this work is still in progress.

\subsection{The KdV Equation and the Group of Fourier Integral Operators}

The Korteweg deVries (KdV) equation $u_{t}=6 u u_{x}-u_{x x x}$ is an infinite dimensional Hamiltonian system with respect to the Poisson bracket

$$
\{F, G\}(u)=\int \frac{\delta F}{\delta u} \partial_{x} \frac{\delta G}{\delta u} \mathrm{~d} x
$$

and Hamiltonian

$$
H(u)=\int\left(u^{3}+\frac{1}{2} u_{x}^{2}\right) \mathrm{d} x
$$

which means that a function $u$ satisfies Hamiltons equations $u_{t}=\{u, H\}$ if and only if $u$ is a solution of the KdV equation. This was shown by Gardner and Kruskal in 1971.

The question we asked is: Where does this Poisson bracket (124) and this Hamiltonian (125) come from? Is there a natural space on which this Hamiltonian system lives? And the answer is the following:

Theorem 41. (Adams, Ratiu and Schmid [1])

A) The Poisson bracket (124) is the natural Lie-Poisson bracket on the coadjoint orbit of the Lie group of invertible Fourier integral operators $\mathcal{G}=F I O_{*}$ through the Schrödinger operator.

B) The Kostant-Symes theorem applied to a splitting of the Lie algebra of FIO*, 
the space of pseudodifferential operators $\mathfrak{g}=\Psi D O$ gives the complete integrability of KdV, i.e. the Gelfand-Dikii family of commuting integrals, including the Hamlitonian (125).

We can only outline the ideas of the proves and refer to [1] for details.

The Kostant-Symes Theorem [23],[40] states the following: Suppose we have a vector space decomposition of a Lie algebra $\mathfrak{g}$ into a direct sum of two subalgebras, $\mathfrak{g}=\mathfrak{h} \oplus \mathfrak{k}$. This gives the corresponding decomposition of the dual $\mathfrak{g}^{*}=\mathfrak{k}^{\perp} \oplus \mathfrak{h}^{\perp}$ which allows us to identify the duals $\mathfrak{h}^{*} \cong \mathfrak{k}^{\perp}$ and $\mathfrak{k}^{*} \cong \mathfrak{h}^{\perp}$. We obtain functions in involution as follows: Let $F, H: \mathfrak{g}^{*} \rightarrow \mathbb{R}$ be two functions that are constant on coadjoint orbits of $G$ in $\mathfrak{g}^{*}$. Then for $A \in \mathfrak{h}^{*},\left\{F_{A}, H_{A}\right\}=0$, where $F_{A}$ and $H_{A}$ are the restriction of $F$ and $H$ to the coadjoint orbit of $A$ in $\mathfrak{h}^{*}$. For the $\mathrm{KdV}$ equation the Lie group is $G=F I O_{*}\left(S^{1}\right)$ with Lie algebra $\mathfrak{g}=$ $\Psi D O\left(S^{1}\right)$, where $M=S^{1}$ is the unit circle. Then each pseudodifferential operator $P \in \Psi D O_{m}\left(S^{1}\right)$ has a total symbol of the form $p(x, \xi)=\sum_{-\infty<j \leq m} p_{j}(x) \xi^{j}$. The Lie algebra $\mathfrak{g}=\Psi D O$ decomposes into the two subalgebras $\mathfrak{h}=\Psi D O_{-}=$ $\cup_{m<0} \Psi D O_{m}$ and $\mathfrak{k}=\Psi D O_{+}=\cup_{m \geq 0} \Psi D O_{m}$, i.e. $\mathfrak{g}=\mathfrak{h} \oplus \mathfrak{k}$ becomes $\Psi D O=$ $\Psi D O_{-} \oplus \Psi D O_{+}$.

We have an inner product $\langle P, Q\rangle:=\operatorname{trace}(P \cdot Q)$ where the trace is defined by $\operatorname{trace}(P):=\int p_{-1}(x) \mathrm{d} x$. With this we identifies $\Psi D O^{*} \simeq \Psi D O$ and $\left(\Psi D O_{-}\right)^{*} \simeq \Psi D O_{+}$. So for $\mathfrak{g}=\mathfrak{h} \oplus \mathfrak{k}$ we get $\mathfrak{g}^{*}=\mathfrak{k}^{\perp} \oplus \mathfrak{h}^{\perp}$, i.e

$$
\mathfrak{g}^{*}=\Psi D O^{*} \simeq \Psi D O_{+}^{\perp} \oplus \Psi D O_{-}^{\perp} \simeq \Psi D O_{-}^{*} \oplus \Psi D O_{-}^{\perp} \simeq \Psi D O_{+} \oplus \Psi D O_{-}^{\perp} .
$$

The Lie-Poisson bracket on $\mathfrak{h}^{*}=\Psi D O_{-}^{*} \simeq \Psi D O_{+}$at $A \in \Psi D O_{+}$becomes

$$
\{F, H\}(A)=\left\langle A,\left[\frac{\delta F}{\delta A}, \frac{\delta H}{\delta A}\right]\right\rangle=\int\left(A \circ\left[\frac{\delta F}{\delta A}, \frac{\delta H}{\delta A}\right]\right)_{-1} \mathrm{~d} x
$$

where $(\ldots)_{-1}$ means taking the order $(-1)$ part of the symbol.

The Lie-Poisson evolution equations $\dot{F}=\{F, H\}$ for any function $F$ on $\Psi D O_{-}^{*}$ are equivalent to

$$
\dot{A}=X_{H}(A)=\operatorname{ad}_{\frac{\delta H}{\delta A}}^{*}(A)=\left[\frac{\delta H}{\delta A}, A\right]_{+}
$$

on $\Psi D O_{-}^{*} \simeq \Psi D O_{+}$, where $[\ldots]_{+}$means taking only the part in $\Psi D O_{+}$. For the Schrödinger operator $A \in \Psi D O_{+}$with total symbol given by $a(x, \xi)=a(x)+\xi^{2}$ the Lie-Poisson bracket of two functions $F, G: \Psi D O^{*} \rightarrow \mathbb{R}$ at $A$ becomes

$$
\{F, G\}=\int \frac{\delta F}{\delta a} \partial_{x} \frac{\delta G}{\delta a} \mathrm{~d} x \text {, which is the Gardner bracket (124). }
$$


For the functionals $H_{k}(A)=\operatorname{trace}\left(A^{k}\right)=\int\left(A^{k}\right)_{-1} \mathrm{~d} x, k \in \mathbb{N}$ we have $\frac{\delta H_{k}}{\delta A}=k A^{k-1}$, hence $\left[A, \frac{\delta H_{k}}{\delta A}\right]=\left[A, k A^{k-1}\right]=0$.

Thus $H_{k}$ are constant on coadjoint orbits. By the Kostant-Symes theorem, restricting the $H_{k}$ to $\Psi D O_{-}^{*} \simeq \Psi D O_{+}$gives the Gelfand-Dikii family of commuting integrals for $\mathrm{KdV}$.

We get the following integrals, for example: $H_{0}=\int a \mathrm{~d} x, H_{1}=\int \frac{1}{2} a^{2} \mathrm{~d} x$,

$$
\begin{aligned}
H_{2} & =\int\left(a^{3}+\frac{1}{2} a_{x}^{2}\right) \mathrm{d} x \equiv H, \text { which is the Gardner Hamiltonian (125) } \\
H_{3} & =\int\left(\frac{5}{8} a^{4}+\frac{5}{4} a a_{x}^{2}+\frac{1}{8} a_{x x}^{2}\right) \mathrm{d} x \text { etc. }
\end{aligned}
$$

\subsection{The KdV Equation and the Lie Group $\mathcal{U} F I O_{*}$}

We showed above that the periodic KdV equation (which describes shallow water waves) is a Hamiltonian system on a coadjoint orbit of the Lie group of Fourier integral operators on the compact manifold $M=S^{1}$ the circle. Having done all the work for open manifolds we proved the similar result for the non periodic $\mathrm{KdV}$ equation on the real line $M=\mathbb{R}$.

Theorem 42. (Eichhorn and Schmid [17]) The non-periodic KdV equation on the real line is a Hamiltonian system with respect to the Lie-Poisson bracket on the coadjoint orbit through the Schrödinger operator of the infinite dimensional Lie group of invertible Fourier integral operators $\mathcal{G}=\mathcal{U} F I O_{*}(\mathbb{R})$.

\subsection{Hydrodynamics and the Diffeomorphism Group $\operatorname{Diff}_{\mu}^{\infty, r}(M)$ \\ 1. The Euler Equations and $\operatorname{Diff}_{\mu}^{\infty, r}(M)$}

The topological Euler equations are given by

$$
E_{t o p}\left\{\begin{aligned}
\frac{\partial u}{\partial t}+\nabla_{u(t)} u(t) & =\operatorname{grad} p \\
\operatorname{div}_{\mu} u(t) & =0
\end{aligned}\right.
$$

We call these equations the topological Euler equations because $\mu$ is a fixed volume form on $\left(M^{n}, g\right)$, not necessarily the phase space volume given by the 
Riemannian metric $g$. So we have two different volume forms on $M$. The covariant derivative $\nabla=\nabla^{g}$ is taken with respect to the Riemannian metric $g$, whereas the divergence $\operatorname{div}=\operatorname{div}_{\mu}$, defined by $L_{X} \mu=\left(\operatorname{div}_{\mu} X\right) \mu$, is the divergence with respect to $\mu$. The vector field $u=u(x, t)$ is a time dependent $C^{1}$ vector field on $\left(M^{n}, g\right)$. Then $u(t)$ defines a 1-parameter family of diffeomorphisms $f_{t}$ defined by

$$
\left.\frac{\mathrm{d} f_{s}}{\mathrm{~d} s}\right|_{s=t}=u(t) \circ f_{t}
$$

We have the analogoues result as discussed in Section 4.1, Theorem 11 ( EbinMarsden) for non-compact manifolds.

Theorem 43. (Eichhorn and Schmid [15]) Assume $\left(M^{n}, g\right)$ is an open Riemannian manifold satisfying I) and $\left.\mathrm{B}_{\mathrm{k}}\right)$. Then $u(t)$ satisfies the topological Euler equations $E_{\text {top }}$ iff $\left\{f_{t}\right\}_{t}$ is a geodesic in $\operatorname{Diff}_{\mu}^{\infty, r}(M)$.

The classical Euler equations for an incompressible, homogeneous fluid without viscosity are a special case with $\mu=\mathrm{d} v o l_{x}(g)$ the volume of the Riemannian metric $g$ and $\nabla=\nabla^{g}, \operatorname{div}=\operatorname{div}_{\mathrm{d}{ }_{0} l_{x}(g)}$.

\section{The Non-Homogeneous Euler Equations}

The non-homogeneous Euler equations with a mass density $\rho(x, t)>0$ are given by

$$
E_{N H}\left\{\begin{aligned}
\frac{\partial u}{\partial t}+\nabla_{u(t)} u(t) & =\frac{1}{\rho} \operatorname{grad} p \\
\frac{\partial \rho}{\partial t}+\operatorname{grad} \rho \cdot u & =0 \\
\operatorname{div}_{\mu} u(t) & =0 .
\end{aligned}\right.
$$

If $\rho=$ constant these are the classical homogeneous Euler equations. For $\mu=$ $\mu(g)$ the corresponding equations on $\operatorname{Diff}_{\mu}^{s}(M)$ are not right invariant, i.e. they are not derivable from Arnold's method as above. But if we take $\tilde{\mu}=\rho_{o} \mu(g)$ as volume form, then we have the following

Theorem 44. (Eichhorn and Schmid [17]) $u(t)$ is a solution of $E_{N H} \Leftrightarrow\left(f_{t}\right)_{t}$ the flow of $u(t)$ is a geodesic on $\operatorname{Diff}_{\tilde{\mu}}^{s}(M)$ and $\rho(x, t)=\rho_{o}\left(f_{t}^{-1}(x)\right)$, where the volume form is $\tilde{\mu}=\rho_{o} \mu(g)$.

Proof: One needs a generalization of the Hodge decomposition theorem with densities for open manifolds (work in progress). 


\section{4. $\Psi D O$ and Quantization}

Another interpretation of the exact sequences of Lie groups (99) and their Lie algebras (100) discussed in Section 6 leads to quantization

$$
\begin{aligned}
& I \longrightarrow\left(\Psi D O_{0}\right) * \stackrel{j}{\hookrightarrow}\left(F I O_{0}\right) * \stackrel{p}{\longrightarrow} \operatorname{Diff}_{\theta}^{s}\left(\dot{T}^{*} M\right) \longrightarrow e \\
& 0 \longrightarrow \Psi D O_{0} \stackrel{j}{\hookrightarrow} \Psi D O_{1} \stackrel{\pi}{\longrightarrow} C_{+1}^{\infty}\left(\dot{T}^{*} M\right) \longrightarrow 0 .
\end{aligned}
$$

For $P \in \Psi D O_{1}$ its principal symbol $\pi(P): \dot{T}^{*} M \rightarrow \mathbb{R}$ is a smooth function, homogeneous of degree +1 . Hence $\pi(P) \in C_{+1}^{\infty}\left(\dot{T}^{*} M\right)$. Moreover $\pi$ is a surjective Lie algebra homomorphism

$$
\pi([P, Q])=\frac{1}{\mathrm{i}}\{\pi(P), \pi(Q)\} .
$$

That means the commutator bracket of the operators corresponds to the Poisson brackets of their principal symbols, which means quantization of $C_{+1}^{\infty}\left(\dot{T}^{*} M\right)$ via $\Psi D O_{1}$ !

Consider the Lie subalgebra $D O_{1} \subset \Psi D O_{1}$ of all differential operators of order 1 which is isomorphic to the space of all smooth vector filed on $M$, i.e. $D O_{1} \cong$ $\mathfrak{X}^{\infty}(M)$. We consider a vector field $X$ on $M$ as a pseudodifferential operator of order 1. Locally we have $X=\sum X^{j}(x) \frac{\partial}{\partial x_{j}}$, hence its principal symbol is given by $\pi(X)=\sum X^{j} \xi_{j}$ and we write $X$ as pseudodifferential operator

$$
X u(x)=(2 \pi)^{-n} \iint \mathrm{e}^{\mathrm{i}(x-y) \cdot \xi} \pi(X) u(y) \mathrm{d} y \mathrm{~d} \xi .
$$

Consider the Lie subalgebra $L\left(T^{*} M\right) \subset C_{+1}^{\infty}\left(\dot{T}^{*} M\right)$ of all smooth functions on $T^{*} M$ linear on each fiber $T_{x}^{*} M$. Locally $f \in L\left(T^{*} M\right)$ is of the form $f(x, \xi)=$ $\sum f^{j}(x) \xi_{j}$.

Theorem 45. (Schmid [37]) The symbol map $\pi$ induces a Lie algebra isomorphism $\pi: \mathfrak{X}^{\infty}(M) \rightarrow L\left(T^{*} M\right): \pi(X) \cdot \alpha_{x}=\mathrm{i} \alpha_{x} \cdot X(x), x \in M, \alpha_{x} \in T_{x}^{*} M$

$$
\pi([X, Y])=\frac{1}{\mathrm{i}}\{\pi(X), \pi(Y)\} .
$$

This means quantization of $L\left(T^{*} M\right)$ via $\mathfrak{X}^{\infty}(M)$.

In general, by Egorov's theorem we have the following: For any $f, g \in C^{\infty}\left(T^{*} M\right)$ define $P, Q \in \Psi D O$ such that $P$ has principal symbol $f$ and $Q$ has principal symbol $g$. Then the principal symbol of $[P, Q]$ if $\{f, g\}$.

References: Because of space limitations we give only a short, incomplete list of references, but more detailed references can be found in the papers listed below. 


\section{References}

[1] Adams M., Ratiu T. and Schmid, R., The Lie Group Structure of Diffeomorphism Groups and Invertible Fourier Integral Operators, with Applications, MSRI Publications, vol. 4, V. Kac (Ed), (1985) pp. 1-69.

[2] Adams M., Ratiu, T. and Schmid R., A Lie Group Structure for Pseudodifferential Operators, Math. Ann. 273 (1986) 529-551.

[3] Adams M., Ratiu T. and Schmid R., A Lie Group Structure for Fourier Integral Operators, Math. Ann. 276 (1986) 19-41.

[4] Arnold V., One-Dimensional Cohomology of Lie Algebras of Nondivergent Vector Fields and Rotation Numbers of Dynamical Systems, Funct. Anal. Appl. 3 (1969) 319-321.

[5] Banyaga A., The Structure of Classical Diffeomorphism Groups, Kluwer., Dortrecht, 1997.

[6] Becci C., Rouet A. and Stora, R., Renormalization of Gauge Groups, Ann. Physics 98 (1976) 287-321.

[7] Booss B. and Bleecker, D., Topology and Analysis, Springer, New York, 1985.

[8] Bourbaki N., Lie Groups and Lie Algebras, Hermann, Paris, 1975.

[9] Calabi E., On the Group of Automorphisms of a Symplectic Manifold, In: Problems in Analysis, Symposium ih Honor of S. Bochner, Princeton Univ. Press, Princeton, 1970, pp.,1-26.

[10] Chevalley C., Theory of Lie Groups, Princeton Univ. Press, Princeton, 1946.

[11] Chevalley C., Eilenberg S., Cohomology Theory of Lie Groups and Lie Algebras, Trans. Amer. Math. Soc. 63 (1948) 85-124.

[12] Deligne P. et al. (Eds), Quantum Field Theory and Strings: A Course for Mathematicians, Vols. 1 and 2, Amer. Math. Soc., Providence, 1999.

[13] Ebin D. and Marsden J., Groups of Diffeomorphisms and the Motion of Incompressible Fluid, Ann. of Math. 92 (1970) 102-163.

[14] Eichhorn J., The Manifold Structure of Maps between Open Manifolds, Ann. Global Anal. Geom. 11 (1993) 253-300.

[15] Eichhorn J. and Schmid, R., Form Preserving Diffeomorphism Groups on Open Manifolds, Ann. Global Anal. Geom. 14 (1996) 147-176.

[16] Eichhorn J. and Schmid R., Lie Groups of Fourier Integral Operators on Open Manifolds, Comm. Analysis and Geometry 9 (2001) 983-1040.

[17] Eichhorn J. and Schmid R., Applications of the Lie Group of Fourier Integral Operators to Fluid Dynamics, Preprint, 2003. 
[18] Goldin G., The Diffeomorphism Group Approach to Nonlinear Quantum Systems. Int. J. Mod. Physics B 6 (1992) 1905-1916.

[19] Goldin G., Menikoff R. and Sharp D., Diffeomorphism Groups, Gauge Groups, and Quantum Theory. Phys. Rev. Lett. 51 (1983) 2246-2249.

[20] Hamilton R., The Inverse Function Theorem of Nash and Moser, Bull. Amer. Math. Soc. 7 (1982) 65-222.

[21] Keller H., Differential Calculus in Locally Concex Spaces, Lecture Notes in Math. Vol. 417, Springer, Berlin, 1974.

[22] Kostant B., Quantization and Unitary Representations, Lectures in Modern Analysis and Applications III, Lecture Notes in Math. Vol. 170 (1970) 87208.

[23] Kostant B., The Solution to a Generalized Toda Lattice and Representation Theory, Adv. Math. 34 (1979) 195-338.

[24] Lang S., Introduction to Differentiable Manifolds, John Wiley, New York, 1962.

[25] Marsden J., Ratiu T., Schmid R., Spencer R. and Weinstein A., Hamiltonian Systems with Symmetries, Coadjoint Orbits and Plasma Physics, Atti Accad. Sci. Torino Cl. Sci. Fis. Mat. Natur. 117 (1983) 289-340.

[26] Moser J., A Rapidly Convergent Iteration Method and Non-linear Differential Equations, Ann. Scuola Norm. Sup. Pisa Cl. Sci. 20 (1966) 499-535.

[27] Nash J., The Embedding Problem for Riemannian Manifolds, Ann. of Math. 63 (1956) 20-63.

[28] Omori H., Infinite Dimensional Lie Transformation Groups, Lecture Notes in Math. Vol. 427, Berlin, 1974.

[29] Omori H., Infinite-Dimensional Lie Groups, Transl. Math. Monographs Vol. 158, Amer. Math. Soc., Providence, 1996.

[30] Palais R., Foundations of Global Nonlinear Analysis, Addison-Wesley, Reading, 1968.

[31] Pressley A. and Segal G., Loop Groups, Oxford Univ. Press, Oxford, 1986.

[32] Ratiu T. and Schmid R., The Differential Structure of Three Remarkable Diffeomorphism Groups, Math. Z. 177 (1981) 81-100.

[33] Schmid R., Die Symplektomorphismen-Gruppe als Frechet Lie Gruppe, PhD Thesis, Juris Druck + Verlag, Zuerich, 1978.

[34] Schmid R., The Inverse Function Theorem of Nash and Moser for the $\Gamma$ Differentiability, Abh. Akad. Wiss. Berlin 1984-2N (1984) 201-206.

[35] Schmid R., Infinite Dimensional Hamiltonian Systems, Monographs and Textbooks in Physical Sciences, Biblionopolis, Napoli, 1987. 
[36] Schmid R., The Geometry of BRS Transformations, Illinois J. Math., 34 (1990) 87-97.

[37] Schmid R., Diffeomorphism Groups, Pseudodifferential Operators and rMatrices, In: Global Differential Geometry, D. Ferrus et al (Eds), Lecture Notes in Math. Vol. 1481 (1991) 258-263.

[38] Schmid R., Local Cohomology in Gauge Theories, BRST Transformations and Anomalies, Differential Geom. Appl. 4 (1994) 107-116.

[39] Schmid R., g-Symplectic Orbits and a Solution of the BRST Consistency Condition, In: Geometry, Integrability and Quantization IV, I. Mladenov and G. Naber (Eds), Coral Press, Sofia 2003, 284-295.

[40] Symes W., Hamiltonian Group Actions and Integrable Systems, Physica D 1 (1980) 339-374.

[41] Tyutin I., Gauge Invariance in Field Theory and Statistical Mechanics, Lebedev Inst. Preprint 39, 1976, unpublished.

[42] Varadarajan V., Lie Groups, Lie Algebras, and their Representations, Prentice-Hall, Inc., Englewood Cliffs, 1974.

[43] Weinstein A., Symplectic Manifolds and their Lagrangian Submanifolds, Adv. Math. 6 (1971) 329-346.

Department of Mathematics

EMORY University

Atlanta, GA 30032

USA

e-mail: rudolf@mathcs.emory.edu

web: www.math.emory.edu/ rudolf 